\title{
Independence of time and position for a random walk
}

\section{Christophe Ackermann, Gérard Lorang and Bernard Roynette}

\begin{abstract}
Given a real-valued random variable $X$ whose Laplace transform is analytic in a neighbourhood of 0 , we consider a random walk $\left(S_{n}, n \geq 0\right)$, starting from the origin and with increments distributed as $X$. We investigate the class of stopping times $T$ which are independent of $S_{T}$ and standard, i.e. $\left(S_{n \wedge T}, n \geq 0\right)$ is uniformly integrable. The underlying filtration $\left(\mathcal{F}_{n}, n \geq 0\right)$ is not supposed to be natural. Our research has been deeply inspired by [7], where the analogous problem is studied, but not yet solved, for the Brownian motion. Likewise, the classification of all possible distributions for $S_{T}$ remains an open problem in the discrete setting, even though we manage to identify the solutions in the special case where $T$ is a stopping time in the natural filtration of a Bernoulli random walk and $\min T \leq 5$. Some examples illustrate our general theorems, in particular the first time where $\left|S_{n}\right|$ (resp. the age of the walk or Pitman's process) reaches a given level $a \in \mathbb{N}^{*}$. Finally, we are concerned with a related problem in two dimensions. Namely, given two independent random walks $\left(S_{n}^{\prime}, n \geq 0\right)$ and $\left(S_{n}^{\prime \prime}, n \geq 0\right)$ with the same incremental distribution, we search for stopping times $T$ such that $S_{T}^{\prime}$ and $S_{T}^{\prime \prime}$ are independent.
\end{abstract}

\section{Introduction}

B. de Meyer, B. Roynette, P. Vallois and M. Yor investigate the following problem in [7]: given a linear Brownian motion $\left(B_{t}, t \geq 0\right)$ starting from 0 and adapted to a filtration $\left(\mathcal{F}_{t}, t \geq 0\right)$, characterize the $\left(\mathcal{F}_{t}\right)$-stopping times $T$ such that (i) $\left(B_{t \wedge T}, t \geq 0\right)$ is uniformly integrable ( $T$ is then called " $B$-standard") and (ii) $T$ and $B_{T}$ are independent. This question is strongly

2000 Mathematics Subject Classification: 60G50, 60G40, 60G42, 60E10, 60J10, 60C05. Keywords: Independence, random walk, stopping time, Wald's identity, Khinchine's inequalities, Pitman's process, age process. 
related to the celebrated "Skorokhod problem" for the space-time process $\left(\left(t, B_{t}\right), t \geq 0\right)$, which consists in finding a stopping time $T$ such that $\left(T, B_{T}\right)$ follows a given distribution $\mathcal{L}$ on $\mathbb{R}_{+} \times \mathbb{R}$. The characterization of all possible stopping distributions $\mathcal{L}$ turns out to be difficult, although a necessary and sufficient condition, due to the works of Bourekh [2], Rost [11], Falkner and Fitzsimmons [3], is known. Hence it is natural to introduce the additional "simplifying" conditions (i) and (ii) above. Nevertheless, even in that case a simple description of the stopping distributions (as obtained e.g. in [12] for the two-dimensional process $\left(\left(L_{t}, B_{t}\right), t \geq 0\right)$, where $\left(L_{t}, t \geq 0\right)$ denotes the local time at 0 of the Brownian motion) remains an open problem.

In this paper, we are concerned with the discrete version of the above problem. More precisely, let $X$ be a real-valued random variable whose Laplace transform $\varphi(z)=\mathbb{E}(\exp z X)$ is analytic in some neighbourhood of 0 . This implies in particular that $X$ has finite moments of any order. Let $S=\left(S_{n}, n \geq 0\right)$ be a random walk starting from the origin, with increments distributed as $X$. We assume that $S$ is adapted to a filtration $\left(\mathcal{F}_{n}, n \geq 0\right)$ which is not necessarily the natural filtration (cf. (1.1)).

In Section 1, we investigate the class of $\left(\mathcal{F}_{n}\right)$-stopping times $T$ such that

$$
T \text { is } S \text {-standard and } T \Perp S_{T} \text {. }
$$

Here the label " $S$-standard" has the same meaning as in the case of Brownian motion, whether $X$ is centered or not (cf. definition 1.1). Theorem 1.1 gives some properties of stopping times solving (IP). First, we prove that the Laplace transform $\psi_{T}(z)=\mathbb{E}\left(\exp z S_{T}\right)$ is well-defined and holomorphic in the strip of regularity of $\varphi$. Then we establish the following relation, referred to as Wald's identity:

$$
|\varphi(z)| \geq 1 \Rightarrow \mathbb{E}\left(\exp z S_{T}\right) \mathbb{E}\left(\frac{1}{\varphi(z)^{T}}\right)=1 .
$$

Denoting by $g_{T}(r)=\mathbb{E}\left(r^{T}\right)$ the generating function of $T$, Wald's identity reads:

$$
|\varphi(z)| \geq 1 \quad \Rightarrow \quad \psi_{T}(z) g_{T}\left(\frac{1}{\varphi(z)}\right)=1 .
$$

The consequences of this equality are manifold. First of all, it shows that the law of $T$ uniquely determines that of $S_{T}$, and conversely. Moreover, it implies that the generating function of $T$ is analytic at 1 , whence $T$ has finite moments of any order. Under the stronger assumption that $g_{T}$ is an entire function and with some technical constraints on $\varphi$, we obtain that $T$ is necessarily constant. This is the object of theorem 1.2. Finally, when $\varphi$ is entire, Wald's identity permits us to relate the order and the type of $\psi_{T}$ 
to those of $\varphi$ (cf. proposition 1.1). We conclude Section 1 by improving Khinchine's inequalities for $S_{T}$, in the case where $X$ is centered and belongs to Newman's class $(\mathcal{L})$. The reader is referred to proposition 1.3 for precise statements.

Section 2 is entirely devoted to examples of stopping times solving (IP). We begin by proving a few general results when $S$ is a Bernoulli random walk, that is, when $\mathbb{P}(X=1)=p=1-\mathbb{P}(X=-1)$. In the symmetric case $(p=1 / 2)$, a direct application of Wald's identity implies that, for each $T$ satisfying (IP), there exists a polynomial $\tilde{P}_{m}$ of degree $m=\min T$ which uniquely determines the joint law of $\left(S_{T}, T\right)$ through the relations $\psi_{T}(z)=\tilde{P}_{m}(\operatorname{ch} z)$ and $g_{T}(r)=1 / \tilde{P}_{m}(1 / r)$ (cf. lemma 2.2). If in addition the filtration $\left(\mathcal{F}_{n}, n \geq 0\right)$ is assumed to be natural, it will be shown that the same $T$ also solves (IP) in the general case $0<p<1$ (cf. proposition 2.1). We study a few examples in detail, in particular the first time where $\left|S_{n}\right|$, respectively Pitman's process reaches a given level $a \in \mathbb{N}^{*}$. The most interesting example is certainly provided by the first time the age process reaches $a$; here the independence of $T$ and $S_{T}$ is realized for any distribution of $X$ (cf. proposition 2.4).

We return to the symmetric Bernoulli random walk in Section 3. Here, every probability distribution of some $S_{T}$, where $T$ is a stopping time in the natural filtration of the walk and satisfies (IP), will be called a "stopping distribution". By elementary combinatorial arguments, we obtain an algorithm which characterizes the stopping distributions according to the maximal element of their support (which is also the minimum of the corresponding stopping time) (cf. proposition 3.1 and theorem 3.1). Let us stress the fact that this algorithm is of little practical interest because infinitely many steps are necessary to check that a given distribution is actually a stopping distribution. To each stopping distribution, say $\mathcal{L}\left(S_{T}\right)$, we associate its "generating polynomial" (which, roughly speaking, is nothing else but the polynomial $\tilde{P}_{m}$ above, $m$ being the minimum of the corresponding stopping time $T$ ) and we are able to express the law of $T$ in terms of the roots of the generating polynomial (cf. proposition 3.2). Finally, we manage to identify all stopping distributions for $m \leq 5$. The general classification of stopping distributions remains an open problem.

In Section 4, we turn to a related problem in two dimensions. Given two independent symmetric Bernoulli random walks $\left(S_{n}^{\prime}, n \geq 0\right)$ and $\left(S_{n}^{\prime \prime}, n \geq 0\right)$, we investigate the class of stopping times $T$ such that $S_{T}^{\prime}$ and $S_{T}^{\prime \prime}$ are independent. We prove that when $T$ is not too large (cf. theorem 4.1 for details), $S_{T}^{\prime}$ and $S_{T}^{\prime \prime}$ are both distributed as $S_{N}$, for some integer $N$. In the appendix, we generalize this result to more general increments. 


\section{General theorems}

Let $\left(X, X_{i}, i \geq 1\right)$ be a sequence of i.i.d. real-valued random variables defined on some filtered probability space $\left(\Omega, \mathcal{F},\left(\mathcal{F}_{n}, n \geq 0\right), \mathbb{P}\right)$, such that

$$
\left\{\begin{array}{l}
\sigma\left(X_{1}, X_{2}, \ldots, X_{n}\right) \subset \mathcal{F}_{n}, n \geq 1 \\
\mathcal{F}_{n} \Perp \sigma\left(X_{n+1}, X_{n+2}, \ldots\right), n \geq 0 .
\end{array}\right.
$$

The Laplace transform $\varphi(z)=\mathbb{E}(\exp z X)$ is assumed to be analytic in a neighbourhood of 0 . Let $\mathcal{B}_{\varphi}$ denote the strip of regularity of $\varphi$, i.e.

$$
\mathcal{B}_{\varphi}=\{z \in \mathbb{C}, \varphi(\Re e z)<+\infty\}=\{z \in \mathbb{C},-\alpha<\Re e z<\beta\} .
$$

Throughout this work, it will be assumed that $0<\alpha, \beta \leq \infty$. This implies in particular that $X$ has finite moments of any order. The trivial case $X=0$ a.s. will always be excluded.

We define a random walk by $S_{0}=0$ and $S_{n}=X_{1}+X_{2}+\ldots+X_{n}, n \geq 1$. Let us recall that the centered walk $\tilde{S}=\left(S_{n}-n \mu, n \geq 0\right)$, where $\mu=\mathbb{E} X$, is a martingale with respect to the filtration $\left(\mathcal{F}_{n}, n \geq 0\right)$. When $\mu \geq 0$ (resp. $\mu \leq 0),\left(S_{n}, n \geq 0\right)$ is a submartingale (resp. a supermartingale) with respect to this filtration.

Definition 1.1 A stopping time $T$ in the filtration $\left(\mathcal{F}_{n}, n \geq 0\right)$ is said to be $S$-standard if $T$ is a.s. finite and if the stopped process $S^{T}=\left(S_{n \wedge T}, n \geq 0\right)$ is uniformly integrable.

The prefix "S-" will be omitted whenever no confusion is possible.

Lemma 1.1 If $\mathbb{E} T<+\infty$, then $T$ is standard.

Proof. If $\mathbb{E} T<+\infty$, then $\tilde{S}_{T}$ is a uniformly integrable martingale. Indeed, denoting $\sigma^{2}=\operatorname{Var} X$, it is easy to check that $\left(\tilde{S}_{n \wedge T}-(n \wedge T) \sigma^{2}, n \geq 0\right)$ is a martingale, which implies that $\mathbb{E}\left(\tilde{S}_{n \wedge T}^{2}\right)=\sigma^{2} \mathbb{E}(n \wedge T)$. Hence, the process $\tilde{S}^{T}$ is bounded in $L^{2}$ and therefore u.i. As a result, $S^{T}$ is the sum of two u.i. processes and thus is also u.i.

Remark 1.1 Retain from this proof that if $\mathbb{E} T<+\infty$, then $\tilde{S}_{T}$ is u.i.

\subsection{Independence of $T$ and $S_{T}$. Wald's identity and consequences}

For any a.s. finite $\left(\mathcal{F}_{n}\right)$-stopping time $T$, we will adopt the notation $\psi_{T}(\lambda)=$ $\mathbb{E}\left(\exp \lambda S_{T}\right)$ throughout. The following theorem gives some necessary properties of stopping times which solve the independence problem (IP) stated in the introduction. 
Theorem 1.1 Let $T$ be a $\left(\mathcal{F}_{n}\right)$-stopping time satisfying (IP). Then:

a) $\psi_{T}$ is holomorphic in the strip of regularity of $\varphi$, that is:

$$
\forall \lambda \in]-\alpha, \beta\left[, \quad \psi_{T}(\lambda)=\mathbb{E}\left(\exp \lambda S_{T}\right)<+\infty .\right.
$$

b) Wald's identity holds for all $z \in \mathbb{C}$ such that $|\varphi(z)| \geq 1$, i.e.:

$$
|\varphi(z)| \geq 1 \Rightarrow \mathbb{E}\left(\exp z S_{T}\right) \mathbb{E}\left(\frac{1}{\varphi(z)^{T}}\right)=1 .
$$

c) If $|\varphi(z)| \geq 1$ then $\left|\psi_{T}(z)\right| \geq 1$.

d) $g_{T}(r):=\mathbb{E}\left(r^{T}\right)<+\infty$, for some $r>1$, i.e. the radius of convergence of the generating function of $T$ is $>1$.

e) Setting $\mu=\mathbb{E} X$ and $\sigma^{2}=\operatorname{Var} X$, we have

$$
\mathbb{E} S_{T}=\mu \mathbb{E} T \text { and } \operatorname{Var} S_{T}=\sigma^{2} \mathbb{E} T-\mu^{2} \operatorname{Var} T .
$$

Remark 1.2 (i) By a), if $\varphi$ is an entire function, the same is true for $\psi_{T}$. (ii) If $\mu>0$ (resp. $\mu<0$ ) then (1.3) holds for all nonnegative (resp. nonpositive) real numbers. If $X$ is centered, (1.3) holds for every $z \in \mathbb{R}$.

(iii) According to d), $T$ has finite moments of any order. In particular, $T$ is of finite expectation.

(iv) Wald's identity shows that the law of $T$ uniquely determines that of $S_{T}$, and conversely. For instance, $S_{T}$ has the same distribution as $S_{N}$, for some integer $N$, if and only if $T=N$ a.s. In this case $\psi_{T}(z)=\varphi(z)^{N}$.

(v) Let $T$ be a stopping time satisfying (IP) and $\mathcal{F}_{0}$-measurable, that is, independent of the variables $X_{1}, X_{2}, \ldots$ An elementary computation yields the equality: $\mathbb{E}\left(\exp \lambda S_{T}\right)=\mathbb{E}\left(\varphi(\lambda)^{T}\right)$. Wald's identity becomes

$$
\mathbb{E}\left(\varphi(\lambda)^{T}\right) \mathbb{E}\left(\varphi(\lambda)^{-T}\right)=1,
$$

for every $\lambda \in \mathbb{R}$ such that $\varphi(\lambda) \geq 1$. Now, by Cauchy-Schwarz, $T$ is necessarily constant a.s. The same conclusion holds even without the hypothesis that $T$ is standard. Indeed, by independence of $T$ and $S_{T}$, for any Borel set $A$ and any integer $n$ :

$$
\mathbb{P}\left(S_{T} \in A \text { and } T=n\right)=\mathbb{P}\left(S_{T} \in A\right) \mathbb{P}(T=n) .
$$

On the other hand, $T$ being independent of the $X_{i}$, we have:

$$
\mathbb{P}\left(S_{T} \in A \text { and } T=n\right)=\mathbb{P}\left(S_{n} \in A \text { and } T=n\right)=\mathbb{P}\left(S_{n} \in A\right) \mathbb{P}(T=n) .
$$

Thus,

$$
\forall n \geq 0, \quad \mathbb{P}(T=n)=0 \text { or } S_{T} \sim S_{n},
$$

which means that $T=N$ a.s., for some integer $N$. Note incidentally that this remark applies to the case when $X$ is constant a.s. 
(vi) For a positive, integer-valued random variable $T$, with finite second moment and independent of the $X_{i}$, the following identities are classical:

$$
\mathbb{E} S_{T}=\mu \mathbb{E} T \text { and } \operatorname{Var} S_{T}=\sigma^{2} \mathbb{E} T+\mu^{2} \operatorname{Var} T .
$$

If moreover $T$ satisfies (IP), then (1.4) and (1.5) imply that $\operatorname{Var} T=0$. In other words, we have proved again that $T$ is constant a.s.

Proof of Theorem 1.1. a) For every $\lambda \in]-\alpha, \beta[$, the exponential martingale $\left(M_{n}(\lambda)=\exp \left(\lambda S_{n}\right) / \varphi(\lambda)^{n}, n \geq 0\right)$ is well defined. Since $T$ is standard, we know that $\lim _{n \rightarrow \infty} S_{n \wedge T}=S_{T}$, a.s. and $L^{1}$. Now, by Fatou's lemma,

$$
\mathbb{E}\left(M_{T}(\lambda)\right)=\mathbb{E}\left(\liminf _{n \rightarrow \infty} M_{n \wedge T}(\lambda)\right) \leq \liminf _{n \rightarrow \infty} \mathbb{E}\left(M_{n \wedge T}(\lambda)\right)=1 .
$$

Combining this inequality with the independence assumption, we get

$$
\mathbb{E}\left(M_{T}(\lambda)\right)=\mathbb{E}\left(\exp \lambda S_{T}\right) \mathbb{E}\left(\varphi(\lambda)^{-T}\right) \leq 1 .
$$

From the fact that $\mathbb{E}\left(\varphi(\lambda)^{-T}\right)>0$, we finally deduce (1.2).

Remark 1.3 Another obvious consequence of (1.6) is that $\mathbb{E}\left(\varphi(\lambda)^{-T}\right)<+\infty$, $-\alpha<\lambda<\beta$, else we would have $S_{T}= \pm \infty$ a.s., a contradiction. In the case $\mu \neq 0$, this settles immediately point $\mathrm{d}$ ) of our theorem. In particular, $T$ has then finite moments of any order and remark (1.1) yields the uniform integrability of $\tilde{S}^{T}$.

b) Let us extend the definition of the exponential martingale to complex numbers. More precisely, for every $z \in \mathcal{B}_{\varphi}$ such that $\varphi(z) \neq 0$, we set:

$$
M_{n}(z)=\exp \left(z S_{n}\right) / \varphi(z)^{n}, n \geq 0 .
$$

If $|\varphi(z)| \geq 1$, then $\left|M_{n \wedge T}(z)\right| \leq \exp \left[(\Re e z) S_{n \wedge T}\right]$. We prove first that the dominating process $\left(\exp \left[(\Re e z) S_{n \wedge T}\right], n \geq 0\right)$ is uniformly integrable when $\mu \geq 0$. Two cases have to be distinguished:

$$
\begin{aligned}
& \Re e z \geq 0 \Rightarrow \exp \left[(\Re e z) S_{n \wedge T}\right] \leq \exp \left[(\Re e z) \mathbb{E}\left(S_{T} / \mathcal{F}_{n \wedge T}\right)\right] \\
& \leq \mathbb{E}\left(\exp (\Re e z) S_{T} / \mathcal{F}_{n \wedge T}\right) \\
& \Re e z<0 \Rightarrow \exp \left[(\Re e z) S_{n \wedge T}\right]=\exp \left[(\Re e z) \tilde{S}_{n \wedge T}+(\Re e z)(n \wedge T) \mu\right] \\
& \leq \exp \left[(\Re e z) \tilde{S}_{n \wedge T}\right] \\
&= \exp \left[(\Re e z) \mathbb{E}\left(\tilde{S}_{T} / \mathcal{F}_{n \wedge T}\right)\right] \text { as } \tilde{S}^{T} \text { is u.i. } \\
& \leq \mathbb{E}\left[\exp \left((\Re e z) \tilde{S}_{T}\right) / \mathcal{F}_{n \wedge T}\right] .
\end{aligned}
$$


Inverting the above arguments when $\mu<0$, we conclude that, for any $\mu$, the martingale $M_{n \wedge T}(z)$ is u.i. By the optional stopping theorem we get:

$$
\mathbb{E}\left[\exp \left(z S_{T}\right) / \varphi(z)^{T}\right]=1 .
$$

The independence of $T$ and $S_{T}$ finally leads to (1.3).

c) This is an immediate corollary of b).

d) As already noticed in remark 1.3, the assertion is trivial in the case $\mu \neq 0$. More generally, the function $1 / \psi_{T}(z)$ is analytic at every $z \in \mathcal{B}_{\varphi}$ such that $\psi_{T}(z) \neq 0$. This is true at 0 , since $\psi_{T}(0)=1$. Thus,

$$
\omega_{T}(z)=\mathbb{E}\left(\frac{1}{\varphi(z)^{T}}\right)=\frac{1}{\psi_{T}(z)}
$$

admits analytic continuation in some open disc $D$ centered at 0 . Since $\varphi(D)$ is open and contains 1 , there exists some $\gamma \in] 0,1[\cap \varphi(D)$ for which we have $\mathbb{E}\left[1 / \gamma^{T}\right]<+\infty$.

e) Both identities follow easily from Wald's identity written in terms of the power series expansion of $\psi_{T}$ and $\omega_{T}$ at 0 .

Lemma 1.2 If $T$ satisfies $(I P)$ then either $\mathbb{P}(T=0)=0$ or $\mathbb{P}(T=0)=1$.

Proof. By the independence assumption,

$$
\begin{gathered}
\mathbb{P}\left(\{T=0\} \cap\left\{S_{T}=0\right\}\right)=\mathbb{P}(\{T=0\}) \cdot \mathbb{P}\left(\left\{S_{T}=0\right\}\right) \\
\Leftrightarrow \mathbb{P}(\{T=0\})=\mathbb{P}(\{T=0\}) \cdot \mathbb{P}\left(\left\{S_{T}=0\right\}\right) \\
\Leftrightarrow \mathbb{P}(\{T=0\})=0 \text { or } \mathbb{P}\left(\left\{S_{T}=0\right\}\right)=1 .
\end{gathered}
$$

Now, assume that $S_{T}=0$ a.s. and, for instance, take $\mu \geq 0$. This implies that $0=\mathbb{E}\left(S_{T} / \mathcal{F}_{n \wedge T}\right) \geq S_{n \wedge T}$. On the other hand, since $S^{T}$ is a submartingale, $\mathbb{E}\left(S_{n \wedge T}\right) \geq \mathbb{E}\left(S_{0}\right)=0$. Hence $S_{n \wedge T}=0$ a.s. for every $n$. The same conclusion holds of course in the case $\mu<0$. Observing that the event $\{T \neq 0\}$ belongs to $\mathcal{F}_{0}$, we have:

$$
\begin{gathered}
\mathbb{P}\left(\{T \neq 0\} \cap\left\{X_{1}=0\right\}\right)=\mathbb{P}(\{T \neq 0\}) \cdot \mathbb{P}\left(\left\{X_{1}=0\right\}\right) \\
\Leftrightarrow \mathbb{P}\left(\{T \neq 0\} \cap\left\{S_{1 \wedge T}=0\right\}\right)=\mathbb{P}(\{T \neq 0\}) \cdot \mathbb{P}\left(\left\{X_{1}=0\right\}\right) \\
\Leftrightarrow \mathbb{P}(\{T \neq 0\})=\mathbb{P}(\{T \neq 0\}) \cdot \mathbb{P}\left(\left\{X_{1}=0\right\}\right) .
\end{gathered}
$$

Since the case $X_{1}=0$ a.s. was excluded from the beginning, it follows that $\mathbb{P}(T \neq 0)=0$, i.e. $T=0$ a.s.

Proposition 1.1 Assume that $\varphi$ is an entire function. Let $T \neq 0$ be a $\left(\mathcal{F}_{n}\right)$-stopping time solving $(I P)$. Then:

a) $\varphi$ and $\psi_{T}$ have the same order, which belongs to $[1,+\infty]$.

b) If $\varphi$ is of finite order and of type $\tau$, then the type of $\psi_{T}$ is equal to $\min T \cdot \tau$. 
Proof. Let us first recall the elementary fact that

$$
M_{\varphi}(r):=\sup _{|z| \leq r}|\varphi(z)|=\max \{\varphi(r), \varphi(-r)\}, r \geq 0 .
$$

Furthermore, we note that if $\mathbb{P}(X<0)>0$, then $\lim _{r \rightarrow-\infty} \varphi(r)=+\infty$. Thus, only nonnegative random variables $X$ satisfy $\lim _{r \rightarrow-\infty} \varphi(r)<+\infty$. More precisely, if $X \geq 0$ a.s., then $r \geq 0 \Rightarrow \varphi(r) \geq 1$ and $r \leq 0 \Rightarrow \varphi(r) \leq 1$. Hence

$$
X \geq 0 \text { a.s. } \Rightarrow M_{\varphi}(r)=\varphi(r), \forall r \geq 0
$$

and

$$
X \leq 0 \text { a.s. } \Rightarrow M_{\varphi}(r)=\varphi(-r), \forall r \geq 0 .
$$

Obviously, if $X \geq 0$ a.s. (resp. $X \leq 0$ a.s.) then the same is true for $S_{T}$. Thus, restricted to $\mathbb{R}$, the functions $\varphi$ and $\psi_{T}$ always tend to $+\infty$ together.

Now, set $m=\min T, m \neq 0$ by assumption. For every $\lambda \in \mathbb{R}$ such that $\varphi(\lambda) \geq 1$, Wald's identity yields the following upper bound for $\psi_{T}(\lambda)$ :

$$
\psi_{T}(\lambda)=\frac{1}{\mathbb{E}\left(\varphi(\lambda)^{-T}\right)} \leq \frac{1}{\mathbb{E}\left(\varphi(\lambda)^{-T} \mathbb{I}\{T=m\}\right)} \leq \frac{\varphi(\lambda)^{m}}{\mathbb{P}(T=m)} .
$$

Thus, the order of $\psi_{T}$ is at most equal to the order of $\varphi$. If $\varphi$ is of finite order $\rho$ and of type $\tau$ then the type of $\psi_{T}$ is bounded above by $m \tau$ since

$$
\varlimsup_{|\lambda| \rightarrow+\infty} \frac{\log \psi_{T}(\lambda)}{\lambda^{\rho}} \leq m \varlimsup_{|\lambda| \rightarrow+\infty} \frac{\log \varphi(\lambda)}{\lambda^{\rho}}=m \tau .
$$

On the other hand, when $\varphi(\lambda)>1$ we have

$$
\begin{aligned}
\mathbb{E}\left(\varphi(\lambda)^{-T}\right) & =\sum_{k=m}^{+\infty} \varphi(\lambda)^{-k} P(T=k) \quad(m \neq 0) \\
& \leq \sum_{k=m}^{+\infty} \varphi(\lambda)^{-k}=\frac{\varphi(\lambda)^{-m}}{1-\varphi(\lambda)^{-1}}
\end{aligned}
$$

which yields

$$
\psi_{T}(\lambda)=\frac{1}{\mathbb{E}\left(\varphi(\lambda)^{-T}\right)} \geq \varphi(\lambda)^{m}\left(1-\varphi(\lambda)^{-1}\right) .
$$

This shows that the order of $\psi_{T}$ is at least equal to the order of $\varphi$. If $\varphi$ is of finite order $\rho$ and type $\tau$ then the type of $\psi_{T}$ is bounded below by $m \tau$ since

$$
\varlimsup_{|\lambda| \rightarrow+\infty} \frac{\log \psi_{T}(\lambda)}{\lambda^{\rho}} \geq \varlimsup_{|\lambda| \rightarrow+\infty} \frac{m \log \varphi(\lambda)+\log \left(1-\varphi(\lambda)^{-1}\right)}{\lambda^{\rho}}=m \tau .
$$




\subsection{Conditions which imply the constancy of $T$}

Statement d) of Theorem 1.1 asserts that the radius of convergence $R_{T}$ of the generating function $g_{T}$ is $>1$. Assuming that $R_{T}=\infty$ and imposing some technical constraints on $\varphi$, we are able to show that $T$ is necessarily constant.

Theorem 1.2 Assume that $\varphi$ is an entire function. Let $T$ be a $\left(\mathcal{F}_{n}\right)$-stopping time satisfying (IP). Each of the following conditions:

(a) $T$ is bounded;

$\left(b_{1}\right) \forall r>0, g_{T}(r)<+\infty$ and $\varphi$ has at least one zero;

$\left(b_{2}\right) \forall r>0, g_{T}(r)<+\infty$ and $\varphi$ has finite order

implies the constancy of $T$.

Remark 1.4 (i) In particular, there are no bounded stopping times $T$ such that $T \Perp S_{T}$, except the constant ones.

(ii) If $\varphi$ is of finite order, then it has at least one zero, except for two cases, as will be shown in the lemma below.

(iii) If $\varphi$ is of infinite order, then it does not necessarily vanish, even in the symmetric case. For example, let $X_{1}$ and $X_{2}$ be two independent variables having the Poisson distribution with parameter $\lambda$. Setting $X=X_{1}-X_{2}$, we have $\varphi_{X}(z)=\exp [2 \lambda(\operatorname{ch} z-1)] \neq 0$.

(iv) Open problem: prove Theorem 1.2 without assuming that $\varphi$ has at least one zero.

Lemma 1.3 If $\varphi$ is an entire function of finite order and without any zero, then $X$ is necessarily constant or gaussian.

Proof of Lemma 1.3. By Hadamard's factorization theorem for entire functions of finite order (cf. [13] or [1]), there exists a polynomial $P$ of degree at most the order of $\varphi$, such that $\varphi(z)=\exp (P(z))$. Therefore the statement of the lemma comes immediately from Marcinkiewicz's theorem (cf. [Lu], p. 213).

Proof of Theorem 1.2. Let $\mathcal{Z}_{f}$ denote the set of zeros of an holomorphic function $f$. Our first step is to show that

$$
\mathcal{Z}_{\varphi}=\mathcal{Z}_{\psi_{T}}
$$

whenever the radius of convergence of $g_{T}$ is infinite. Under this assumption, which will be in force throughout the proof, the function $\omega_{T}(z)=\mathbb{E}\left(\varphi(z)^{-T}\right)$ is analytic at each $z$ such that $\varphi(z) \neq 0$. Wald's identity $\psi_{T}(z) \cdot \omega_{T}(z)=1$, 
at the beginning only valid when $|\varphi(z)| \geq 1$, can now be extended on $\mathcal{Z}_{\varphi}^{c}$ by analytic continuation:

$$
\forall z \in \mathcal{Z}_{\varphi}^{c}, \psi_{T}(z) \cdot \omega_{T}(z)=1 .
$$

In particular, if $\varphi(z) \neq 0$ then $\psi_{T}(z) \neq 0$. If $\varphi(z)=0$, the principle of preservation of a domain allows us to choose a sequence of complex numbers $\left(z_{n}\right)$ such that $z_{n} \rightarrow z$ and $\varphi\left(z_{n}\right) \in \mathbb{R}_{+}^{*}$. Then $\omega_{T}\left(z_{n}\right)$ tends to infinity and by (1.9), $\psi_{T}\left(z_{n}\right)$ tends to 0 , whence $\psi_{T}(z)=0$. Thus (1.8) holds.

Now, assume that $\mathcal{Z}_{\varphi} \neq \emptyset$. This implies that $T$ is necessarily bounded. Indeed, rewrite (1.9) as follows:

$$
\forall z \in \mathcal{Z}_{\varphi}^{c}, \omega_{T}(z)=\frac{1}{\psi_{T}(z)},
$$

and observe that the righthand side of the last equality is a meromorphic function. Given $z_{0} \in \mathcal{Z}_{\varphi}$, (1.8) ensures the existence of an integer $N$ such that the function

$$
\mathbb{E}\left(\varphi(z)^{N-T}\right)=\frac{\varphi(z)^{N}}{\psi_{T}(z)}
$$

is holomorphic in some neighbourhood of $z_{0}$. On the other hand,

$$
\mathbb{E}\left(\varphi(z)^{N-T}\right)=\sum_{k=0}^{+\infty} \varphi(z)^{N-k} p_{k}
$$

where $p_{k}=\mathbb{P}(T=k)$. Since $\lim _{z \rightarrow z_{0}} \varphi(z)=0$, we may choose again a sequence $\left(z_{n}\right)$ such that $z_{n} \rightarrow z_{0}, \varphi\left(z_{n}\right) \in \mathbb{R}_{+}^{*}$ and by continuity, $\varphi\left(z_{n}\right) \rightarrow 0$. If $p_{l} \neq 0$ for some $l>N$, we would have

$$
\lim _{n \rightarrow \infty} \mathbb{E}\left(\varphi\left(z_{n}\right)^{N-T}\right) \geq \lim _{n \rightarrow \infty} \varphi\left(z_{n}\right)^{N-l} p_{l}=+\infty,
$$

a contradiction. Hence $T \leq N$ a.s.

We finally prove that $T$ is in fact constant. Set $N=\max T$ and $n=$ $\min T$, so that in particular $p_{N} \neq 0$ and $p_{n} \neq 0$. For every $z \in \mathcal{Z}_{\varphi}^{c}$, we have

$$
\begin{aligned}
\omega_{T}(z) & =\mathbb{E}\left(\varphi(z)^{-T}\right) \\
& =\sum_{k=n}^{N} \frac{p_{k}}{\varphi(z)^{k}}=\frac{1}{\varphi(z)^{N}} \sum_{k=n}^{N} p_{k} \varphi(z)^{N-k}=\frac{P(\varphi(z))}{\varphi(z)^{N}},
\end{aligned}
$$

where $P$ is a polynomial of degree $N-n$ such that $P(0) \neq 0$. Combining (1.10) and (1.11) yields:

$$
\forall z \in \mathcal{Z}_{\varphi}^{c}, \frac{P(\varphi(z))}{\varphi(z)^{N}}=\frac{1}{\psi_{T}(z)} .
$$


This shows that $P(\varphi(z))$ never vanishes. Thus, inverting both sides, we get:

$$
\forall z \in \mathbb{C}, \psi_{T}(z)=\frac{\varphi(z)^{N}}{P(\varphi(z))},
$$

whence

$$
\forall z \in \mathcal{Z}_{\varphi}^{c}, \frac{\psi_{T}(z)}{\varphi(z)^{n}}=\frac{\varphi(z)^{N-n}}{P(\varphi(z))} .
$$

It is easy to see that the righthand side of the last equality is a bounded entire function, so necessarily constant by Liouville's theorem. Since $\psi_{T}(0)=$ $\varphi(0)=1$, we have $\psi_{T}(z)=\varphi(z)^{n}$. This means that $T=n$ a.s., by Remark 1.2, (iv).

It remains to study the Gaussian case, i.e. $\varphi(z)=\exp \left(\mu z+\frac{\sigma^{2}}{2} z^{2}\right)$, with $\sigma \neq 0$. The other cases are settled thanks to Remark 1.4, (ii) and the first step of the proof. The generating function of $T$ being entire, we derive from (1.8) and statement a) of Proposition 1.1 that $\psi_{T}$ is an entire function of order 2 without zero. Thus, by Lemma 1.3, $\psi_{T}$ is either gaussian or constant, that is: $\psi_{T}(z)=\exp \left(a z+b z^{2}\right)$, where $a=\mathbb{E} S_{T}$ and $b=\operatorname{Var} S_{T} / 2$. Combining Wald's identity and the relations in (1.4), we have

$$
\mathbb{E}\left[\exp \left(-\left(\mu z+\frac{\sigma^{2}}{2} z^{2}\right) T\right)\right]=\exp \left(-\left(\mu z+\frac{\sigma^{2}}{2} z^{2}\right) \mathbb{E} T+\frac{\mu^{2}}{2} z^{2} \operatorname{Var} T\right),
$$

or equivalently:

(1.12) $\mathbb{E}\left[\exp \left(-\left(\mu z+\frac{\sigma^{2}}{2} z^{2}\right) \hat{T}\right)\right]=\exp \left(\frac{\mu^{2}}{2} z^{2} \operatorname{Var} T\right)$, where $\hat{T}=T-\mathbb{E} T$.

If $\mu \neq 0$, the equation $\mu z+\frac{\sigma^{2}}{2} z^{2}=0$ admits $z_{0}=-2 \mu / \sigma^{2}>0$ as a solution. Substituting $z=z_{0}$ in $(1.12)$, we get $1=\exp \left(2 \mu^{4} \operatorname{Var} T / \sigma^{4}\right)$, which is only possible if $\operatorname{Var} T=0$, i.e. if $T$ is constant. If $\mu=0$ then (1.12) becomes:

$$
\mathbb{E}\left[\exp \left(-\frac{\sigma^{2}}{2} z^{2} \hat{T}\right)\right]=1, \forall z \in \mathbb{C} .
$$

from which follows at once that $\hat{T}=0$ a.s., i.e. $T=\mathbb{E} T$ a.s.

\subsection{Moment inequalities}

We begin with an upper bound for $\mathbb{E}\left(S_{T}^{4}\right)$.

Proposition 1.2 Assume that $X$ is symmetric, $\sigma^{2}=\mathbb{E}\left(X^{2}\right)$ and $\tau^{4}=\mathbb{E}\left(X^{4}\right)$. Let $T$ be a $\left(\mathcal{F}_{n}\right)$-stopping time satisfying $(I P)$. Then

$$
\mathbb{E}\left(S_{T}^{2}\right)=\sigma^{2} \mathbb{E} T
$$

and

$$
\mathbb{E}\left(S_{T}^{4}\right) \leq 3 \sigma^{4}(\mathbb{E} T)^{2}-\left(3 \sigma^{4}-\tau^{4}\right) \mathbb{E} T,
$$

with equality if and only if $T$ is constant. 
Proof. Equality (1.13) is just the second identity in (1.4). In order to prove (1.14), one shows easily that

$$
\left(M_{n}=S_{n}^{4}-6 n \sigma^{2} S_{n}^{2}+3 n(n+1) \sigma^{4}-n \tau^{4} ; n \geq 0\right)
$$

is a martingale and then applies the optional stopping theorem:

$\mathbb{E}\left(S_{n \wedge T}^{4}\right)=6 \sigma^{2} \mathbb{E}\left[(n \wedge T) S_{n \wedge T}^{2}\right]-3 \sigma^{4} \mathbb{E}[(n \wedge T)(n \wedge T+1)]+\tau^{4} \mathbb{E}(n \wedge T)$.

Since $T$ has finite moments of any order, we may let $n$ tend to $+\infty$. The independence assumption then yields:

$$
\mathbb{E}\left(S_{T}^{4}\right)=6 \sigma^{2} \mathbb{E} T \mathbb{E}\left(S_{T}^{2}\right)-3 \sigma^{4} \mathbb{E}\left(T^{2}+T\right)+\tau^{4} \mathbb{E} T .
$$

Combining this with (1.13), we get:

$$
\mathbb{E}\left(S_{T}^{4}\right)=3 \sigma^{4}(\mathbb{E} T)^{2}-3 \sigma^{4} \operatorname{Var} T-\left(3 \sigma^{4}-\tau^{4}\right) \mathbb{E} T,
$$

from which (1.14) follows readily.

Remark 1.5 When $\mathbb{P}(X=1)=\mathbb{P}(X=-1)=1 / 2$, (1.14) simplifies to

$$
\mathbb{E}\left(S_{T}^{4}\right) \leq 3(\mathbb{E} T)^{2}-2 \mathbb{E} T=3\left(\mathbb{E} S_{T}^{2}\right)^{2}-2 \mathbb{E} S_{T}^{2} .
$$

In particular, Khinchine's well-known inequality $\mathbb{E}\left(S_{T}^{4}\right) \leq 3\left(\mathbb{E} S_{T}^{2}\right)^{2}$ holds. In what follows, we will generalize this inequality for moments of higher order.

Let us recall that a random variable $X$ satisfies "Khinchine's inequalities" if

$$
\mathbb{E}\left(X^{2 p}\right) \leq \frac{(2 p) !}{2^{p} p !} \mathbb{E}\left(X^{2}\right)^{p}, \forall p \in \mathbb{N} .
$$

These inequalities hold for example if $X$ is distributed according to the symmetric binomial distribution with parameter $n$, denoted by $\mathcal{B S}(n)$,

$$
X \sim \mathcal{B S}(n) \Longleftrightarrow \varphi_{X}(z)=(\operatorname{ch} z)^{n},
$$

or, more generally, if $X$ belongs to Newman's class $(\mathcal{L})$ (cf. [9]), i.e. the class of random variables whose Laplace transform $\varphi$ has the following properties:

i) $\varphi$ is of order at most 2 ,

ii) the zeros of $\varphi$ are all purely imaginary.

$X$ is said to satisfy "Khinchine's improved inequalities" (for large moments), if there exist constants $C>0$ and $\alpha \in] 0,1[$ such that:

$$
\mathbb{E}\left(X^{2 p}\right) \leq C \alpha^{p} \frac{(2 p) !}{2^{p} p !}\left[\mathbb{E}\left(X^{2}\right)\right]^{p}, \forall p \in \mathbb{N} .
$$


Proposition 1.3 Assume that $X$ is centered and belongs to Newman's class $(\mathcal{L})$. Let $T$ be a $\left(\mathcal{F}_{n}\right)$-stopping time satisfying $(I P)$. Then:

$$
\mathbb{E}\left(S_{T}^{2 p}\right) \leq C \alpha^{p} \frac{(2 p) !}{2^{p} p !}\left(\mathbb{E}\left(S_{T}^{2}\right)\right)^{p}, \forall p \in \mathbb{N}
$$

where $C=1 / \mathbb{P}(T=m)$ and $\alpha=m / \mathbb{E}(T), m$ being the minimum of $T$.

In particular, $S_{T}$ satisfies Khinchine's improved inequalities if and only if $T$ is not constant. lemma.

The key tool for proving (1.17) is contained in the following elementary

Lemma 1.4 If $X$ is centered and $T$ satisfies (IP), then

$$
\forall n, p \in \mathbb{N}, \quad \mathbb{P}(T \leq n) \mathbb{E}\left(S_{T}^{2 p}\right) \leq \mathbb{E}\left(S_{n}^{2 p}\right) .
$$

Proof of Lemma 1.4. Under the above assumptions, $\left(S_{n}^{2 p}, n \geq 0\right)$ is a submartingale. By the optional stopping theorem and the independence hypothesis, we have successively:

$$
\begin{aligned}
\mathbb{E}\left(S_{n}^{2 p}\right) & \geq \mathbb{E}\left(S_{n \wedge T}^{2 p}\right) \geq \mathbb{E}\left(S_{n \wedge T}^{2 p} \mathbb{I}\{T \leq n\}\right) \\
& =\mathbb{E}\left(S_{T}^{2 p} \mathbb{I}\{T \leq n\}\right)=\mathbb{E}\left(S_{T}^{2 p}\right) \mathbb{P}(T \leq n) .
\end{aligned}
$$

Proof of Proposition 1.3. We apply Lemma 1.4 with $n=m=\min T$ :

$$
\mathbb{E}\left(S_{T}^{2 p}\right) \leq \frac{1}{\mathbb{P}(T \leq m)} \cdot \mathbb{E}\left(S_{m}^{2 p}\right)
$$

Now, since $X$ belongs to Newman's class $(\mathcal{L})$, the same is clearly true for any finite sum of independent random variables distributed as $X$. Thus, each $S_{n}, n \geq 0$, belongs to $(\mathcal{L})$. By Khinchine's inequality,

$$
\begin{aligned}
\mathbb{E}\left(S_{T}^{2 p}\right) & \leq \frac{1}{\mathbb{P}(T \leq m)} \frac{(2 p) !}{2^{p} p !}\left[\mathbb{E}\left(S_{m}^{2}\right)\right]^{p} \\
& =\frac{1}{\mathbb{P}(T \leq m)} \frac{(2 p) !}{2^{p} p !}\left(m \sigma^{2}\right)^{p} \\
& =\frac{1}{\mathbb{P}(T \leq m)}\left(\frac{m}{\mathbb{E} T}\right)^{p} \frac{(2 p) !}{2^{p} p !}\left(\sigma^{2} \mathbb{E} T\right)^{p} .
\end{aligned}
$$

Since $\mathbb{E}\left(S_{T}^{2}\right)=\sigma^{2} \mathbb{E} T$, inequality (1.17) is proved. It remains to observe that the constant $\alpha=m / \mathbb{E} T$ is $<1$ if and only if $T$ is not constant, whence the last assertion of the proposition follows immediately. 
Proposition 1.4 Assume that $X$ is centered. Let $T$ be a stopping time satisfying (IP) and set $m=\min T$. Then, denoting by $\|.\|_{p}$ the norm in $\mathcal{L}^{p}(\Omega, \mathcal{F}, \mathbb{P})$, we have:

$$
\begin{aligned}
\left\|S_{T}\right\|_{2 p} \leq & \frac{m}{\mathbb{P}(T=m)^{1 / 2 p}}\|X\|_{2 p}, p \in \mathbb{N}^{*} \\
& \left\|S_{T}\right\|_{\infty} \leq m\|X\|_{\infty} .
\end{aligned}
$$

Proof. Once more, we apply Lemma 1.4 with $n=m=\min T$ :

$$
\left\|S_{T}\right\|_{2 p} \leq \frac{1}{\mathbb{P}(T=m)^{1 / 2 p}}\left\|S_{m}\right\|_{2 p} \leq \frac{m}{\mathbb{P}(T=m)^{1 / 2 p}}\|X\|_{2 p},
$$

since

$$
\left\|S_{m}\right\|_{2 p}=\left\|X_{1}+\ldots+X_{m}\right\|_{2 p} \leq m\|X\|_{2 p} .
$$

Letting $p$ tend to $+\infty$, we obtain (1.19).

We end this section by refining (1.19).

Proposition 1.5 Assume that $X$ is bounded. Let $T$ be a stopping time (standard or not) independent of $S_{T}$. Then (1.19) holds with $m=\min T$. If moreover $T$ is standard and $X$ is centered, then (1.19) becomes an equality.

Proof. Let $m=\min T$ and $M=\|X\|_{\infty}$. By the independence assumption:

$$
\mathbb{P}\left(T=m \text { and }\left|S_{T}\right|>m M\right)=\mathbb{P}(T=m) \mathbb{P}\left(\left|S_{T}\right|>m M\right) .
$$

But

$$
\mathbb{P}\left(T=m \text { and }\left|S_{T}\right|>m M\right) \leq \mathbb{P}\left(\left|S_{m}\right|>m M\right)=0
$$

and $\mathbb{P}(T=m) \neq 0$. Thus, $\mathbb{P}\left(\left|S_{T}\right|>m M\right)=0$, which means that

$$
\left\|S_{T}\right\|_{\infty} \leq \min T \cdot\|X\|_{\infty}
$$

Assuming moreover that $T$ is standard and $X$ is centered, we know that $\left|S_{n \wedge T}\right| \leq m M$, for every $n$. To prove the second statement of the proposition, choose $0<\varepsilon<\frac{m M}{m+1}$ and write:

$$
\begin{aligned}
0 & <\mathbb{P}\left(X_{1}>M-\frac{\varepsilon}{m}, X_{2}>M-\frac{\varepsilon}{m}, \ldots, X_{m+1}>M-\frac{\varepsilon}{m}\right) \\
& \leq \mathbb{P}\left(T=m \text { and } S_{T}>m M-\varepsilon\right)\left(\text { else } S_{m+1}=S_{T \wedge(m+1)}>m M, \text { impossible }\right) \\
& =\mathbb{P}(T=m) \cdot \mathbb{P}\left(S_{T}>m M-\varepsilon\right) .
\end{aligned}
$$

Hence, for $\varepsilon$ small enough, we have $\mathbb{P}\left(S_{T}>m M-\varepsilon\right)>0$. 


\section{Examples}

\subsection{Some general results in the case of a Bernoulli random walk}

Throughout this section, we denote by $T$ a $\left(\mathcal{F}_{n}\right)$-stopping time solving (IP) and by $m$ its minimum. Most of the time, we will work under the assumption that $S$ is a Bernoulli random walk, that is:

$$
\mathbb{P}(X=1)=p \text { and } \mathbb{P}(X=-1)=q=1-p,
$$

for some $p \in] 0,1[$. In the symmetric case, i.e. $p=1 / 2$, Proposition 1.5 shows that

$$
\left\|S_{T}\right\|_{\infty}=\min T=m .
$$

The following lemma gives further details in the general case, i.e. $0<p<1$.

Lemma 2.1 In the general Bernoulli case and with the above notation, we have:

$$
\begin{aligned}
& \operatorname{Supp}\left(\mathcal{L}\left(S_{T}\right)\right) \subset\{m-2 j, 0 \leq j \leq m\}, \\
& \operatorname{Supp}(\mathcal{L}(T)) \subset\{m+2 k, k \geq 0\}, \\
& \mathbb{P}\left(S_{T}=m\right)>0 \text { and } \mathbb{P}\left(S_{T}=-m\right)>0 .
\end{aligned}
$$

Proof. Since $T \Perp S_{T}$, we have

$$
\mathbb{P}\left(S_{T}=k\right)=\mathbb{P}\left(S_{T}=k / T=m\right)=\mathbb{P}\left(S_{m}=k \text { and } T=m\right) / \mathbb{P}(T=m)
$$

and similarly,

$\mathbb{P}(T=k)=\mathbb{P}\left(T=k /\left|S_{T}\right|=m\right)=\mathbb{P}\left(T=k\right.$ and $\left.\left|S_{k}\right|=m\right) / \mathbb{P}\left(\left|S_{T}\right|=m\right)$.

Whenever these probabilities are $\neq 0, m$ and $k$ must have the same parity, whence (2.2) and (2.3) follow. To show (2.4), we proceed as in Proposition 1.5:

$$
\begin{aligned}
0 & <\mathbb{P}\left(X_{1}=\cdots=X_{m}=X_{m+1}=1\right) \\
& \leq \mathbb{P}\left(T=m \text { and } S_{T}=m\right) \\
& \leq \mathbb{P}\left(S_{T}=m\right) .
\end{aligned}
$$

The positivity of $\mathbb{P}\left(S_{T}=-m\right)$ can be obtained in the same way.

Notational convention Every time we work with the Bernoulli random walk, characters topped by $a \sim$ (resp. without the $\sim)$ will refer to quantities computed in the symmetric (resp. general) case. 
Lemma 2.2 In the symmetric Bernoulli case, there exists a polynomial $\tilde{P}_{m}$ of degree $m=\min T$, such that:

$$
\begin{aligned}
& \tilde{\psi}_{T}(z)=\tilde{\mathbb{E}}\left(\exp z S_{T}\right)=\tilde{P}_{m}(\operatorname{ch} z) \\
& \tilde{g}_{T}(r)=\tilde{\mathbb{E}}\left(r^{T}\right)=\frac{1}{\tilde{P}_{m}(1 / r)}
\end{aligned}
$$

Moreover, $\tilde{P}_{m}(1)=1, \tilde{P}_{m}$ has the same parity as $m$ and all the roots of $\tilde{P}_{m}$ are located in the open unit disk.

Proof. Relation (2.5), as well as the degree and the parity of $\tilde{P}_{m}$, are easily derived from (2.2) and the symmetry of $S_{T}$. (2.6) is an immediate consequence of Wald's identity. Point c) of Theorem 1.1 states that if $|\operatorname{ch} z| \geq 1$, then $\left|\tilde{\psi}_{T}(z)\right| \geq 1$, so that $\tilde{P}_{m}(\operatorname{ch} z) \neq 0$. Since the equation $\operatorname{ch} z=u$, where $|u| \geq 1$, has always at least one solution $z$, the roots of $\tilde{P}_{m}$ are necessarily located in the open unit disk.

So far, the filtration $\left(\mathcal{F}_{n}, n \geq 0\right)$ has not played a significant role in our discussion. Clearly, if the filtration is assumed to be natural, that is,

$$
\mathcal{F}_{0}=\{\Omega, \emptyset\} \text { and } \mathcal{F}_{n}=\sigma\left(X_{1}, X_{2}, \ldots, X_{n}\right), n \geq 1,
$$

then the class of stopping times solving (IP) is more restricted. This will become apparent for instance by comparing example 4 (cf. proposition 2.6) with its "natural" analogue (cf. proposition 3.3). In the framework of Bernoulli random walks, hypothesis (2.7) leads to a more surprising result, namely: the independence of $T$ and $S_{T}$ under $\tilde{\mathbb{P}}$, the probability associated with the symmetric case $(p=1 / 2)$, suffices to ensure independence in the general case $(0<p<1)$.

Proposition 2.1 Let $T$ be a stopping time in the natural filtration of a Bernoulli random walk, with minimum $m$. If $T$ satisfies (IP) in the symmetric case $(p=1 / 2)$, the same is true in the general case $(0<p<1)$. Moreover, setting $\varphi(z)=p e^{z}+q e^{-z}$ and denoting by $\tilde{P}_{m}$ the polynomial of Lemma 2.2, we have:

$$
\begin{aligned}
& \psi_{T}(z)=\tilde{P}_{m}\left(\frac{\varphi(z)}{2 \sqrt{p q}}\right) / \tilde{P}_{m}\left(\frac{1}{2 \sqrt{p q}}\right), \\
& g_{T}(r)=\tilde{P}_{m}\left(\frac{1}{2 \sqrt{p q}}\right) / \tilde{P}_{m}\left(\frac{1}{2 \sqrt{p q} \cdot r}\right) .
\end{aligned}
$$


Proof. Write $\Omega_{n}$ for the set of all $2^{n}$ possible trajectories of the walk until time $n$. Consider the event $\Gamma_{j, k}:=\left\{S_{T}=m-2 j, T=m+2 k\right\} \in \mathcal{F}_{m+2 k}$. By (2.7), it is possible to identify it in a natural way with a subset of $\Omega_{m+2 k}$. Observing that the number of positive and negative jumps of a trajectory in $\Gamma_{j, k}$ is respectively $m+k-j$ and $k+j$, we have:

$$
\mathbb{P}\left(S_{T}=m-2 j, T=m+2 k\right)=\left|\Gamma_{j, k}\right| p^{m+k-j} q^{k+j} .
$$

In particular, when $p=q=1 / 2$, this becomes:

$$
\tilde{\mathbb{P}}\left(S_{T}=m-2 j, T=m+2 k\right)=\left|\Gamma_{j, k}\right|\left(\frac{1}{2}\right)^{m+2 k} .
$$

Combining the previous equalities and using the independence of $T$ and $S_{T}$ under $\tilde{\mathbb{P}}$, we get:

$$
\begin{aligned}
\mathbb{P}\left(S_{T}\right. & =m-2 j, T=m+2 k) \\
& =\tilde{\mathbb{P}}\left(S_{T}=m-2 j\right) \tilde{\mathbb{P}}(T=m+2 k)(2 p)^{m+k-j}(2 q)^{k+j} .
\end{aligned}
$$

From this, we derive the laws of $S_{T}$ and $T$ under $\mathbb{P}$ in terms of the corresponding laws under $\tilde{\mathbb{P}}$ :

$$
\begin{aligned}
& \mathbb{P}\left(S_{T}=m-2 j\right)=\left(\frac{p}{q}\right)^{\frac{m-2 j}{2}} \tilde{\mathbb{P}}\left(S_{T}=m-2 j\right) \sum_{k}(2 \sqrt{p q})^{m+2 k} \tilde{\mathbb{P}}(T=m+2 k) \\
& (2.11) \quad=\left(\frac{p}{q}\right)^{\frac{m-2 j}{2}} \tilde{g}_{T}(2 \sqrt{p q}) \tilde{\mathbb{P}}\left(S_{T}=m-2 j\right)
\end{aligned}
$$

and:

$$
\begin{aligned}
\mathbb{P}(T=m+2 k) & =(4 p q)^{\frac{m}{2}+k} \tilde{\mathbb{P}}(T=m+2 k) \sum_{j=0}^{m} \tilde{\mathbb{P}}\left(S_{T}=m-2 j\right)\left(\frac{p}{q}\right)^{\frac{m}{2}-j} \\
& =(4 p q)^{\frac{m}{2}+k} \tilde{\mathbb{P}}(T=m+2 k) \tilde{\psi}_{T}\left(\frac{1}{2} \log \left(\frac{p}{q}\right)\right) \\
(2.12) \quad & =\frac{(4 p q)^{\frac{m}{2}+k} \tilde{\mathbb{P}}(T=m+2 k)}{\tilde{g}_{T}(2 \sqrt{p q})},
\end{aligned}
$$

where the last equality is just Wald's identity in the symmetric case. The independence of $S_{T}$ and $T$ under $\mathbb{P}$ now easily follows from (2.10), (2.11) and (2.12). Another application of Wald's identity and some straightforward computations lead to (2.8) and (2.9). Let us finally notice that $T$ is also standard under $\mathbb{P}$. Indeed, by (2.9), the radius of convergence of the generating function $g_{T}$ is $>1$ and therefore $T$ has a finite expectation under $\mathbb{P}$. 
To end this subsection, we state the following combinatorial lemma without proof. A more general result may be found in [4], chapter III.10, problem 3, page 96 .

Lemma 2.3 Given two nonnegative integers $a$ and $b$, denote by $\alpha_{j}^{a, b}$ the number of paths in $\mathbb{Z}$, which lead from 0 to a in $a+2 j$ steps, without leaving $\{-b, \ldots, a\}$. Then:

$$
\alpha_{j}^{a, b}=\sum_{k \in \mathbb{Z}} C_{a+2 j}^{j+k(a+b+2)}-C_{a+2 j}^{j-1+k(a+b+2)} .
$$

\subsection{Example 1: The stopping time $T_{a}^{*}$}

Let $T_{a}^{*}=\inf \left\{n:\left|S_{n}-S_{0}\right| \geq a\right\}$. The following proposition shows that, if $T_{a}^{*} \Perp S_{T_{a}^{*}}, \forall a \geq 0$, then the support of the distribution of $X$ is very particular.

Proposition 2.2 Assume that $X$ is bounded. Then the following are equivalent:

(i) for every $a \geq 0, T_{a}^{*}$ and $S_{T_{a}^{*}}$ are independent;

(ii) $\operatorname{supp} \mathcal{L}(X) \subset\{-\lambda, 0, \lambda\}$.

Remark 2.1 The assumption that $X$ is bounded is essential in our proof. We do not know whether or not, there exists an unbounded variable $X$, for which the independence of $T_{a}^{*}$ and $S_{T_{a}^{*}}$ holds for every $a$.

Proof of Proposition 2.2. (i) $\Rightarrow$ (ii): Denote by $\lambda$ and $\kappa$ respectively the maximum and the minimum of the support of the law of $X$. Assume for instance that $\lambda>0$. Then, for every $a \in] 0, \lambda[$, we have

$$
\mathbb{P}\left(T_{a}^{*}=1 \text { and } S_{T_{a}^{*}}>\lambda\right)=\mathbb{P}\left(X_{1}>\lambda\right)=0 .
$$

Since $\mathbb{P}\left(T_{a}^{*}=1\right) \geq \mathbb{P}\left(X_{1} \geq a\right) \neq 0$, it follows from the independence assumption that $\mathbb{P}\left(S_{T_{a}^{*}}>\lambda\right)=0$. But,

$$
\forall \varepsilon \in] 0, a\left[, \quad \mathbb{P}\left(S_{T_{a}^{*}}>\lambda\right) \geq \mathbb{P}\left(\varepsilon<X_{1}<a\right) \mathbb{P}\left(X_{2}>\lambda-\varepsilon\right)\right.
$$

and, by definition, $\mathbb{P}\left(X_{2}>\lambda-\varepsilon\right) \neq 0$. Hence $\mathbb{P}\left(\varepsilon<X_{1}<a\right)=0$. Letting $a$ tend to $\lambda$ and $\varepsilon$ to 0 , we obtain that

$$
\mathbb{P}(X \in] 0, \lambda[)=0 .
$$

A similar argument shows that, if $\kappa<0$, then

$$
\mathbb{P}(X \in] \kappa, 0[)=0 .
$$


To sum up, we have proved that:

$$
\operatorname{Supp} \mathcal{L}(X) \subset\{\kappa, 0, \lambda\},
$$

where the real numbers $\kappa, 0$ and $\lambda$ are not necessarily distinct. If $\kappa=0$ or $\kappa=\lambda$, there is nothing more to prove. So, let us assume that $\kappa<0<\lambda$. The goal is then to show that $\kappa=-\lambda$. If for instance, $0<|\kappa|<\lambda$, then we would have:

$$
\mathbb{P}\left(T_{\lambda}^{*}=1 \text { and } S_{T_{\lambda}^{*}}<0\right)=\mathbb{P}\left(T_{\lambda}^{*}=1\right) \cdot \mathbb{P}\left(S_{T_{\lambda}^{*}}<0\right)=0 .
$$

But,

$$
\mathbb{P}\left(T_{\lambda}^{*}=1\right)=\mathbb{P}\left(X_{1}=\lambda\right) \neq 0
$$

and,

$$
\mathbb{P}\left(S_{T_{\lambda}^{*}}<0\right) \geq \mathbb{P}\left(X_{1}=\kappa\right) \cdot \mathbb{P}\left(X_{2}=\kappa\right) \cdot \ldots \cdot \mathbb{P}\left(X_{\nu}=\kappa\right) \neq 0,
$$

where $\nu$ is an integer such that $\nu \cdot|\kappa| \geq \lambda$. The required contradiction arises when comparing the two last statements with (2.13). The case where $|\kappa|>\lambda$ may be handled in an analogous way.

(ii) $\Rightarrow$ (i): We may restrict to the case where $\lambda=1$ and $a \in \mathbb{N}^{*}$. If $\mathbb{P}(X=0)=0$, then $S$ is a Bernoulli random walk. The symmetric case $(p=1 / 2)$ is trivial, but suffices, by Proposition 2.1, to settle the general case. If $\mathbb{P}(X=0)>0$, a direct proof leads easily to the independence.

In order to get explicit expressions of $g_{T_{a}^{*}}$ and $\psi_{T_{a}^{*}}$ in the general Bernoulli case, we adopt a direct approach:

$$
\left\{\begin{array}{l}
\mathbb{P}\left(T_{a}^{*}=a+2 j \text { and } S_{T_{a}^{*}}=a\right)=\beta_{j}^{a} p^{a+j} q^{j} \\
\mathbb{P}\left(T_{a}^{*}=a+2 j \text { and } S_{T_{a}^{*}}=-a\right)=\beta_{j}^{a} p^{j} q^{a+j},
\end{array}\right.
$$

where $\beta_{j}^{a}$ denotes the number of paths of length $a+2 j$ in $\mathbb{Z}$ such that $T_{a}^{*}=a+2 j$ and $S_{T_{a}^{*}}=a$. By Lemma 2.3, we have

$$
\beta_{j}^{a}=\alpha_{j}^{a-1, a-1}=\sum_{k \in \mathbb{Z}} C_{a-1+2 j}^{j+2 k a}-C_{a-1+2 j}^{j-1+2 k a} .
$$

Thus, the law of $T_{a}^{*}$ is given by

$$
\mathbb{P}\left(T_{a}^{*}=a+2 j\right)=\beta_{j}^{a} p^{j} q^{j}\left(p^{a}+q^{a}\right) .
$$

In particular, notice that

$$
\sum_{j=0}^{+\infty} \beta_{j}^{a}(p q)^{j}=\frac{1}{\left(p^{a}+q^{a}\right)} .
$$

The distribution of $S_{T_{a}^{*}}$ is classical (cf. ruin problem, [4], ch. XIV.2, p. 344):

$$
\mathbb{P}\left(S_{T_{a}^{*}}=a\right)=\frac{p^{a}}{p^{a}+q^{a}} \text { and } \mathbb{P}\left(S_{T_{a}^{*}}=-a\right)=\frac{q^{a}}{p^{a}+q^{a}} .
$$


Corollary 2.1 Take $\varphi(z)=p e^{z}+q e^{-z}$ and denote by $\tilde{C}_{a}, a \geq 0$, the Chebyshev polynomials of the first kind. Then

$$
\begin{aligned}
& \psi_{T_{a}^{*}}(z)=\frac{2 \sqrt{p^{a} q^{a}}}{p^{a}+q^{a}} \cdot \tilde{C}_{a}\left(\frac{\varphi(z)}{2 \sqrt{p q}}\right), \\
& g_{T_{a}^{*}}(r)=\frac{p^{a}+q^{a}}{2 \sqrt{p^{a} q^{a}}} \cdot \frac{1}{\tilde{C}_{a}\left(\frac{1}{2 \sqrt{p q} r}\right)} .
\end{aligned}
$$

In the symmetric case $(p=q=1 / 2)$, (2.15) and (2.16) simplify to

$$
\tilde{\psi}_{T_{a}^{*}}(z)=\tilde{C}_{a}(\operatorname{ch} z) \quad \text { and } \quad \tilde{g}_{T_{a}^{*}}(r)=\frac{1}{\tilde{C}_{a}\left(\frac{1}{r}\right)}=\sum_{j=0}^{+\infty} 2 \beta_{j}^{a}\left(\frac{r}{2}\right)^{a+2 j} .
$$

Proof. The symmetric case is straightforward. (2.15) and (2.16) follow easily from Proposition 2.1 and (2.14).

Remark 2.2 (i) The roots of $\tilde{C}_{a}$ are given by $\cos \left(\frac{2 k-1}{2 a} \pi\right), k=1, \ldots, a$. Thus, the radius of convergence of $g_{T_{a}^{*}}$ is equal to $1 / 2 \sqrt{p q} \cos \frac{\pi}{2 a}>1$.

(ii) In the symmetric case, another expression of the law of $T_{a}^{*}$ can be derived from the power series expansion of $g_{T_{a}^{*}}(r)=1 / \tilde{C}_{a}(1 / r)$, provided that $a$ is fairly small. The following table gives our results for $a \leq 5$.

\begin{tabular}{|c|c|c|}
\hline$a$ & $g_{T_{a}^{*}}(r)$ & $\mathcal{L}\left(T_{a}^{*}\right)$ \\
\hline 2 & $\frac{r^{2}}{2-r^{2}}$ & $\mathbb{P}\left(T_{2}^{*}=2+2 k\right)=\frac{1}{2^{k+1}}, k \geq 0$ \\
\hline 3 & $\frac{r^{3}}{4-3 r^{2}}$ & $\mathbb{P}\left(T_{3}^{*}=3+2 k\right)=\frac{1}{4}\left(\frac{3}{4}\right)^{k}, k \geq 0$ \\
\hline 4 & $\frac{r^{4}}{r^{4}-8 r^{2}+8}$ & $\mathbb{P}\left(T_{4}^{*}=4+2 k\right)=\frac{1}{8} \sum_{\nu} C_{k-\nu}^{\nu}\left(-\frac{1}{8}\right)^{\nu}, k \geq 0$ \\
\hline 5 & $\frac{r^{5}}{5 r^{4}-20 r^{2}+16}$ & $\mathbb{P}\left(T_{5}^{*}=5+2 k\right)=\frac{1}{16}\left(\frac{5}{4}\right)^{k} \sum_{\nu} C_{k-\nu}^{\nu}\left(-\frac{1}{5}\right)^{\nu}, k \geq 0$. \\
\hline
\end{tabular}

\subsection{Example 2: J. Pitman's example}

Here we work again with a Bernoulli random walk in its natural filtration, that is, (2.1) and (2.7) are in force. Following Pitman (cf. [10]), we define $M_{n}=\sup _{0 \leq k \leq n} S_{k}$ and $Y_{n}=2 M_{n}-S_{n}$. Note that $Y_{n}$ is the symmetric of $S_{n}$ with respect to $M_{n}$.

It is easy to see that the 2 -dimensional process $\left(\left(M_{n}, Y_{n}\right), n \geq 0\right)$ is Markovian on the state space $\mathcal{H}=\left\{(m, y) \in \mathbb{Z}^{2}, 0 \leq m \leq y\right\}$. The transition probabilities (tr) are given by:

$$
\begin{aligned}
& m=y \Rightarrow\left\{\begin{array}{l}
\operatorname{tr}((m, y),(m+1, y+1))=p \\
\operatorname{tr}((m, y),(m, y+1))=q
\end{array}\right. \\
& m<y \Rightarrow\left\{\begin{array}{l}
\operatorname{tr}((m, y),(m, y+1))=q \\
\operatorname{tr}((m, y),(m, y-1))=p
\end{array}\right.
\end{aligned}
$$


Figure 1 may serve as an illustration.

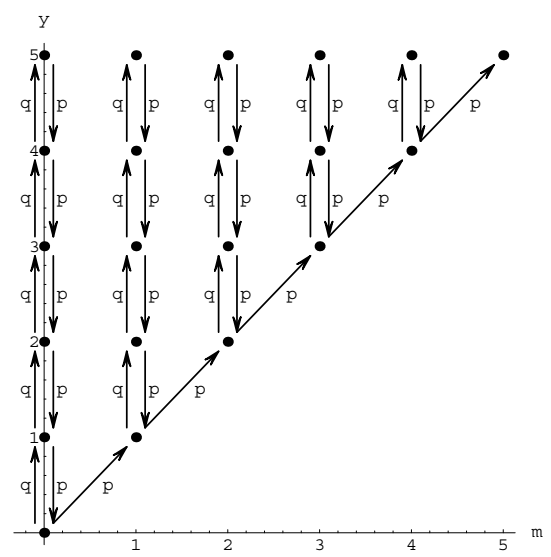

fig. 1: State space and transition probabilities of $\left(M_{n}, Y_{n}\right)_{n \geq 0}$

Proposition 2.3 Set $V_{a}=\inf \left\{n \geq 0: Y_{n}=a\right\}$, for $a \geq 0$. Then:

a) $V_{a}$ is a standard stopping time,

b) $V_{a}$ and $S_{V_{a}}$ are independent.

Remark 2.3 Again, by Proposition 2.1, it would suffice to consider the case $p=q=1 / 2$, although the following direct approach yields more quickly explicit formulas.

Proof. a) This is clear, since $\left|S_{n \wedge V_{a}}\right| \leq a$, for every $n$.

b) We will prove that $V_{a} \Perp M_{V_{a}}$, which is equivalent to the statement of the proposition, because $S_{V_{a}}=2 M_{V_{a}}-a$. Denote by $\Gamma_{j, k}^{a}$ the set of the paths of the process $\left(M_{n}, Y_{n}\right)$ leading from the origin to the point $\left(M_{V_{a}}=k, Y_{V_{a}}=a\right)$ in $V_{a}=a+2 j$ steps. The key observation is that the number of these paths, denoted by $\gamma_{j, k}^{a}$, does not depend on $k$. Indeed, as discussed in [10], the "projection" mapping:

$$
\Phi: \Gamma_{j, k}^{a} \rightarrow \Gamma_{j, 0}^{a},\left(M_{n}, Y_{n}\right)_{0 \leq n \leq a+2 j} \mapsto\left(0, Y_{n}\right)_{0 \leq n \leq a+2 j}
$$

is one-to-one, for every $k=0,1, \ldots, a$. As a result, we may write $\gamma_{j}^{a}$ instead of $\gamma_{j, k}^{a}$, for every $0 \leq k \leq a$.

We notice incidentally that $\gamma_{j}^{a}$ is the number of paths in $\mathbb{Z}$ which lead from 0 to $a-1$ in $a-1+2 j$ steps, staying in $\{0,1,2, \ldots, a-1\}$. By Lemma 2.3:

$$
\gamma_{j}^{a}=\alpha_{j}^{a-1,0}=\sum_{k \in \mathbb{Z}} C_{a-1+2 j}^{j+k(a+1)}-C_{a-1+2 j}^{j-1+k(a+1)} .
$$

The joint law of $\left(V_{a}, M_{V_{a}}\right)$ is given by

$$
\mathbb{P}\left(V_{a}=a+2 j \text { and } M_{V_{a}}=k\right)=\gamma_{j}^{a} p^{k+j} q^{a+j-k},
$$


whence the law of $V_{a}$ follows immediately:

$$
\begin{aligned}
\mathbb{P}\left(V_{a}=a+2 j\right) & =\gamma_{j}^{a} p^{j} q^{a+j} \sum_{k=0}^{a}\left(\frac{p}{q}\right)^{k} \\
& = \begin{cases}\gamma_{j}^{a} p^{j} q^{j} \frac{p^{a+1}-q^{a+1}}{p-q}, & \text { if } p \neq q, \\
\gamma_{j}^{a} \frac{a+1}{2^{a+2 j}}, & \text { if } p=q=\frac{1}{2} .\end{cases}
\end{aligned}
$$

In particular:

$$
\sum_{j=0}^{+\infty} \gamma_{j}^{a}(p q)^{j}=\frac{p-q}{p^{a+1}-q^{a+1}} \text { if } p \neq q \text { and } \sum_{j=0}^{+\infty} \frac{\gamma_{j}^{a}}{4^{j}}=\frac{2^{a}}{a+1} .
$$

From this, we derive the law of $M_{V_{a}}$ :

$$
\begin{aligned}
0 \leq k \leq a \Rightarrow \mathbb{P}( & \left.M_{V_{a}}=k\right)=p^{k} q^{a-k} \sum_{j=0}^{+\infty} \gamma_{j}^{a} p^{j} q^{j} \\
& = \begin{cases}\frac{p^{k} q^{a-k}(p-q)}{p^{a+1}-q^{a+1}}=r^{k} \frac{r-1}{r^{a+1}-1}, & \text { if } p \neq q, r:=\frac{p}{q}, \\
\left(\frac{1}{2}\right)^{a} \frac{2^{a}}{a+1}=\frac{1}{a+1}, & \text { if } p=q=\frac{1}{2} .\end{cases}
\end{aligned}
$$

Thus, for $p=q=1 / 2$, we have rediscovered the well-known fact that $M_{V_{a}}$ is uniformly distributed on $\{0,1, \ldots, a\}$. To complete the proof, it remains to check that $V_{a} \Perp M_{V_{a}}$, which is straightforward.

Corollary 2.2 Take $\varphi(z)=p e^{z}+q e^{-z}$ and denote by $\tilde{U}_{a}, a \geq 0$, the Chebyshev polynomials of the second kind.

(i) If $p \neq q$ then

$$
\psi_{V_{a}}(z)=\frac{(p-q) \sqrt{p^{a} q^{a}}}{p^{a+1}-q^{a+1}} \cdot \tilde{U}_{a}\left(\frac{\varphi(z)}{2 \sqrt{p q}}\right)
$$

and

$$
g_{V_{a}}(r)=\frac{p^{a+1}-q^{a+1}}{(p-q) \sqrt{p^{a} q^{a}}} \cdot \frac{1}{\tilde{U}_{a}\left(\frac{1}{2 \sqrt{p q} r}\right)} .
$$

(ii) If $p=q=1 / 2$ these equalities simplify to

$$
\tilde{\psi}_{V_{a}}(z)=\frac{\tilde{U}_{a}(\operatorname{ch} z)}{a+1}
$$

and

$$
\tilde{g}_{V_{a}}(r)=\frac{a+1}{\tilde{U}_{a}\left(\frac{1}{r}\right)}=(a+1) \sum_{j=0}^{+\infty} \gamma_{j}^{a}\left(\frac{r}{2}\right)^{a+2 j} .
$$

Proof. Similar to that of Corollary 2.1. 


\subsection{Example 3: The first hitting time of a given level by the age process.}

We denote by $g_{n}=\sup \left\{k \leq n: S_{k}=0\right\}$ the last return to zero before time $n$ and by $A_{n}=n-g_{n}$ the age of the walk at time $n$. Define

$$
\Lambda_{a}=\inf \left\{n \geq 0: A_{n}=a\right\}, \quad a \geq 0 .
$$

A surprising feature of this example is that $\Lambda_{a} \Perp S_{\Lambda_{a}}$ for any increment variable $X$. Note however that, if

$$
\tau:=\inf \left\{n>0: S_{n}=0\right\}=\infty \quad \text { a.s., }
$$

i.e. if the walk never returns to 0 , then $\Lambda_{a}=a$ a.s. and thus, $\Lambda_{a}$ is trivial. The next proposition shows that the joint law of $\left(S_{\Lambda_{a}}, \Lambda_{a}\right)$ is uniquely determined by that of $\left(S_{a}, \tau\right)$.

Proposition 2.4 For any $X$, we have:

$$
\mathbb{E}\left[\exp \left(\lambda S_{\Lambda_{a}}\right) r^{\Lambda_{a}}\right]=\frac{r^{a} \cdot \mathbb{E}\left[\exp \left(\lambda S_{a}\right) \mathbf{1}(\tau>a)\right]}{1-\sum_{k=1}^{a} \mathbb{P}(\tau=k) r^{k}} .
$$

Thus, defining

$$
H_{a}(z)=\frac{1}{\mathbb{P}(\tau>a)}\left[z^{a}-\sum_{k=0}^{a-1} \mathbb{P}(\tau=a-k) z^{k}\right],
$$

we get:

$$
g_{\Lambda_{a}}(r)=\frac{r^{a} \mathbb{P}(\tau>a)}{1-\sum_{k=1}^{a} \mathbb{P}(\tau=k) r^{k}}=\frac{1}{H_{a}\left(\frac{1}{r}\right)}
$$

and

$$
\psi_{\Lambda_{a}}(\lambda)=\mathbb{E}\left(\exp \left(\lambda S_{a}\right) / \tau>a\right)=H_{a}(\varphi(\lambda)),
$$

Moreover, $\Lambda_{a}$ is standard, and $\Lambda_{a} \Perp S_{\Lambda_{a}}$.

Remark 2.4 Statement d) of Theorem 1.1 holds in every case. Indeed, for any $X \neq 0$, the stopping time $\tau$ is unbounded, whence,

$$
|r| \leq 1 \Rightarrow \sum_{k=1}^{a} \mathbb{P}(\tau=k)|r|^{k} \leq \mathbb{P}(\tau \leq a)<1 .
$$

Thus, the first real root of the denominator in $(2.20)$, say $\rho$, is $>1 . \rho$ is also the radius of convergence of the generating function $g_{\Lambda_{a}}$.

Proof of Proposition 2.4. Set

$$
f(\lambda, r)=\mathbb{E}\left[\exp \left(\lambda S_{\Lambda_{a}}\right) r^{\Lambda_{a}}\right]
$$

and write $\left(\theta_{k}, k \geq 0\right)$ for the shift operators. 
By the strong Markov property and the fact that $\tau>a$ is equivalent to $\Lambda_{a}=a$, we get:

$$
\begin{array}{r}
f(\lambda, r)=\sum_{k=1}^{a} \mathbb{E}\left(\exp \left(\lambda S_{\Lambda_{a}}\right) r^{\Lambda_{a}} \mathbb{I}\{\tau=k\}\right)+\mathbb{E}\left(\exp \left(\lambda S_{\Lambda_{a}}\right) r^{\Lambda_{a}} \mathbb{I}\{\tau>a\}\right) \\
=\sum_{k=1}^{a} \mathbb{E}\left[\mathbb{E}\left(\exp \left(\lambda S_{\Lambda_{a} \circ \theta_{\tau}}\right) r^{\tau+\Lambda_{a} \circ \theta_{\tau}} \mathbb{I}\{\tau=k\} / \mathcal{F}_{\tau}\right)\right]+r^{a} \mathbb{E}\left(\exp \left(\lambda S_{a}\right) \mathbb{I}\{\tau>a\}\right) \\
=f(\lambda, r) \sum_{k=1}^{a} r^{k} \mathbb{P}(\tau=k)+r^{a} \mathbb{E}\left(\exp \left(\lambda S_{a}\right) \mathbb{I}\{\tau>a\}\right),
\end{array}
$$

whence (2.18) follows immediately. The rest of the proof is straightforward.

We now give explicit formulas in the case of a Bernoulli random walk.

Proposition 2.5 Under the assumption (2.1), the polynomial $H_{a}$ of Proposition 2.4 is given by

$$
H_{a}(z)=\tilde{H}_{a}\left(\frac{z}{2 \sqrt{p q}}\right) / \tilde{H}_{a}\left(\frac{1}{2 \sqrt{p q}}\right),
$$

where $\tilde{H}_{a}$ denotes this polynomial in the symmetric case. More precisely, for every $n \geq 0$,

$$
\begin{aligned}
\tilde{H}_{2 n}(z) & =\frac{1}{C_{2 n}^{n}}\left[(2 z)^{2 n}-\sum_{k=0}^{n-1} \frac{C_{2 n-2 k}^{n-k}}{(2 n-2 k-1)}(2 z)^{2 k}\right] \\
& =\sum_{k=0}^{n} \frac{\left(A_{n}^{k}\right)^{2}}{(1-2 n-2 k) A_{2 n}^{2 k}}(2 z)^{2 k}
\end{aligned}
$$

and

$$
\tilde{H}_{2 n+1}(z)=z \tilde{H}_{2 n}(z)
$$

Remark 2.5 It is easy to check the following recurrence relation between $\tilde{H}_{2 n}$ and $\tilde{H}_{2 n-1}$ :

$$
\tilde{H}_{2 n}(z)=\frac{2 n z \tilde{H}_{2 n-1}(z)-1}{2 n-1} .
$$

Corollary 2.3 The probability distribution of the first return to 0 in case of the general Bernoulli random walk is given by:

$$
\begin{aligned}
& \mathbb{P}(\tau=2 n)=\frac{1}{2 n-1} C_{2 n}^{n}(p q)^{n} \\
& \mathbb{P}(\tau>2 n)=\mathbb{P}(\tau>2 n+1)=C_{2 n}^{n}(p q)^{n} \tilde{H}_{2 n}\left(\frac{1}{2 \sqrt{p q}}\right) .
\end{aligned}
$$


Remark 2.6 (2.25) follows immediately from (4.14), page 352 in [4]. (2.26) seems to be less classical.

Proof of Proposition 2.5 and Corollary 2.3. The law of $\tau$ under $\tilde{\mathbb{P}}$ is classical (cf. [4], pp. 76-78):

$$
\tilde{\mathbb{P}}(\tau=2 k)=\frac{1}{2 k-1} C_{2 k}^{k} 2^{-2 k}
$$

and

$$
\tilde{\mathbb{P}}(\tau>2 k)=\tilde{\mathbb{P}}(\tau>2 k+1)=C_{2 k}^{k} 2^{-2 k} .
$$

Substituting both formulas in (2.19) yields (2.23). The recurrence relation (2.24) follows immediately from the fact that $\Lambda_{2 n+1}=\Lambda_{2 n}+1$, a.s. Comparing (2.21) and (2.8), we get (2.22), whence the corollary can be easily derived.

\subsection{Example 4: A randomized stopping time solving (IP)}

Here again, we do not need to specify $X$, but we assume that the filtration $\left(\mathcal{F}_{n}, n \geq 0\right)$ is natural. Let $T$ be a non-zero $\left(\mathcal{F}_{n}\right)$-stopping time solving (IP). Define inductively an increasing sequence of stopping times $\left(T_{n}, n \geq 0\right)$ :

$$
T_{0}=0 \text { and } T_{n+1}= \begin{cases}T_{n}+T \circ \theta_{T_{n}}, & \text { if } S_{T_{n}}=0, \\ T_{n}, & \text { otherwise }\end{cases}
$$

where the $\left(\theta_{j}, j \geq 0\right)$ are again the shift operators.

Now, in order to augment the filtration $\left(\mathcal{F}_{n}, n \geq 0\right)$, let $\left(Y_{n}, n \geq 1\right)$ be a sequence of i.i.d. random variables having the Bernoulli distribution with parameter $\rho, 0 \leq \rho \leq 1$. Assume that the $\sigma$-algebras $\mathcal{Y}_{\infty}=\sigma\left(Y_{n}, n \geq 1\right)$ and $\mathcal{F}_{\infty}=\vee_{k=0}^{\infty} \mathcal{F}_{k}$ are independent. Putting

$$
\hat{\mathcal{F}}_{n}=\mathcal{F}_{n} \vee \mathcal{Y}_{\infty}, n \geq 0,
$$

we define a $\left(\hat{\mathcal{F}}_{n}\right)$-stopping time $\hat{T}$ solving (IP) as follows:

$$
\hat{T}=T_{n} \Leftrightarrow\left\{\begin{array} { c } 
{ S _ { T _ { 1 } } = 0 } \\
{ Y _ { 1 } = 0 }
\end{array} \text { and } \ldots \text { and } \left\{\begin{array} { c } 
{ S _ { T _ { n - 1 } } = 0 } \\
{ Y _ { n - 1 } = 0 }
\end{array} \text { and } \left\{\begin{array}{c}
S_{T_{n}} \neq 0 \\
\text { or } Y_{n}=1 .
\end{array}\right.\right.\right.
$$

Thus $\hat{T}$ is the first time $T_{n}$ such that $S_{T_{n}} \neq 0$ or $Y_{n}=1$. In both special cases $\rho=0$ and $\rho=1$, the $\sigma$-algebra $\mathcal{Y}_{\infty}$ is trivial, so that $\hat{T}$ is actually a stopping time in the natural filtration. In particular, if $\rho=1$ or $\mathbb{P}\left(S_{T}=0\right)=0$, then $\hat{T}=T$ a.s. 
Proposition 2.6 Under the above assumptions, $\hat{T}$ satisfies (IP). Moreover, setting $\alpha=\mathbb{P}\left(S_{T}=0\right)$ and $\sigma=\mathbb{P}\left(Y_{1}=0\right)=1-\rho$, we have:

$$
\psi_{\hat{T}}(z)=\frac{\psi_{T}(z)-\alpha \sigma}{1-\alpha \sigma}
$$

and

$$
g_{\hat{T}}(r)=\frac{(1-\alpha \sigma) g_{T}(r)}{1-\alpha \sigma g_{T}(r)}
$$

Remark 2.7 (i) The intuitive purpose of the parameter $\sigma$ is to remove an arbitrary amount of mass from 0 in the distribution of $S_{T}$. In the special case $\sigma=1$, the mass on 0 is totally removed, so that $\mathcal{L}\left(S_{\hat{T}}\right)=\mathcal{L}\left(S_{T} / S_{T} \neq 0\right)$. When $\sigma=0$, the weight of 0 is left unchanged, so that $\mathcal{L}\left(S_{\hat{T}}\right)=\mathcal{L}\left(S_{T}\right)$.

(ii) The stopping time $\hat{T}$ is randomized. In case of the symmetric Bernoulli random walk, it is possible to choose the variables $\left(Y_{n}, n \geq 1\right)$ in such a way that each $Y_{n}$ is $\mathcal{F}_{T_{n}}$-measurable. $\hat{T}$ then becomes a stopping time in the natural filtration (cf. Proposition 3.3).

Proof of Proposition 2.6. Set $\mathcal{N}=\left\{S_{T}=0\right\}$. $\hat{T}$ satisfies the following identity in law:

$$
\hat{T} \stackrel{d}{=} T \mathbb{1}_{\left[\mathcal{N}^{c} \cup(\mathcal{N} \cap\{Y=1\})\right]}+\left(T+\hat{T} \circ \theta_{T}\right) \mathbb{1}_{[\mathcal{N} \cap\{Y=0\}]} .
$$

Thus, to establish the joint distribution of $\left(S_{\hat{T}}, \hat{T}\right)$, we compute:

$$
\begin{aligned}
\widehat{f}(\lambda, r):=\mathbb{E} & {\left[\exp \left(\lambda S_{\widehat{T}}\right) r^{\widehat{T}}\right] } \\
=\mathbb{E} & {\left[\exp \left(\lambda S_{T}\right) r^{T} \mathbb{1}_{\left[\mathcal{N}^{c} \cup(\mathcal{N} \cap\{Y=1\})\right]}\right] } \\
& +\mathbb{E}\left[\exp \left(\lambda S_{T+\widehat{T} \circ \theta_{T}}\right) r^{T+\widehat{T} \circ \theta_{T}} \mathbb{I}_{[\mathcal{N} \cap\{Y=0\}]}\right] .
\end{aligned}
$$

The first term is

$$
\begin{aligned}
& \mathbb{E}\left[\exp \left(\lambda S_{T}\right) r^{T} \mathbb{1}_{[\mathcal{N} c \cup(\mathcal{N} \cap\{Y=1\})]}\right]=\mathbb{E}\left[\exp \left(\lambda S_{T}\right) r^{T}\left(\mathbb{I}_{\left\{S_{T} \neq 0\right\}}+\mathbb{I}_{\left\{S_{T}=0\right\}} \mathbb{I}_{\{Y=1\}}\right)\right] \\
& =\mathbb{E}\left[\exp \left(\lambda S_{T}\right) r^{T}\left(1-\mathbb{I}_{\left\{S_{T}=0\right\}} \mathbb{I}_{\{Y=0\}}\right)\right] .
\end{aligned}
$$

Applying the Markov property to the second term, we get

$$
\begin{aligned}
& \mathbb{E}\left[\exp \left(\lambda S_{T+\widehat{T} \circ \theta_{T}}\right) r^{T+\widehat{T} \circ \theta_{T}} \mathbb{I}_{[\mathcal{N} \cap\{Y=0\}]}\right] \\
& =\mathbb{E}\left[r^{T} \mathbb{I}_{\{Y=0\}} \mathbb{I}_{\mathcal{N}} \mathbb{E}\left(\exp \left(\lambda\left(S_{T+\widehat{T} \circ \theta_{T}}-S_{T}\right)\right) r^{\widehat{T} \circ \theta_{T}} / \widehat{\mathcal{F}}_{T}\right)\right] \\
& =\widehat{f}(\lambda, r) \mathbb{E}\left[r^{T} \mathbb{I}_{\{Y=0\}} \mathbb{I}_{\mathcal{N}}\right] .
\end{aligned}
$$

By the independence assumption, we have

$$
\mathbb{E}\left[r^{T} \mathbb{I}_{\{Y=0\}} \mathbb{I}_{\mathcal{N}}\right]=\sigma \alpha g_{T}(r),
$$

where $\sigma=\mathbb{P}\left(Y_{1}=0\right)$ and $\alpha=\mathbb{P}(\mathcal{N})$. 
Substituting everything in (2.32) yields

$$
\begin{aligned}
\widehat{f}(\lambda, r) & =\frac{\mathbb{E}\left[\exp \left(\lambda S_{T}\right) r^{T}\left(1-\mathbb{I}_{\left\{S_{T}=0\right\}} \mathbb{I}_{\{Y=0\}}\right)\right]}{1-\sigma \alpha g_{T}(r)} \\
& =\frac{\left(\psi_{T}(\lambda)-\sigma \alpha\right) g_{T}(r)}{1-\sigma \alpha g_{T}(r)} .
\end{aligned}
$$

The rest of the proof is standard.

Remark 2.8 When $T$ is a constant stopping time, say $T=N$ a.s., we have

$$
\psi_{\hat{T}}(z)=\frac{\varphi(z)^{N}-\alpha \sigma}{1-\alpha \sigma} \quad \text { and } \quad g_{\hat{T}}(r)=\frac{(1-\alpha \sigma) r^{N}}{1-\alpha \sigma r^{N}} .
$$

\subsection{Examples obtained by iteration}

Throughout this paragraph, we will assume that $\left(\mathcal{F}_{n}, n \geq 0\right)$ is the natural filtration of the walk. We give a simple method which yields a large class of stopping times solving (IP).

Let $T_{1}$ be a $\left(\mathcal{F}_{n}\right)$-stopping time satisfying (IP). Then $\left(S_{n}^{\prime}:=S_{T_{1}+n}-\right.$ $\left.S_{T_{1}}, n \geq 0\right)$ is a random walk independent of $\mathcal{F}_{T_{1}}$ and with the same distribution as $\left(S_{n}, n \geq 0\right)$; denote by $\left(\mathcal{F}_{n}^{\prime}, n \geq 0\right)$ its natural filtration. Let $T_{2}$ be a $\left(\mathcal{F}_{n}^{\prime}\right)$-stopping time, also satisfying (IP). Then $T=T_{1}+T_{2}$ is a standard stopping time such that $T \Perp S_{T}$. Indeed, $\left(T_{2}, S_{T_{2}}^{\prime}\right)$ and $\left(T_{1}, S_{T_{1}}\right)$ are independent and the components of each pair are independent by hypothesis. As a result, the four random variables $T_{1}, S_{T_{1}}, T_{2}$ and $S_{T_{2}}^{\prime}$ are independent, whence the independence of $T=T_{1}+T_{2}$ and $S_{T}=S_{T_{1}}+S_{T_{2}}^{\prime}$ follows. Clearly, $T$ is standard since for instance, $\mathbb{E} T=\mathbb{E} T_{1}+\mathbb{E} T_{2}<+\infty$. Iterating the above construction leads to the following

Proposition 2.7 Given a finite sequence $\left(T_{1}, \ldots, T_{k}\right)$ of $\left(\mathcal{F}_{n}\right)$-stopping times solving $(I P)$, it is possible to construct another $\left(\mathcal{F}_{n}\right)$-stopping time $T$ solving (IP), such that

$$
\begin{aligned}
\mathcal{L}(T) & =\mathcal{L}\left(T_{1}\right) * \ldots * \mathcal{L}\left(T_{k}\right) \\
\mathcal{L}\left(S_{T}\right) & =\mathcal{L}\left(S_{T_{1}}\right) * \ldots * \mathcal{L}\left(S_{T_{k}}\right) .
\end{aligned}
$$

To illustrate the iteration method, we take again the symmetric Bernoulli random walk. For any sequence $\left(a_{k}\right)_{1 \leq k \leq n}$ of positive integers, we define a stopping time $T_{a_{1}, \ldots, a_{n}}^{*}$ by the following recurrence relation:

$$
T_{a_{1}, \ldots, a_{n}}^{*}=T_{a_{1}, \ldots, a_{n-1}}^{*}+T_{a_{n}}^{*} \circ \theta_{T_{a_{1}, \ldots, a_{n-1}}^{*}},
$$

where $T_{a_{1}}^{*}$ is the stopping time of Example 1 . 
By Proposition 2.7 and Corollary 2.1, we have $\mathbb{E}\left(\exp z S_{T_{a_{1}, \ldots, a_{n}}^{*}}\right)=\prod_{k=1}^{n} \operatorname{ch}\left(a_{k} z\right)=\frac{1}{2^{n}} \sum_{\left(\varepsilon_{1}, \ldots, \varepsilon_{n}\right) \in\{-1,1\}^{n}} \exp \left[\left(\varepsilon_{1} a_{1}+\ldots+\varepsilon_{n} a_{n}\right) z\right]$. In other words,

$$
\mathcal{L}\left(S_{T_{a_{1}, \ldots, a_{n}}^{*}}\right)=\mathcal{L}\left(a_{1} \varepsilon_{1}+\ldots+a_{n} \varepsilon_{n}\right)
$$

where the $\varepsilon_{k}$ are i.i.d. random variables with $\mathbb{P}\left(\varepsilon_{k}= \pm 1\right)=1 / 2$. Permuting the $\left(a_{k}\right)_{1 \leq k \leq n}$ does not change this probability distribution, denoted simply by $\mathcal{L}\left(a_{1}, \ldots, a_{n}\right)$. Thus, stopping times $T$ satisfying (IP) and such that $S_{T}$ is distributed according to $\mathcal{L}\left(a_{1}, \ldots, a_{n}\right)$ are in general not unique.

To conclude, we note that the generating function of $T_{a_{1}, \ldots, a_{n}}^{*}$ is given by

$$
\mathbb{E}\left(r^{T_{a_{1}, \ldots, a_{n}}^{*}}\right)=\prod_{k=1}^{n} \frac{1}{\tilde{C}_{a_{k}}\left(\frac{1}{r}\right)},
$$

where $\tilde{C}_{a}$ is again the Chebyshev polynomial of first kind and degree $a$.

\section{Stopping distributions in the Bernoulli case}

Throughout this section, we will assume that $S$ is the symmetric Bernoulli random walk and that the filtration $\left(\mathcal{F}_{n}, n \geq 0\right)$ is natural. Our goal is to describe all possible distributions of $S_{T}$, where $T$ runs through the set $\mathcal{T}$ of all $\left(\mathcal{F}_{n}\right)$-stopping times solving (IP). In the sequel, these distributions will simply be called "stopping distributions".

\subsection{Definition and characterization}

Let $T \in \mathcal{T}$ and $m=\min T$. Recall from Lemma 2.1 that

$$
\begin{aligned}
& \text { Supp } \mathcal{L}\left(S_{T}\right) \subset\{m-2 j, 0 \leq j \leq m\} \text { and } \\
& \text { Supp } \mathcal{L}(T) \subset\{m+2 k, k \geq 0\} .
\end{aligned}
$$

Here and in all that follows, we use the notation

$$
p_{j}:=\mathbb{P}\left(S_{T}=m-2 j\right), 0 \leq j \leq m .
$$

These probabilities have a particular form. Indeed, since the filtration is assumed to be natural, the event $\left\{S_{T}=m-2 j, T=m\right\}$ depends only on the history of the walk until time $m$. Among the $C_{m}^{j}$ paths leading from the origin to $M_{j}(m, m-2 j)$, write $\pi_{j}$ for the number of paths stopped at time $m$. 
Thus,

$$
\mathbb{P}\left(S_{T}=m-2 j \text { and } T=m\right)=\pi_{j} 2^{-m}
$$

and summing this expression over $j$, we get

$$
\mathbb{P}(T=m)=\sum_{j=0}^{m} \pi_{j} 2^{-m} .
$$

The independence assumption yields

$$
\left(\mathrm{N}_{1}\right) \quad p_{j}=\frac{\pi_{j}}{\sum_{l=0}^{m} \pi_{l}}, \text { where } \pi_{j} \in \mathbb{N} \text { and } 0 \leq \pi_{j} \leq C_{m}^{j}, \forall j \in\{0,1, \ldots, m\} .
$$

The symmetry of $\mathcal{L}\left(S_{T}\right)$ translates into

$$
\pi_{j}=\pi_{m-j}, \forall j \in\{0,1, \ldots, m\},
$$

Moreover,

$$
\pi_{0}=\pi_{m}=1,
$$

since the only path leading from the origin to the point $M_{0}$ has to be stopped. Notice finally that the number of paths going through $M_{j}$ and being stopped after time $m$ is given by

$$
\theta_{j}:=C_{m}^{j}-\pi_{j}
$$

where in particular $\theta_{0}=\theta_{m}=0$.

More generally, for $k \geq 0$ and $0 \leq j \leq m$, we denote by $\pi_{j, k}\left(\right.$ resp. $\left.\theta_{j, k}\right)$ the number of paths leading from the origin to the point $M_{j, k}(m+2 k, m-2 j)$ and being stopped at (resp. after) time $m+2 k$. As above, we have

$$
\mathbb{P}\left(S_{T}=m-2 j \text { and } T=m+2 k\right)=\pi_{j, k} 2^{-m-2 k}
$$

and

$$
\mathbb{P}(T=m+2 k)=\sum_{j=0}^{m} \pi_{j, k} 2^{-m-2 k} .
$$

By the independence assumption,

$$
p_{j}=\frac{\pi_{j, k}}{\sum_{l=0}^{m} \pi_{l, k}}, 0 \leq j \leq m, k \geq 0 .
$$

Thus, the total number of paths stopped at or going through $M_{j, k}$ is given by

$$
\nu_{j, k}=\pi_{j, k}+\theta_{j, k},
$$

or alternatively,

$$
\nu_{j, k}=\theta_{j-1, k-1}+2 \theta_{j, k-1}+\theta_{j+1, k-1} .
$$


This equality remains valid for $j=0$ and $j=m$, provided that we set $\theta_{-1, k}=\theta_{m+1, k}=0$. Obviously $\theta_{0, k}=\theta_{m, k}=0$, otherwise the walk would leave $\llbracket-m, m \rrbracket$ with positive probability. On the other hand, we have

$$
\pi_{j, k}=\pi_{j} \pi_{0, k}=\pi_{j} \theta_{1, k-1} .
$$

Indeed, the first equality is a consequence of the independence assumption whereas the second one comes from the fact that the $\theta_{1, k-1}$ trajectories going through $M_{1, k-1}$ without being stopped have in particular $\theta_{1, k-1}$ connections to $M_{0, k}$, which have to be stopped.

Equalities (3.7), (3.8) and (3.9) now easily lead to the following recurrence system:

$$
\left\{\begin{array}{l}
\theta_{j, k}=\theta_{j-1, k-1}+2 \theta_{j, k-1}+\theta_{j+1, k-1}-\pi_{j} \theta_{1, k-1} \\
\theta_{j, 0}=\theta_{j}
\end{array}, 0 \leq j \leq m, k \geq 1\right.
$$

which can be put into matrix form:

$$
\Theta_{k}=\Pi^{k} \Theta_{0}, k \geq 0
$$

where $\Theta_{0}=\left(\theta_{0}, \theta_{1}, \ldots, \theta_{m}\right)^{\mathrm{t}}, \Theta_{k}=\left(\theta_{0, k}, \theta_{1, k}, \ldots, \theta_{m, k}\right)^{\mathrm{t}}$ and

$$
\Pi=\left(\begin{array}{ccccccc}
2 & 1-\pi_{0} & 0 & \ldots & \ldots & \ldots & 0 \\
1 & 2-\pi_{1} & 1 & 0 & \ldots & \ldots & \ldots \\
0 & 1-\pi_{2} & 2 & 1 & 0 & \ldots & \ldots \\
0 & -\pi_{3} & 1 & 2 & 1 & 0 & \ldots \\
\ldots & \ldots & \ldots & \ldots & \ldots & \ldots & \ldots \\
0 & -\pi_{m-1} & \ldots & 0 & 1 & 2 & 1 \\
0 & -\pi_{m} & \ldots & \ldots & 0 & 1 & 2
\end{array}\right)
$$

Furthermore, since $\theta_{j}=\theta_{m-j}$ and $\theta_{0}=\theta_{m}=0$, we have:

$$
\theta_{j, k}=\theta_{m-j, k} \text { and } \theta_{0, k}=\theta_{m, k}=0,0 \leq j \leq m, k \geq 0 .
$$

The coefficients $\pi_{j, k}$ and $\nu_{j, k}$ can now be derived from the $\theta_{j, k}$ via (3.9) and (3.7). Note that every $\theta_{j, k}$ is necessarily $\geq 0$, since $\pi_{j, k}$ must be $\leq \nu_{j, k}$.

For the converse, start with some sequence $\left(\pi_{j}, 0 \leq j \leq m\right)$, satisfying the necessary conditions $\left(\mathrm{N}_{1}\right)$ to $\left(\mathrm{N}_{3}\right)$ and compute the $\theta_{j}$ and the $\theta_{j, k}$ according to (3.3) and (3.10), respectively. If $\theta_{j, k} \geq 0$, for any $j$ and any $k$, then it is possible to construct a stopping time $T \in \mathcal{T}$ such that $\mathcal{L}\left(S_{T}\right)=\left\{\left(m-2 j, p_{j}\right), 0 \leq j \leq m\right\}$. This is achieved by choosing deterministically for each path $\omega$ the corresponding stopping time $T(\omega)$, in such a way that the recurrence system (3.10) is fulfilled. $T$ is then trivially standard since, by construction, $\left|S_{n \wedge T}\right| \leq m$, for every $n$. Thus we have the following 
Proposition 3.1 Assume that $S$ is the symmetric Bernoulli random walk and that the filtration $\left(\mathcal{F}_{n}, n \geq 0\right)$ is natural. Let

$$
\mathcal{L}=\left\{\left(m-2 j, p_{j}\right) / 0 \leq j \leq m\right\}
$$

be a probability distribution satisfying conditions $\left(N_{1}\right)$ to $\left(N_{3}\right)$. Then the following are equivalent:

(i) $\mathcal{L}$ is a stopping distribution,

(ii) $\forall j \in\{0, \ldots, m\}, \forall k \in \mathbb{N}, \theta_{j, k} \geq 0$, where the $\theta_{j, k}$ satisfy (3.10) with the $\theta_{j}$ given by (3.3) .

Remark 3.1 It seems difficult to list all stopping distributions for a given $m=\min T$. To simplify the task, one may begin by discarding distributions that are inadmissible a priori. To be precise, given a random variable $U$ with a distribution $\mathcal{L}=\left\{\left(m-2 j, p_{j}\right), 0 \leq j \leq m\right\}$ satisfying conditions $\left(\mathrm{N}_{1}\right)$ to $\left(\mathrm{N}_{3}\right)$, consider the power series expansion of $1 / \tilde{P}_{m}(1 / r)$ where $\tilde{P}_{m}$ is defined by $\mathbb{E}(\exp z U)=\tilde{P}_{m}(\operatorname{ch} z)$; whenever $\mathcal{L}$ is a stopping distribution, Wald's identity entails that all the coefficients of this power series are nonnegative. In other words, if some of the coefficients are negative, then $\mathcal{L}$ cannot be a stopping distribution. This strategy will be applied successfully to settle the cases $m=1,2,3$ and 4 .

Now, for any $T \in \mathcal{T}$ with minimum $m$, consider the polynomial $\tilde{P}_{m}$ introduced in Lemma 2.2. We have:

$$
\tilde{P}_{m}(\operatorname{ch} \lambda)=\mathbb{E}\left(\operatorname{ch} \lambda S_{T}\right)=\frac{1}{\sum_{j=0}^{m} \pi_{j}} \sum_{j=0}^{m} \pi_{j} \operatorname{ch}(\lambda(m-2 j)) .
$$

Recalling that $\tilde{C}_{k}$ denotes the $k^{\text {st }}$ Chebyshev polynomial of first kind and setting for convenience $\tilde{C}_{-k}=\tilde{C}_{k}, k \geq 0$, we may write equivalently

$$
\tilde{P}_{m}(r)=\frac{1}{\sum_{j=0}^{m} \pi_{j}} \sum_{j=0}^{m} \pi_{j} \tilde{C}_{m-2 j}(r) .
$$

The distribution of $T$ can be expressed in terms of the $\pi_{j}$ and $\theta_{1, k}$. Indeed, with the convention that $\theta_{1,-1}=1,(3.5)$ and (3.9) yield

$$
\mathbb{P}(T=m+2 l)=\left(\sum_{j=0}^{m} \pi_{j}\right) \theta_{1, l-1} 2^{-m-2 l}, l \geq 0 .
$$

Hence,

$$
g_{T}(r)=\left(\sum_{j=0}^{m} \pi_{j}\right) \sum_{l=0}^{+\infty} \theta_{1, l-1}\left(\frac{r}{2}\right)^{m+2 l}
$$


Substituting (3.13) and (3.14) in Wald's identity, we get:

$$
\sum_{j=0}^{m} \pi_{j} \tilde{C}_{m-2 j}(r) \cdot \sum_{l=0}^{+\infty} \theta_{1, l-1}(2 r)^{-m-2 l}=1
$$

Let us go further into the study of the coefficients $\theta_{j, k}$. First we observe that

$$
\theta_{j, k} 2^{-m-2 k}=\mathbb{P}\left(S_{m+2 k}=m-2 j \text { and } T>m+2 k\right) .
$$

Unfortunately, the right-hand side in this equality does not depend directly on the joint distribution of $\left(S_{T}, T\right)$. To remedy this defect, we proceed as follows:

$$
\sum_{j=0}^{m} \theta_{j, k} 2^{-m-2 k} \exp [\lambda(m-2 j)]=\mathbb{E}\left[\exp \left(\lambda S_{m+2 k}\right) \mathbb{I}\{T>m+2 k\}\right] .
$$

On the other hand, by the martingale property, we have, for every $n \geq 0$,

$$
\begin{aligned}
& \mathbb{E}\left[\exp \left(\lambda S_{n \wedge T}\right)(\operatorname{ch} \lambda)^{-n \wedge T}\right]=1=\mathbb{E}\left[\exp \left(\lambda S_{T}\right)(\operatorname{ch} \lambda)^{-T}\right] \\
& \Longleftrightarrow \mathbb{E}\left[\exp \left(\lambda S_{n}\right)(\operatorname{ch} \lambda)^{-n} \mathbb{I}\{T>n\}\right]+\mathbb{E}\left[\exp \left(\lambda S_{T}\right)(\operatorname{ch} \lambda)^{-T} \mathbb{I}\{T \leq n\}\right] \\
& =\mathbb{E}\left[\exp \left(\lambda S_{T}\right)(\operatorname{ch} \lambda)^{-T}\right] \\
& \Longleftrightarrow \mathbb{E}\left[\exp \left(\lambda S_{n}\right)(\operatorname{ch} \lambda)^{-n} \mathbb{I}\{T>n\}\right]=\mathbb{E}\left[\exp \left(\lambda S_{T}\right)\right] \mathbb{E}\left[(\operatorname{ch} \lambda)^{-T} \mathbb{I}\{T>n\}\right] .
\end{aligned}
$$

Thus,

$$
\begin{aligned}
\mathbb{E}\left[\exp \left(\lambda S_{n}\right) \mathbb{I}\{T>n\}\right] & =(\operatorname{ch} \lambda)^{n} \mathbb{E}\left[\exp \left(\lambda S_{T}\right)\right] \mathbb{E}\left[(\operatorname{ch} \lambda)^{-T} \mathbb{I}\{T>n\}\right] \\
& =(\operatorname{ch} \lambda)^{n} \tilde{P}_{m}(\operatorname{ch} \lambda) \mathbb{E}\left[(\operatorname{ch} \lambda)^{-T} \mathbb{I}\{T>n\}\right],
\end{aligned}
$$

whence it is natural to introduce

$$
\begin{aligned}
& Q_{k}(r)=(2 r)^{m+2 k} \tilde{P}_{m}(r) \mathbb{E}\left[r^{-T} \mathbb{I}\{T>m+2 k\}\right] \\
& =(2 r)^{2 k} \sum_{j=0}^{m} \pi_{j} \tilde{C}_{m-2 j}(r) \cdot \sum_{l>k} \theta_{1, l-1}(2 r)^{-2 l}=(2 r)^{m+2 k} \frac{\sum_{l>k} \theta_{1, l-1}(2 r)^{-2 l}}{\sum_{l=0}^{+\infty} \theta_{1, l-1}(2 r)^{-2 l}} .
\end{aligned}
$$

Note incidentally that the last equality follows immediately from (3.15). Combining (3.16) and (3.17), we now see that:

$$
\sum_{j=0}^{m} \theta_{j, k} \exp [\lambda(m-2 j)]=Q_{k}(\operatorname{ch} \lambda)
$$

or alternatively, using symmetry,

$$
\sum_{j=0}^{m} \theta_{j, k} \tilde{C}_{m-2 j}(\operatorname{ch} \lambda)=Q_{k}(\operatorname{ch} \lambda) .
$$


Substituting $\operatorname{ch} \lambda=r$ in (3.19), we see that $Q_{k}(r)$ is, for every $k \geq 0$, a polynomial of degree at most $m-2$ (remember that $\left.\theta_{0, k}=\theta_{m, k}=0\right)$. The coefficients $\theta_{j, k}$ in the decomposition

$$
Q_{k}(r)=\sum_{j=0}^{m} \theta_{j, k} \tilde{C}_{m-2 j}(r)
$$

are nonnegative integers. They are unique if we impose the additional condition that $\tilde{C}_{n}$ and $\tilde{C}_{-n}$ have the same coefficient.

Substituting $e^{-\lambda}=r$ in (3.18), we obtain similarly that

$$
\sum_{j=0}^{m} \theta_{j, k} r^{2 j}=r^{m} Q_{k}\left(\frac{r^{2}+1}{2 r}\right)
$$

which is, for every $k \geq 0$, an even polynomial of degree at most $2 m-2$, whose coefficients are nonnegative integers and symmetric (i.e. $\theta_{j, k}=\theta_{m-j, k}$ ).

The recurrence system (3.10) and equality (3.20) have been established by combinatorial arguments. They can also be derived in a purely algebraic way from Wald's identity and some natural hypotheses on the support of the distributions of $T$ and $S_{T}$, as will be shown in the following proposition. We will see furthermore that the distribution of $T$ has a very special form.

Proposition 3.2 Let $U$ and $V$ be two integer-valued independent random variables such that, for some positive integer $m$,

$$
\begin{aligned}
& \mathcal{L}(U)=\left\{\left(m-2 j, p_{j}\right) / 0 \leq j \leq m\right\}, \text { where the } p_{j} \text { satisfy }\left(N_{1}\right) \text { to }\left(N_{3}\right), \\
& \mathcal{L}(V)=\left\{\left(m+2 k, q_{k}\right) / k \geq 0\right\} \text { where } q_{0} \neq 0,
\end{aligned}
$$

and

$$
\mathbb{E}(\exp \lambda U) \mathbb{E}\left((\operatorname{ch} \lambda)^{-V}\right)=1, \forall \lambda \in \mathbb{R}
$$

a) There exist unique integers $\mu$ and $\hat{b}_{0}, \hat{b}_{1}, \ldots, \hat{b}_{\mu}$, with $\hat{b}_{0}=1, \hat{b}_{\mu} \neq 0$ and $2 \mu \leq m$, such that:

$$
\pi_{j}=\sum_{l=0}^{\mu} \hat{b}_{l} C_{m-2 l}^{j-l} \leq j \leq m .
$$

b) There exist unique integers $\left(\hat{q}_{k}, k \geq 0\right)$ with $\hat{q}_{0}=1$, such that

$$
\hat{q}_{k}=\left(\sum_{j=0}^{m} \pi_{j}\right) \hat{q}_{k} 2^{-m-2 k}=\left(\sum_{l=0}^{\mu} \hat{b}_{l} 2^{-2 l}\right) \hat{q}_{k} 2^{-2 k}, \quad k \geq 0 .
$$


c) Moreover, the $\left(\hat{q}_{k}, k \geq 0\right)$ are uniquely determined by the following system:

$$
\begin{cases}\hat{b}_{0} \hat{q}_{0}=1 & \left(E_{0}\right) \\ \sum_{l=0}^{\mu \wedge k} \hat{b}_{l} \hat{q}_{k-l}=0 & \left(E_{k}\right), k \geq 1\end{cases}
$$

d) Let $\hat{B}$ be the "generating polynomial" of $U$, defined by

$$
\hat{B}(z)=\sum_{i=0}^{\mu} \hat{b}_{i} z^{\mu-i} .
$$

The roots of $\hat{B}$, denoted by $z_{1}, \ldots, z_{w}$, with respective multiplicity $m_{1}, \ldots, m_{w}$, are all of modulus $<4$.

There exist unique complex constants $\left(c_{s, r}\right)_{1 \leq s \leq w, 0 \leq r \leq m_{s}-1}$ such that

$$
\hat{q}_{k}=\sum_{s=1}^{w} \sum_{r=0}^{m_{s}-1} c_{s, r} k^{r} z_{s}^{k}, \quad k \geq n .
$$

e) For every $k \geq 0$,

$$
Q_{k}(r)=(2 r)^{m+2 k} \frac{\sum_{i>k} q_{i} r^{-2 i}}{\sum_{i=0}^{+\infty} q_{i} r^{-2 i}}
$$

is a polynomial with integer coefficients, of degree at most $m-2$. It is expressed in terms of the Chebyshev polynomials of first kind as follows:

$$
Q_{k}(r)=\sum_{j=0}^{m} \hat{\theta}_{j, k} \tilde{C}_{m-2 j}(r),
$$

where the $\hat{\theta}_{j, k}$ are integers, and more precisely:

$$
\hat{\theta}_{j, k}=\sum_{h=1}^{\mu} C_{m-2 h}^{j-h} \sum_{i=0}^{h-1} \hat{b}_{i} \hat{q}_{k+h-i} .
$$

In particular, $\hat{\theta}_{j, k}=\hat{\theta}_{m-j, k}, \hat{\theta}_{0, k}=0$ and $\hat{\theta}_{1, k}=\hat{q}_{k+1}$.

f) For every $k \geq 0$,

$$
\hat{Q}_{k}(r)=r^{m} Q_{k}\left(\frac{r^{2}+1}{2 r}\right)=\sum_{j=1}^{m-1} \hat{\theta}_{j, k} r^{2 j}
$$

is a polynomial of degree at most $2 m-2$, with symmetric integer coefficients.

g) The recurrence system (3.10) remains valid:

$$
\left\{\begin{array}{l}
\hat{\theta}_{j, k}=\hat{\theta}_{j-1, k-1}+2 \hat{\theta}_{j, k-1}+\hat{\theta}_{j+1, k-1}-\pi_{j} \hat{\theta}_{1, k-1} \quad, 0 \leq j \leq m, k \geq 1 \\
\hat{\theta}_{j, 0}=C_{m}^{j}-\pi_{j}
\end{array}\right.
$$


Remark 3.2 (i) We stress the fact that the "generating polynomial" of $U$, as defined in the above proposition, does not suffice to determine $\mathcal{L}(U)$. However, in conjunction with $m$, the maximum of the support, it does. (ii) By Proposition $(3.1), \mathcal{L}(U)$ is a stopping distribution iff the $\hat{\theta}_{j, k}$ are all $\geq 0$.

Proof of Proposition 3.2. a) Consider again the polynomial $\tilde{P}_{m}$ defined by $\tilde{P}_{m}(\operatorname{ch} \lambda)=\mathbb{E}(\exp \lambda U)$, and expand it:

$$
\tilde{P}_{m}(z)=\sum_{j=0}^{m} p_{j} \tilde{C}_{m-2 j}(z)=\sum_{i=0}^{\mu} a_{i} z^{m-2 i},
$$

with the convention that $a_{\mu} \neq 0$, that is, $a_{\mu} z^{m-2 \mu}$ is the term of least degree, or equivalently, $m_{0}:=m-2 \mu \geq 0$ is the "multiplicity" of 0 . When multiplying both sides in the above equality by $\sum_{j=0}^{m} \pi_{j}$, we get a polynomial $\hat{P}_{m}$ with integer coefficients, which is more convenient for our purpose:

$$
\hat{P}_{m}(z)=\sum_{j=0}^{m} \pi_{j} \tilde{C}_{m-2 j}(z)=\sum_{i=0}^{\mu} \hat{a}_{i} z^{m-2 i},
$$

where $\hat{a}_{i}=a_{i} \sum_{j=0}^{m} \pi_{j}$. Observe that $\hat{a}_{i}$ is a multiple of $2^{m-2 i}$. Indeed,

$$
\hat{P}_{m}(z)= \begin{cases}\sum_{j=0}^{\kappa} \pi_{j} 2 \tilde{C}_{m-2 j}(z), & \text { if } m \text { is odd } \\ \pi_{\kappa}+\sum_{j=0}^{\kappa-1} \pi_{j} 2 \tilde{C}_{m-2 j}(z), & \text { if } m \text { is even }\end{cases}
$$

where $\kappa=[m / 2]$. But the polynomial $2 \tilde{C}_{k}(z / 2)$ has integer coefficients, for every $k \in \mathbb{N}$. By (3.31), the same holds for the polynomial $\hat{P}_{m}(z / 2)$, whence our claim follows. Hence $\hat{a}_{i}=\hat{b}_{i} 2^{m-2 i}$ where $\hat{b}_{i}$ is an integer. In particular, $\hat{a}_{0}=2^{m}$, that is, $\hat{b}_{0}=1$. Finally, since $\hat{a}_{\mu} \neq 0$, we have also $\hat{b}_{\mu} \neq 0$.

Let us now recall the following classical identity:

$$
(2 z)^{n} \tilde{C}_{k}(z)=\sum_{\nu=0}^{n} C_{n}^{\nu} \tilde{C}_{k+n-2 \nu}(z), n \in \mathbb{N}, k \in \mathbb{Z},
$$

which may be easily proved by induction, the case $n=1$ representing just the well-known recurrence relation between Chebyshev polynomials of first kind:

$$
2 z \tilde{C}_{k}(z)=\tilde{C}_{k-1}(z)+\tilde{C}_{k+1}(z), k \in \mathbb{Z} .
$$


Applying (3.32) with $k=0$, we obtain:

$$
\begin{aligned}
\hat{P}_{m}(z) & =\sum_{i=0}^{\mu} \hat{a}_{i} z^{m-2 i}=\sum_{i=0}^{\mu} \hat{b}_{i}(2 z)^{m-2 i} \\
& =\sum_{i=0}^{\mu} \hat{b}_{i} \sum_{k=0}^{m-2 i} C_{m-2 i}^{k} \tilde{C}_{m-2 i-2 k}(z)=\sum_{j=0}^{m}\left(\sum_{i=0}^{\mu} \hat{b}_{i} C_{m-2 j}^{j-i}\right) \tilde{C}_{m-2 j}(z),
\end{aligned}
$$

whence (3.22) follows by a simple comparison of the coefficients.

b) and c) Transforming (3.21) gives:

$$
\begin{aligned}
\tilde{P}_{m}(r) \mathbb{E}\left(r^{-V}\right)=1 & \Leftrightarrow \hat{P}_{m}(r) \mathbb{E}\left(r^{-V}\right)=\sum_{j=0}^{m} \pi_{j} \\
& \Leftrightarrow \sum_{i=0}^{\mu} \hat{b}_{i}(2 r)^{m-2 i} \sum_{j=0}^{+\infty} q_{j} r^{-m-2 j}=\sum_{j=0}^{m} \pi_{j} \\
& \Leftrightarrow \sum_{i=0}^{\mu} \hat{b}_{i}(2 r)^{m-2 i} \sum_{j=0}^{+\infty} \hat{q}_{j}(2 r)^{-m-2 j}=1,
\end{aligned}
$$

where the $\left(\hat{q}_{j}, j \geq 0\right)$ are defined by (3.23). By substituting $(2 r)^{-1}=z$ in the last equality and simplifying, we are finally led to

$$
\sum_{i=0}^{\mu} \hat{b}_{i} z^{i} \sum_{j=0}^{+\infty} \hat{q}_{j} z^{j}=1,
$$

whence the system (3.24) follows at once. By induction it is easily seen that $\hat{q}_{k}$ is always an integer.

d) Compute the coefficients $\hat{q}_{0}, \hat{q}_{1}, \ldots, \hat{q}_{\mu-1}$ in terms of $\hat{b}_{0}, \hat{b}_{1}, \ldots, \hat{b}_{\mu-1}$, by using equations $\left(E_{0}\right),\left(E_{1}\right), \ldots,\left(E_{\mu-1}\right)$. Consider the following square matrix with $\nu:=\mu-1$ rows and columns:

$$
W=\left(\begin{array}{ccccccccc}
1 & 0 & \ldots & 0 & \ldots & 1 & 0 & \ldots & 0 \\
z_{1} & z_{1} & \ldots & z_{1} & \ldots & z_{w} & z_{w} & \ldots & z_{w} \\
z_{1}^{2} & 2 z_{1}^{2} & \ldots & 2^{m_{1}-1} z_{1}^{2} & \ldots & z_{w}^{2} & 2 z_{w}^{2} & \ldots & 2^{m_{w}-1} z_{w}^{2} \\
\ldots & \ldots & \ldots & \ldots & \ldots & \ldots & \ldots & \ldots & \ldots \\
z_{1}^{k} & k z_{1}^{k} & \ldots & k^{m_{1}-1} z_{1}^{k} & \ldots & z_{w}^{k} & k z_{w}^{k} & \ldots & k^{m_{w}-1} z_{w}^{k} \\
\ldots & \ldots & \ldots & \ldots & \ldots & \ldots & \ldots & \ldots & \ldots \\
z_{1}^{\nu} & \nu z_{1}^{\nu} & \ldots & \nu^{m_{1}-1} z_{1}^{\nu} & \ldots & z_{w}^{\nu} & \nu z_{w}^{\nu} & \ldots & \nu^{m_{\nu}-1} z_{w}^{\nu}
\end{array}\right)
$$

Classically, $\operatorname{det} W \neq 0$, since the roots $z_{i}$ of the generating polynomial $\hat{B}$ are non-zero and distinct. Thus, the system $Y=W C$, where $Y=\left(\hat{q}_{0}, \ldots, \hat{q}_{\mu-1}\right)^{t}$ and $C=\left(c_{1,0}, \ldots, c_{1, m_{1}-1}, \ldots, c_{w, 0}, \ldots c_{w, m_{w}-1}\right)^{t}$, has a unique solution $C$. 
In other words, the representation formula (3.26) holds for $k<\mu$. So it remains to show that it is still valid for $k \geq \mu$, with the constants $c_{s, r}$ now adjusted as above, or equivalently, that $\hat{q}_{k}$ satisfies equation $\left(\mathrm{E}_{k}\right)$, for every $k \geq \mu$. By linearity, it is enough to verify the equation separately for each term of the form $y_{k}=k^{r} z_{s}^{k}, 1 \leq s \leq w, 0 \leq r<m_{s}$ :

$$
\sum_{i=0}^{\mu} \hat{b}_{i} y_{k-i}=\sum_{i=0}^{\mu} \hat{b}_{i}(k-i)^{r} z_{s}^{(k-i)}=z_{s}^{k-\mu} \sum_{i=0}^{\mu} \hat{b}_{i}(k-i)^{r} z_{s}^{\mu-i}=0,
$$

since $(k-i)^{r}$ is a polynomial of degree $<m_{s}$.

Finally, because the roots of $\hat{P}_{m}$ are all located in the open unit disk and

$$
\hat{B}\left(4 z^{2}\right)=\sum_{i=0}^{\mu} \hat{b}_{i}(2 z)^{2(\mu-i)}=(2 z)^{-m_{0}} \sum_{i=0}^{\mu} \hat{a}_{i} z^{m-2 i}=(2 z)^{-m_{0}} \hat{P}_{m}(z),
$$

it is clear that $\left|z_{i}\right|<4$, for every $1 \leq i \leq w$.

e) By (3.33), we have

$$
\hat{P}_{m}(r) \sum_{i=0}^{+\infty} \hat{q}_{i}(2 r)^{-2 i}=(2 r)^{m}
$$

Thus,

$$
\begin{aligned}
Q_{k}(r) & =(2 r)^{m+2 k} \frac{\sum_{i>k} q_{i} r^{-2 i}}{\sum_{i=0}^{+\infty} q_{i} r^{-2 i}} \\
& =(2 r)^{m+2 k} \frac{\sum_{i>k} \hat{q}_{i}(2 r)^{-2 i}}{\sum_{i=0}^{+\infty} \hat{q}_{i}(2 r)^{-2 i}}=(2 r)^{2 k} \hat{P}_{m}(r) \sum_{i>k} \hat{q}_{i}(2 r)^{-2 i} .
\end{aligned}
$$

From (3.35), (3.32) and the fact that $\hat{q}_{0}=1$, we derive that

$$
\begin{aligned}
Q_{0}(r) & =\hat{P}_{m}(r) \sum_{i>0} \hat{q}_{i}(2 r)^{-2 i}=\hat{P}_{m}(r) \sum_{i \geq 0} \hat{q}_{i}(2 r)^{-2 i}-\hat{q}_{0} \hat{P}_{m}(r) \\
& =(2 r)^{m}-\hat{P}_{m}(r)=\sum_{j=1}^{m-1}\left(C_{m}^{j}-\pi_{j}\right) \tilde{C}_{m-2 j}(r),
\end{aligned}
$$

which implies in particular that $\hat{\theta}_{j, 0}=C_{m}^{j}-\pi_{j}$. More generally, the following recurrence relation holds between $Q_{k}$ and $Q_{k-1}$ :

$Q_{k}(r)=(2 r)^{2 k} \hat{P}_{m}(r)\left(\sum_{i>k-1} \hat{q}_{i}(2 r)^{-2 i}-\hat{q}_{k}(2 r)^{-2 k}\right)=(2 r)^{2} Q_{k-1}(r)-\hat{q}_{k} \hat{P}_{m}(r)$.

Iterating this, we get

$$
Q_{k}(r)=(2 r)^{m+2 k}-\hat{P}_{m}(r) \sum_{l=0}^{k} \hat{q}_{k-l}(2 r)^{2 l} .
$$


Expanding the last product yields

$$
\begin{aligned}
Q_{k}(r) & =(2 r)^{m+2 k}-\sum_{i=0}^{\mu} \sum_{l=0}^{k} \hat{b}_{i} \hat{q}_{k-l}(2 r)^{m-2(i-l)} \\
& =(2 r)^{m+2 k}-\sum_{h=-k}^{\mu}(2 r)^{m-2 h} \sum_{i=0 \vee h}^{\mu \wedge(k+h)} \hat{b}_{i} \hat{q}_{k+h-i} .
\end{aligned}
$$

The term corresponding to $h=-k$ is exactly $(2 r)^{m+2 k}$. The other terms with index $h \leq 0$ vanish according to equation $\left(E_{k}\right)$. Hence,

$$
Q_{k}(r)=-\sum_{h=1}^{\mu}(2 r)^{m-2 h} \sum_{i=h}^{\mu \wedge(k+h)} \hat{b}_{i} \hat{q}_{k+h-i}=\sum_{h=1}^{\mu}(2 r)^{m-2 h} \sum_{i=0}^{h-1} \hat{b}_{i} \hat{q}_{k+h-i} .
$$

We see already here that $Q_{k}$ has integer coefficients and degree at most $m-2$. Using again (3.32), we now express $Q_{k}$ in terms of the Chebyshev polynomials:

$$
\begin{aligned}
Q_{k}(r) & =\sum_{h=1}^{\mu} \sum_{i=0}^{h-1} \hat{b}_{i} \hat{q}_{k+h-i} \sum_{\nu=0}^{m-2 h} C_{m-2 h}^{\nu} \tilde{C}_{m-2 h-2 \nu}(r) \\
& =\sum_{j=1}^{m-1} \tilde{C}_{m-2 j}(r) \sum_{h=1}^{\mu} C_{m-2 h}^{j-h} \sum_{i=0}^{h-1} \hat{b}_{i} \hat{q}_{k+h-i},
\end{aligned}
$$

whence (3.27) follows by setting

$$
\hat{\theta}_{j, k}=\sum_{h=1}^{\mu} C_{m-2 h}^{j-h} \sum_{i=0}^{h-1} \hat{b}_{i} \hat{q}_{k+h-i} .
$$

The other claims are obvious.

f) Substituting $r=\operatorname{ch} \lambda$ in (3.27), we get

$$
Q_{k}(\operatorname{ch} \lambda)=\sum_{j=1}^{m-1} \hat{\theta}_{j, k} \operatorname{ch}(\lambda(m-2 j))=\sum_{j=1}^{m-1} \hat{\theta}_{j, k} \exp (\lambda(m-2 j)),
$$

where the last equality comes from the symmetry of the coefficients. The proof of (3.29) is achieved by substituting $u=e^{-\lambda}$ in (3.37).

g) The recurrence relation is easily obtained by writing both members of the equality

$$
Q_{k}(r)=(2 r)^{2} Q_{k-1}(r)-\hat{q}_{k} \hat{P}_{m}(r)
$$

in terms of the Chebyshev polynomials. 
We end this paragraph with an equivalent formulation of Proposition 3.1.

Theorem 3.1 (Characterization of stopping distributions) With the notation of Proposition 3.2, a distribution

$$
\mathcal{L}=\left\{\left(m-2 j, p_{j}\right), 0 \leq j \leq m\right\}
$$

satisfying conditions $\left(N_{1}\right)$ to $\left(N_{3}\right)$ is a stopping distribution if and only if the following hold:

i) the coefficients in the power series expansion of $1 / \tilde{P}_{m}(1 / r)$ are all $\geq 0$,

ii) the coefficients of the polynomials $\left(\hat{Q}_{k}\right)_{k \geq 0}$ are all $\geq 0$.

Remark 3.3 Condition ii) cannot be omitted in this theorem, since we have the following counter-example for $m=7$ :

$\mathcal{L}=\left\{\left(7, \frac{1}{16}\right),(5,0),\left(3, \frac{7}{16}\right),(1,0),(-1,0),\left(-3, \frac{7}{16}\right),(-5,0),\left(-7, \frac{1}{16}\right)\right\}$.

Here, i) holds but ii) does not, since some negative coefficients appear in $\hat{Q}_{2}$. As a result, $\mathcal{L}$ is not a stopping distribution.

Notation In view of Remark 3.2, (i), any probability distribution

$$
\mathcal{L}=\left\{\left(m-2 j, p_{j}\right), 0 \leq j \leq m\right\}
$$

satisfying conditions $\left(N_{1}\right)$ to $\left(N_{3}\right)$, will be denoted throughout by

$$
\mathcal{L}_{m}\left(\hat{b}_{0}, \hat{b}_{1}, \ldots, \hat{b}_{\mu}\right)
$$

where the integers $\mu$ and $\hat{b}_{0}, \hat{b}_{1}, \ldots, \hat{b}_{\mu}$ are defined in Proposition 3.2.

Remark 3.4 Given $T_{0} \in \mathcal{T}$ such that

$$
\mathcal{L}\left(S_{T_{0}}\right)=\mathcal{L}_{m}\left(\hat{b}_{0}, \hat{b}_{1}, \ldots, \hat{b}_{\mu}\right)
$$

we may consider, for $n \geq 0, T_{n}:=T_{0}+n$. Then, $T_{n} \in \mathcal{T}$ and it is easily seen that:

$$
\mathcal{L}\left(S_{T_{n}}\right)=\mathcal{L}_{m+n}\left(\hat{b}_{0}, \hat{b}_{1}, \ldots, \hat{b}_{\mu}\right) .
$$

Moreover, $S_{T_{n}}$ has the same generating polynomial (in the sense of Proposition 3.2) than $S_{T_{0}}$, for every $n \geq 0$. 


\subsection{A version of Example 4 in the natural filtration}

In the case where $m$ is even, we are able to construct a "natural" version of the stopping time $\hat{T}$ given by (2.28) (cf. also Remark 2.7 (ii)). This will be helpful to establish the complete list of stopping distributions for $m \leq 4$.

Proposition 3.3 Let $T \in \mathcal{T}$ and $\mathcal{L}=\mathcal{L}\left(S_{T}\right)=\left\{\left(m-2 j, p_{j}\right), 0 \leq j \leq m\right\}$, where $m=\min T$ is assumed to be even. In particular, conditions $\left(N_{1}\right)$ to $\left(N_{3}\right)$ are satisfied. Let $\left(\hat{\pi}_{j}, 0 \leq j \leq m\right)$ be a sequence of weights differing from the $\left(\pi_{j}, 0 \leq j \leq m\right)$ only by the central coefficient, i.e.

$$
0 \leq \hat{\pi}_{\frac{m}{2}} \leq \pi_{\frac{m}{2}} \text { and } j \neq \frac{m}{2} \Rightarrow \hat{\pi}_{j}=\pi_{j},
$$

and define

$$
\hat{p}_{j}=\frac{\hat{\pi}_{j}}{\sum_{l=0}^{m} \hat{\pi}_{l}} .
$$

Then $\hat{\mathcal{L}}=\left\{\left(m-2 j, \hat{p}_{j}\right) / 0 \leq j \leq m\right\}$, is also a stopping distribution. It can be realized via the stopping time $\hat{T}$ given by (3.43).

Proof. Consider the increasing sequence of stopping times $\left(T_{n}, n \geq 0\right)$ introduced in Example 4, defined by (2.27). For every $k \geq 0$, choose a set of paths $\Gamma_{k} \subset\left\{T=m+2 k, S_{T}=0\right\}$ such that

$$
\left|\Gamma_{k}\right|=\pi_{0, k} \hat{\pi} \frac{m}{2}, k \geq 0 .
$$

This is clearly possible because

$$
\pi_{0, k} \hat{\pi}_{\frac{m}{2}} \leq \pi_{0, k} \pi_{\frac{m}{2}}=\pi_{\frac{m}{2}, k}
$$

Note that if $\pi_{0, k}=0$, i.e. if $\mathbb{P}(T=m+2 k)=0$ then $\Gamma_{k}=\emptyset$. We have:

$$
\mathbb{P}\left(\Gamma_{k}\right)=\pi_{0, k} \cdot \hat{\pi}_{\frac{m}{2}} \cdot 2^{-m-2 k} \text { and } \Gamma:=\bigcup_{k=0}^{\infty} \Gamma_{k} \in \mathcal{F}_{T}
$$

Now, for every $n \geq 1$, we define:

$$
Y_{n}= \begin{cases}\mathbb{I}_{\Gamma} \circ \theta_{T_{n-1}} & \text { if } S_{T_{n-1}}=0 \\ 0 & \text { otherwise }\end{cases}
$$

$Y_{n}$ is obviously $\mathcal{F}_{T_{n}}$-measurable. (3.39) ensures that $Y_{n} \Perp T_{n}-T_{n-1}$, conditionally on $\left\{S_{T_{n-1}}=0\right\}$. To see this, it is enough, by the Markov property, to consider the case $n=1$ :

$$
\mathbb{P}\left(Y_{1}=1 \text { and } T_{1}=m+2 k\right)=\mathbb{P}\left(\Gamma_{k}\right)=\pi_{0, k} \cdot \hat{\pi}_{\frac{m}{2}} \cdot 2^{-m-2 k} .
$$


Summing over $k$ and using (3.4) and (3.9), we get

$$
\begin{aligned}
\mathbb{P}\left(Y_{1}=1\right) & =\sum_{k=0}^{+\infty} \pi_{0, k} \cdot \hat{\pi}_{\frac{m}{2}} \cdot 2^{-m-2 k} \\
& =\frac{\hat{\pi} \frac{m}{2}}{\pi \frac{m}{2}} \sum_{k=0}^{+\infty} \pi_{0, k} \cdot \pi \frac{m}{2} \cdot 2^{-m-2 k}=\frac{\hat{\pi} \frac{m}{2}}{\pi \frac{m}{2}} \mathbb{P}\left(S_{T}=0\right) .
\end{aligned}
$$

Since $T_{1}=T$ a.s. and $T \Perp S_{T}$, we obtain similarly:

$$
\mathbb{P}\left(T_{1}=m+2 k\right)=\mathbb{P}\left(T_{1}=m+2 k / S_{T}=0\right)=\frac{\pi_{0, k} \cdot \pi \frac{m}{2} \cdot 2^{-m-2 k}}{\mathbb{P}\left(S_{T}=0\right)},
$$

whence the independence of $Y_{1}$ and $T_{1}$ comes immediately.

Now, our key observation is that the law of $Y_{1}$, conditionally on $\left\{S_{T_{1}}=0\right\}$ and $\left\{T_{1}=m+2 k\right\}$, does not depend on $k$ :

$$
\mathbb{P}\left(Y_{1}=1 / S_{T_{1}}=0 \text { and } T_{1}=m+2 k\right)=\frac{\pi_{0, k} \cdot \hat{\pi}_{\frac{m}{2}} \cdot 2^{-m-2 k}}{\pi_{0, k} \cdot \pi_{\frac{m}{2}} \cdot 2^{-m-2 k}}=\frac{\hat{\pi} \frac{m}{2}}{\pi \frac{m}{2}}=\rho
$$

and likewise,

$$
\mathbb{P}\left(Y_{1}=0 / S_{T_{1}}=0 \text { and } T_{1}=m+2 k\right)=1-\frac{\hat{\pi} \frac{m}{2}}{\pi \frac{m}{2}}=\sigma
$$

To define the stopping time $\hat{T}$ solving our problem, it suffices to copy (2.28):

$$
\hat{T}=T_{n} \Leftrightarrow\left\{\begin{array} { c } 
{ S _ { T _ { 1 } } = 0 } \\
{ Y _ { 1 } = 0 }
\end{array} \text { and } \ldots \text { and } \left\{\begin{array} { c } 
{ S _ { T _ { n - 1 } } = 0 } \\
{ Y _ { n - 1 } = 0 }
\end{array} \text { and } \left\{\begin{array}{c}
S_{T_{n}} \neq 0 \\
\text { or } Y_{n}=1 .
\end{array}\right.\right.\right.
$$

Clearly, $\hat{T}$ satisfies the identity in law (2.31). Therefore, the rest of the proof goes exactly as in Proposition 2.6: (2.33) still holds by (3.41) and (3.42). Finally, the distribution of $S_{\hat{T}}$ is easily derived by computing explicitly the Laplace transform $\psi_{\hat{T}}$, given by (2.29).

Notation The above stopping time $\hat{T}$ depends on the associated stopping time $T$ and, of course, on the choice of the set $\Gamma$. Hence, there are infinitely many such stopping times. We denote them all by $\hat{T}\left(\hat{\pi}_{m / 2}\right)$, where $\hat{\pi}_{m / 2}=\left|\Gamma_{0}\right|$, without bothering about the choice of $\Gamma$. This is justified by the fact that we are only interested in the distributions of $\hat{T}$ and $S_{\hat{T}}$. 


\subsection{Stopping distributions for $m \in\{1,2,3,4\}$}

We assume that the notations for the various stopping times introduced in the previous paragraphs are still valid.

$m=1$ : there is exactly 1 stopping distribution:

$$
\left\{\left(1, \frac{1}{2}\right),\left(-1, \frac{1}{2}\right)\right\} \quad \text { realized via } T=1
$$

$m=2$ : there are 3 stopping distributions:

$$
\begin{array}{lll}
\left\{\left(2, \frac{1}{2}\right),\left(-2, \frac{1}{2}\right)\right\} & \text { realized via } & T_{2}^{*} \\
\left\{\left(2, \frac{1}{3}\right),\left(0, \frac{1}{3}\right),\left(-2, \frac{1}{3}\right)\right\} & & V_{2} \\
\left\{\left(2, \frac{1}{4}\right),\left(0, \frac{2}{4}\right),\left(-2, \frac{1}{4}\right)\right\} & & T=2
\end{array}
$$

$m=3$ : there are 4 stopping distributions:

$$
\begin{array}{lll}
\left\{\left(3, \frac{1}{2}\right),\left(-3, \frac{1}{2}\right)\right\} & \text { realized via } & T_{3}^{*} \\
\left\{\left(3, \frac{1}{4}\right),\left(1, \frac{1}{4}\right),\left(-1, \frac{1}{4}\right),\left(-3, \frac{1}{4}\right)\right\} & T_{2}^{*}+1, V_{3}, \text { or } \Lambda_{3} \\
\left\{\left(3, \frac{1}{6}\right),\left(1, \frac{2}{6}\right),\left(-1, \frac{2}{6}\right),\left(-3, \frac{1}{6}\right)\right\} & V_{2}+1 \\
\left\{\left(3, \frac{1}{8}\right),\left(1, \frac{3}{8}\right),\left(-1, \frac{3}{8}\right),\left(-3, \frac{1}{8}\right)\right\} & T=3
\end{array}
$$

$m=4:$ there are 22 stopping distributions:

$$
\begin{array}{lll}
\left\{\left(4, \frac{1}{2}\right),\left(-4, \frac{1}{2}\right)\right\} & \text { realized via } & T_{4}^{*} \\
\left\{\left(4, \frac{1}{3}\right),\left(0, \frac{1}{3}\right),\left(-4, \frac{1}{3}\right)\right\} & & \hat{T}_{2,2}^{*}(1) \\
\left\{\left(4, \frac{1}{4}\right),\left(0, \frac{2}{4}\right),\left(-4, \frac{1}{4}\right)\right\} & & T_{2,2}^{*}
\end{array}
$$

(When $\pi_{0}=1, \pi_{1}=0$ and $\pi_{2} \geq 3$, the power series $1 / \tilde{P}_{m}(1 / r)$ contains negative coefficients.)

$$
\begin{array}{ll}
\left\{\left(4, \frac{1}{4}\right),\left(2, \frac{1}{4}\right),\left(-2, \frac{1}{4}\right),\left(-4, \frac{1}{4}\right)\right\} & T_{3}^{*}+1 \\
\left\{\left(4, \frac{1}{5}\right),\left(2, \frac{1}{5}\right),\left(0, \frac{1}{5}\right)\left(-2, \frac{1}{5}\right),\left(-4, \frac{1}{5}\right)\right\} & V_{4} \\
\left\{\left(4, \frac{1}{6}\right),\left(2, \frac{1}{6}\right),\left(0, \frac{2}{6}\right)\left(-2, \frac{1}{6}\right),\left(-4, \frac{1}{6}\right)\right\} & T_{2}^{*}+V_{2} \circ \theta_{T_{2}^{*}}
\end{array}
$$

(When $\pi_{0}=1, \pi_{1}=1$ and $\pi_{2} \geq 3$, the power series $1 / \tilde{P}_{m}(1 / r)$ contains negative coefficients.)

$$
\begin{array}{ll}
\left\{\left(4, \frac{1}{6}\right),\left(2, \frac{2}{6}\right),\left(-2, \frac{2}{6}\right),\left(-4, \frac{1}{6}\right)\right\} & \Lambda_{4} \\
\left\{\left(4, \frac{1}{7}\right),\left(2, \frac{2}{7}\right),\left(0, \frac{1}{7}\right),\left(-2, \frac{2}{7}\right),\left(-4, \frac{1}{7}\right)\right\} & \hat{U}(1) \text { (for } U, \text { cf. infra) } \\
\left\{\left(4, \frac{1}{8}\right),\left(2, \frac{2}{8}\right),\left(0, \frac{2}{8}\right),\left(-2, \frac{2}{8}\right),\left(-4, \frac{1}{8}\right)\right\} & U=T_{2}^{*}+2 \\
\left\{\left(4, \frac{1}{9}\right),\left(2, \frac{2}{9}\right),\left(0, \frac{3}{9}\right),\left(-2, \frac{2}{9}\right),\left(-4, \frac{1}{9}\right)\right\} & V_{2}+V_{2} \circ \theta_{V_{2}}
\end{array}
$$

(When $\pi_{0}=1, \pi_{1}=2$ and $\pi_{2} \geq 4$, the power series $1 / \tilde{P}_{m}(1 / r)$ contains negative coefficients.) 


$$
\begin{array}{ll}
\left\{\left(4, \frac{1}{8}\right),\left(2, \frac{3}{8}\right),\left(-2, \frac{3}{8}\right),\left(-4, \frac{1}{8}\right)\right\} & \hat{Y}(0) \text { (for } Y, \text { cf. infra) } \\
\left\{\left(4, \frac{1}{9}\right),\left(2, \frac{3}{9}\right),\left(0, \frac{1}{9}\right),\left(-2, \frac{3}{9}\right),\left(-4, \frac{1}{9}\right)\right\} & \hat{Y}(1) \\
\left\{\left(4, \frac{1}{10}\right),\left(2, \frac{3}{10}\right),\left(0, \frac{2}{10}\right),\left(-2, \frac{3}{10}\right),\left(-4, \frac{1}{10}\right)\right\} & \hat{Y}(2) \\
\left\{\left(4, \frac{1}{11}\right),\left(2, \frac{3}{11}\right),\left(0, \frac{3}{11}\right),\left(-2, \frac{3}{11}\right),\left(-4, \frac{1}{11}\right)\right\} & \hat{Y}(3) \\
\left\{\left(4, \frac{1}{12}\right),\left(2, \frac{3}{12}\right),\left(0, \frac{4}{12}\right),\left(-2, \frac{3}{12}\right),\left(-4, \frac{1}{12}\right)\right\} & Y=V_{2}+2
\end{array}
$$

(When $\pi_{0}=1, \pi_{1}=3$ and $\pi_{2} \geq 5$, the power series $1 / \tilde{P}_{m}(1 / r)$ contains negative coefficients.)

$$
\begin{array}{lll}
\left\{\left(4, \frac{1}{10}\right),\left(2, \frac{4}{10}\right),\left(-2, \frac{4}{10}\right),\left(-4, \frac{1}{10}\right)\right\} & \hat{C}(0) \text { (for } C, \text { cf. infra) } \\
\left\{\left(4, \frac{1}{11}\right),\left(2, \frac{4}{11}\right),\left(0, \frac{1}{11}\right),\left(-2, \frac{4}{11}\right),\left(-4, \frac{1}{11}\right)\right\} & \hat{C}(1) \\
\left\{\left(4, \frac{1}{12}\right),\left(2, \frac{4}{12}\right),\left(0, \frac{2}{12}\right),\left(-2, \frac{4}{12}\right),\left(-4, \frac{1}{12}\right)\right\} & \hat{C}(2) \\
\left\{\left(4, \frac{1}{13}\right),\left(2, \frac{4}{13}\right),\left(0, \frac{3}{13}\right),\left(-2, \frac{4}{13}\right),\left(-4, \frac{1}{13}\right)\right\} & \hat{C}(3) \\
\left\{\left(4, \frac{1}{14}\right),\left(2, \frac{4}{14}\right),\left(0, \frac{4}{14}\right),\left(-2, \frac{4}{14}\right),\left(-4, \frac{1}{14}\right)\right\} & \hat{C}(4) \\
\left\{\left(4, \frac{1}{15}\right),\left(2, \frac{4}{15}\right),\left(0, \frac{5}{15}\right),\left(-2, \frac{4}{15}\right),\left(-4, \frac{1}{15}\right)\right\} & \hat{C}(5) \\
\left\{\left(4, \frac{1}{16}\right),\left(2, \frac{4}{16}\right),\left(0, \frac{6}{16}\right),\left(-2, \frac{4}{16}\right),\left(-4, \frac{1}{16}\right)\right\} & C=4
\end{array}
$$

\subsection{Generating polynomials of degree $\leq 2$. Stopping distributions for $m=5$}

By Proposition 3.2, when $m \leq 5$, the generating polynomial $\hat{B}$ of a stopping distribution, given by (3.25), is of degree $\mu \leq 2$. We are now going to establish the complete list of these generating polynomials and derive therefrom the stopping distributions in the case $m=5$.

If $\mu=0$, then $\hat{B}(z)=1$ and $T$ is constant. $\mathcal{L}_{m}(1)$ is nothing else but $\mathcal{B S}(m)$ and of course, this is a stopping distribution for every $m \geq 1$.

If $\mu=1$, then $\hat{B}(z)=z+\hat{b}_{1}$ has exactly one root, $\alpha=-\hat{b}_{1}$, which is non-zero.

Proposition 3.4 If $\mathcal{L}_{m}(1,-\alpha)$, where $m>0$, is a stopping distribution, then necessarily $\alpha=1,2$ or 3 . Conversely, $\mathcal{L}_{m}(1,-1)$ and $\mathcal{L}_{m}(1,-2)$ are stopping distributions, whenever $m \geq 2$, and so is $\mathcal{L}_{m}(1,-3)$, whenever $m \geq 3$.

Proof. By (3.26), $\hat{q}_{k}=\alpha^{k}$ and by (3.23), $q_{k}=(1-\alpha / 4)(\alpha / 4)^{k}$, whence $\alpha=1,2$ or 3 . For the converse, given a distribution of this type, it is easy to see that

$$
Q_{k}(r)=(2 r)^{m+2 k} \frac{\sum_{i>k}\left(\frac{\alpha}{4 r^{2}}\right)^{i}}{\sum_{i=0}^{+\infty}\left(\frac{\alpha}{4 r^{2}}\right)^{i}}=\alpha^{k+1}(2 r)^{m-2},
$$


so that,

$$
\hat{Q}_{k}(r)=r^{m} Q_{k}\left(\frac{r^{2}+1}{2 r}\right)=\alpha^{k+1} r^{2}\left(r^{2}+1\right)^{m-2}=\alpha^{k+1} \sum_{j=1}^{m-1} C_{m-2}^{j-1} r^{2 j},
$$

which has only nonnegative coefficients, for every $\alpha>0$. Now, according to $(3.22)$, the corresponding $\pi_{j}$ are given by

$$
\pi_{j}=C_{m}^{j}-\alpha C_{m-2}^{j-1}, 0 \leq j \leq m .
$$

A simple calculation shows that $0 \leq \pi_{j} \leq C_{m}^{j}$, for every $0 \leq j \leq m$, provided that

$$
\begin{cases}\frac{\alpha}{4} \leq \frac{m-1}{m} & \text { when } m \text { is even, } \\ \frac{\alpha}{4} \leq \frac{m}{m+1} & \text { when } m \text { is odd, }\end{cases}
$$

whence the proposition follows immediately.

Let us finally investigate the case $\mu=2$. Given two integers $\hat{b}_{1}$ and $\hat{b}_{2}$, the generating polynomial of $\mathcal{L}_{m}\left(\hat{b}_{1}, \hat{b}_{2}\right)$ has two non-zero roots $\alpha$ and $\beta$, not necessarily distinct and satisfying

$$
\alpha+\beta=-\hat{b}_{1} \quad \text { and } \quad \alpha \beta=\hat{b}_{2} .
$$

$\alpha$ and $\beta$ are either both real or conjugate complex and distinct. The following lemma shows that, in the second case, $\mathcal{L}_{m}\left(\hat{b}_{1}, \hat{b}_{2}\right)$ cannot be a stopping distribution, for any $m$.

Lemma 3.1 Let $\alpha$ and $\beta$ be defined by (3.44). Then:

a) The coefficients $\hat{q}_{k}$ in (3.24) are given by

$$
\hat{q}_{k}=\left\{\begin{array}{ll}
\frac{\alpha^{k+1}-\beta^{k+1}}{\alpha-\beta} & \text { if } \alpha \neq \beta \\
(k+1) \alpha^{k+1} & \text { if } \alpha=\beta
\end{array}, k \geq 0 .\right.
$$

b) If $\hat{q}_{k} \geq 0$, for every $k \geq 0$, then $\alpha$ and $\beta$ are both real.

Proof. Statement a) follows immediately from the recurrence relation

$$
\hat{q}_{k}=(\alpha+\beta) \hat{q}_{k-1}-\alpha \beta \hat{q}_{k-2}, k \geq 2,
$$

with initial conditions $\hat{q}_{0}=1$ and $\hat{q}_{1}=\alpha+\beta$.

b) Assuming that $\alpha=r e^{i \theta}$ and $\beta=r e^{-i \theta}$, for some $\theta \neq 0$, we have

$$
\hat{q}_{k}=\frac{\alpha^{k+1}-\bar{\alpha}^{k+1}}{\alpha-\bar{\alpha}}=r^{k} \frac{\sin ((k+1) \theta)}{\sin (\theta)}, k \geq 0,
$$

whence the claim can be derived without difficulty. 
The analog of Proposition 3.4 in the case $\mu=2$ is

Proposition 3.5 For any integers $s \geq 0$ and $p \neq 0$, the following are equivalent:

(i) $\mathcal{L}_{m}(1,-s, p)$ is a stopping distribution, for sufficiently large $m$,

(ii) The roots $\alpha$ and $\beta$ of $z^{2}-s z+p=0$ are real and $|\alpha|<4,|\beta|<4$.

Proof. (i) $\Rightarrow$ (ii): We know already by Lemma 3.1 that $\alpha$ and $\beta$ must be real. Moreover, since $\hat{q}_{1}=\alpha+\beta$, necessarily $s \geq 0$. By (3.23) and (3.45), we have

$$
q_{k}=\frac{(4-\alpha)(4-\beta)}{4^{k+2}} \cdot \begin{cases}\frac{\alpha^{k+1}-\beta^{k+1}}{\alpha-\beta} & \text { if } \alpha \neq \beta \\ (k+1) \alpha^{k+1} & \text { if } \alpha=\beta\end{cases}
$$

From this and the fact that $\lim _{k \rightarrow+\infty} q_{k}=0$, it follows that $|\alpha|<4$ and $|\beta|<4$.

(ii) $\Rightarrow$ (i): Assume for instance that $\alpha \geq \beta$. Then, since $s \geq 0$ and $p \neq 0$, necessarily $\alpha \geq|\beta|>0$, and therefore the $\hat{q}_{k}$, given by (3.45) are all $\geq 0$. The same holds for the $q_{k}$, since $|\alpha|<4$ and $|\beta|<4$.

Now, by (3.28) and (3.46),

$$
\hat{\theta}_{j, k}=C_{m-2}^{j-1} \hat{q}_{k+1}+C_{m-4}^{j-2}\left(\hat{q}_{k+2}-s \hat{q}_{k+1}\right)=C_{m-2}^{j-1} \hat{q}_{k+1}-C_{m-4}^{j-2} p \hat{q}_{k} .
$$

We have to show that the $\hat{\theta}_{j, k}$ are all $\geq 0$, provided that $m$ is large enough. If $p<0$, this is obviously true. If $p>0$, we have $s>\alpha>\beta>0$ and

$$
\forall j \in\{0,1, \ldots, m\}, \forall k \geq 0, \hat{\theta}_{j, k} \geq 0 \Leftrightarrow \sup _{k \geq 0} \frac{p \hat{q}_{k}}{\hat{q}_{k+1}} \leq \min _{0 \leq j \leq m} \frac{C_{m-2}^{j-1}}{C_{m-4}^{j-2}} .
$$

The right-hand side of the last inequality is

$$
\min _{0 \leq j \leq m} \frac{C_{m-2}^{j-1}}{C_{m-4}^{j-2}}= \begin{cases}\frac{4(m-3)}{m-2} & \text { if } m \text { is even, } \\ \frac{4(m-3)}{m-1} & \text { if } m \text { is odd. }\end{cases}
$$

To simplify the left-hand side, let us define $u_{k}=\hat{q}_{k+1} / \hat{q}_{k}, k \geq 0$. We have $u_{0}=s \neq 0$ and, for $k \geq 1$, the following recurrence holds:

$$
u_{k}=s-\frac{p}{u_{k-1}} \text {. }
$$

A simple fixed-point argument shows that $\left(u_{k}, k \geq 0\right)$ decreases to $\alpha$. Hence, $\inf \left\{u_{k}, k \geq 0\right\}=\alpha$, so that

$$
\sup _{k \geq 0} \frac{p \hat{q}_{k}}{\hat{q}_{k+1}}=\frac{p}{\alpha}=\beta<4 .
$$

Thus, for $m$ large enough, (3.48) is satisfied. 
To complete the proof, it remains to check that the $\pi_{j}$ are all nonnegative, for sufficiently large $m$. We have

$$
\pi_{j}=C_{m}^{j}-\hat{\theta}_{j, 0}=C_{m}^{j}-s C_{m-2}^{j-1}+p C_{m-4}^{j-2},
$$

whence,

$$
\begin{gathered}
\pi_{j} \geq 0, \forall 0 \leq j \leq m \\
\Leftrightarrow \max _{0 \leq j \leq m} s(m-2)(m-3) j(m-j)-p j(j-1)(m-j)(m-j-1) \\
\leq m(m-1)(m-2)(m-3) .
\end{gathered}
$$

Therefore it is natural to introduce the function:

$$
f_{m}: x \longmapsto s(m-2)(m-3) x(m-x)-p x(x-1)(m-x)(m-x-1) .
$$

Clearly $f_{m}(m-x)=f_{m}(x)$. Thus, for $x \geq 0$, consider the more convenient

$$
\begin{aligned}
g_{m}(x)= & f_{m}\left(\frac{m}{2}+\sqrt{x}\right) \\
= & s(m-2)(m-3)\left(\frac{m^{2}}{4}-x\right)-p\left(\frac{m^{2}}{4}-x\right)\left(\frac{(m-2)^{2}}{4}-x\right) \\
= & \underbrace{\left[\frac{s}{4} m^{2}(m-2)(m-3)-\frac{p}{16} m^{2}(m-2)^{2}\right]}_{a_{m}} \\
& \quad-\underbrace{\left[s(m-2)(m-3)-\frac{p}{2}\left(m^{2}-2 m+2\right)\right]}_{b_{m}} x-p x^{2} .
\end{aligned}
$$

The following elementary lemma, which we state without proof, will now be helpful:

Lemma 3.2 Under condition (ii) of Proposition 3.5, we have:

$$
s^{2} \geq 4 p, s>\frac{p}{2} \quad \text { and } 0<\frac{s}{4}-\frac{p}{16}<1 .
$$

Hence, for $m$ sufficiently large, $a_{m}$ and $b_{m}$ are nonnegative. Then, the fact that $g_{m}\left(m^{2} / 4\right)=0$ and some other straightforward considerations lead to:

$$
\max _{0 \leq x \leq \frac{m^{2}}{4}} g_{m}(x)=g_{m}(0)=f_{m}\left(\frac{m}{2}\right)=\max _{0 \leq x \leq m} f_{m}(x) .
$$

Observing finally that

$$
g_{m}(0)=a_{m}=\frac{s}{4} m^{2}(m-2)(m-3)-\frac{p}{16} m^{2}(m-2)^{2}
$$

and remembering that $\frac{s}{4}-\frac{p}{16}<1$, we conclude that it is always possible to choose $m$ sufficiently large for (3.49) to be satisfied. 
Remark 3.5 (i) Proposition 3.5 allows us to give the complete list of generating polynomials of degree 2 . There are 46 solutions, corresponding to the following pairs $(s, p)$ :

$$
\begin{aligned}
& s=0 \Rightarrow-15 \leq p \leq-1 \\
& s=1 \Rightarrow-11 \leq p \leq-1 \\
& s=2 \Rightarrow-7 \leq p \leq 1 \text { (and } p \neq 0) \\
& s=3 \Rightarrow-3 \leq p \leq 2(\text { and } p \neq 0) \\
& s=4 \Rightarrow 1 \leq p \leq 4 \\
& s=4 \Rightarrow p=5 \text { or } p=6 \\
& s=5 \Rightarrow p=9
\end{aligned}
$$

(ii) Of course, these generating polynomials do not all provide a solution when $m=5$. In that case, a close look at the previous proof shows that condition (3.48), when $p>0$, is equivalent to $\beta \leq 3$. One checks that this is true for each pair $(s, p)$ listed above. Since the $\hat{\theta}_{j, k}$ are trivially $\geq 0$ when $p<0$, it suffices to discard the generating polynomials for which some of the

$$
\pi_{j}=C_{5}^{j}-s C_{3}^{j-1}+p C_{1}^{j-2}, \quad 0 \leq j \leq 5
$$

are negative. This happens for exactly 16 among the above pairs $(s, p)$.

Corollary 3.1 There are exactly $30+3+1=34$ stopping distributions for $m=5$.

The corresponding coefficients $\left(\pi_{j}, 0 \leq j \leq 5\right)$ are given below. Since $\pi_{0}=1$ and $\pi_{j}=\pi_{5-j}$, it is enough to specify $\pi_{1}$ and $\pi_{2}$ :

$$
\begin{array}{ll}
\pi_{1}=5 \Rightarrow 0 \leq \pi_{2} \leq 10 & \pi_{1}=2 \Rightarrow 0 \leq \pi_{2} \leq 3 \\
\pi_{1}=4 \Rightarrow 0 \leq \pi_{2} \leq 7 & \pi_{1}=1 \Rightarrow 0 \leq \pi_{2} \leq 2 \\
\pi_{1}=3 \Rightarrow 0 \leq \pi_{2} \leq 5 & \pi_{1}=0 \Rightarrow 0 \leq \pi_{2} \leq 1
\end{array}
$$

We end this section with the following

Open problem Take a distribution $\mathcal{L}=\left\{\left(m-2 j, p_{j}\right), 0 \leq j \leq m\right\}$ satisfying $\left(N_{1}\right)$ to $\left(N_{3}\right)$. Denote by

$$
\hat{B}(z)=\sum_{l=0}^{\mu} \hat{b}_{l} z^{\mu-l}
$$

the generating polynomial of $\mathcal{L}$ and assume that the coefficients in the power series expansion of $1 / \hat{B}(1 / z)$ are all $\geq 0$. Is it then always possible to find an integer $n$ sufficiently large for $\mathcal{L}_{n}\left(\hat{b}_{0}, \hat{b}_{1}, \ldots, \hat{b}_{\mu}\right)$ to be a stopping distribution? 


\section{Results for a two-dimensional walk}

Let $\left(S_{n}^{\prime}, n \geq 0\right)$ and $\left(S_{n}^{\prime \prime}, n \geq 0\right)$ be two independent symmetric Bernoulli random walks, starting from 0 . We denote the corresponding filtrations by $\left(\mathcal{F}_{n}^{\prime}, n \geq 0\right)$ and $\left(\mathcal{F}_{n}^{\prime \prime}, n \geq 0\right)$ respectively; we assume that (1.1) holds for either of them and moreover, that $\mathcal{F}_{\infty}^{\prime} \Perp \mathcal{F}_{\infty}^{\prime \prime}$. The two-dimensional walk $\mathbf{S}=\left(\left(S_{n}^{\prime}, S_{n}^{\prime \prime}\right), n \geq 0\right)$ is adapted to the filtration $\left(\mathcal{F}_{n}=\mathcal{F}_{n}^{\prime} \vee \mathcal{F}_{n}^{\prime \prime}, n \geq 0\right)$. Note that if $T$ is a $\left(\mathcal{F}_{n}^{\prime}\right)$ - stopping time such that $T \Perp S_{T}^{\prime}$, then $S_{T}^{\prime} \Perp S_{T}^{\prime \prime}$. This leads us more generally to investigate the class of $\left(\mathcal{F}_{n}\right)$-stopping times $T$ such that $S_{T}^{\prime} \Perp S_{T}^{\prime \prime}$. The analogous problem in case of the two-dimensional Brownian motion has been treated in [7].

\subsection{Stopping times $T$ such that $S_{T}^{\prime}$ and $S_{T}^{\prime \prime}$ are independent}

Theorem 4.1 Assume the above notations. Let $T$ be a $\left(\mathcal{F}_{n}\right)$-stopping time such that

$T$ is $S^{\prime}$-standard and $S^{\prime \prime}$-standard, $S_{T}^{\prime}$ and $S_{T}^{\prime \prime}$ have finite exponential moments of any order, $S_{T}^{\prime}$ and $S_{T}^{\prime \prime}$ are independent.

Then $\mathcal{L}\left(S_{T}^{\prime}\right)=\mathcal{L}\left(S_{T}^{\prime \prime}\right)=\mathcal{B S}(N)$, for some integer $N$, where the symmetric binomial distribution $\mathcal{B S}(N)$ is defined by (1.15).

Remark 4.1 (i) Theorem 4.1 will be extended to more general walks in the appendix.

(ii) Theorem 1.2 is actually a corollary of Theorem 4.1. Indeed, let $T$ be a $\left(\mathcal{F}_{n}^{\prime}\right)$-stopping time such that $g_{T}(r)=\mathbb{E}\left(r^{T}\right)<\infty, \forall r \geq 0$ and $S_{T}^{\prime} \Perp T$. We claim that $S_{T}^{\prime} \Perp S_{T}^{\prime \prime}$ and that $S_{T}^{\prime}$ and $S_{T}^{\prime \prime}$ have finite exponential moments of any order. The first statement is trivial, whereas the second follows from Theorem 1.1, a) in the case of $S_{T}^{\prime}$. For $S_{T}^{\prime \prime}$, we write

$$
\begin{aligned}
\mathbb{E}\left(\exp \lambda S_{T}^{\prime \prime}\right) & =\sum_{n=0}^{+\infty} \mathbb{E}\left(\exp \lambda S_{n}^{\prime \prime}\right) \mathbb{P}(T=n) \\
& =\sum_{n=0}^{+\infty} \phi(\lambda)^{n} \mathbb{P}(T=n) \\
& =g_{T}(\phi(\lambda))<\infty .
\end{aligned}
$$

Hence, by Theorem 4.1, for some integer $N$, we have $\mathbb{E}\left(\exp z S_{T}^{\prime}\right)=(\operatorname{ch} z)^{N}$, that is, $T=N$ a.s. 
Proof of Theorem 4.1. Proceeding as in Theorem 1.1, one shows first that

$$
M_{n}(\lambda, \mu)=\frac{\exp \left(\lambda S_{n}^{\prime}+\mu S_{n}^{\prime \prime}\right)}{(\operatorname{ch} \lambda \operatorname{ch} \mu)^{n}}
$$

is a martingale. By (4.1) and (4.2), whenever $|\operatorname{ch} \lambda \operatorname{ch} \mu| \geq 1$, the stopped martingale $M_{n}^{T}(\lambda, \mu)$ is uniformly integrable. In that case, we have

$$
\mathbb{E}\left[\frac{\exp \left(\lambda S_{T}^{\prime}+\mu S_{T}^{\prime \prime}\right)}{(\operatorname{ch} \lambda \operatorname{ch} \mu)^{T}}\right]=1 .
$$

To determine the joint distribution of $\left(S_{T}^{\prime}, S_{T}^{\prime \prime}\right)$, it suffices to consider the case where

$$
\operatorname{ch} \lambda \operatorname{ch} \mu=1,
$$

which occurs for instance when

$$
\lambda \in \mathbb{R} \text { and } \mu=i \arccos (1 / \operatorname{ch} \lambda) .
$$

Thus, assuming (4.4) and applying (4.3), we have

$$
\mathbb{E}\left(\exp \left(\lambda S_{T}^{\prime}\right)\right) \mathbb{E}\left(\exp \left(\mu S_{T}^{\prime \prime}\right)\right)=1 .
$$

Notice that if $(\lambda, \mu)$ satisfies $(4.4)$, then so does $(-\lambda, \mu)$, whence the distribution of $S_{T}^{\prime}$ (and likewise that of $S_{T}^{\prime \prime}$ ) is symmetric. Using (4.5) and introducing the notation $\psi_{1}(z)=\mathbb{E}\left(\exp z S_{T}^{\prime}\right)$ and $\psi_{2}(z)=\mathbb{E}\left(\exp z S_{T}^{\prime \prime}\right)$, we may rewrite (4.6) as

$$
\psi_{1}(\lambda) \psi_{2}\left(i \arccos \left(\frac{1}{\operatorname{ch} \lambda}\right)\right)=1, \forall \lambda \in \mathbb{R} .
$$

Since $\lim _{\lambda \rightarrow \pm \infty} \psi_{1}(\lambda)=+\infty$, by passing to the limit in (4.7), we obtain that $\psi_{2}\left(i \frac{\pi}{2}\right)=0$. It follows from the symmetry and the $2 i \pi$-periodicity of $\psi_{2}$ that

$$
\mathcal{Z}_{\psi_{2}} \supset \mathcal{Z}:=\left\{i\left(\frac{\pi}{2}+k \pi\right): k \in \mathbb{Z}\right\} .
$$

To show that actually $\mathcal{Z}_{\psi_{2}}=\mathcal{Z}$, observe that

$$
\forall \mu \in \mathbb{C} \backslash \mathcal{Z}, \exists \lambda \in \mathbb{C} \backslash \mathcal{Z}: \operatorname{ch} \lambda \operatorname{ch} \mu=1 \text {. }
$$

Substituting any such pair $(\lambda, \mu)$ in $(4.6)$, we conclude that $\psi_{2}(\mu) \neq 0$, for every $\mu \in \mathbb{C} \backslash \mathcal{Z}$, whence our claim follows. Exchanging $\psi_{1}$ and $\psi_{2}$ leads finally to

$$
\mathcal{Z}_{\psi_{1}}=\mathcal{Z}_{\psi_{2}}=\mathcal{Z}
$$

Note that the zeros of $\psi_{2}$ (resp. $\psi_{1}$ ) have a common multiplicity, denoted by $N$ (resp. $M$ ) throughout. This follows again from the symmetry and the periodicity of the functions. 
In our next step, we will prove that $\psi_{1}$ is an entire function of order 1 . First of all, we observe that the power series expansion of $\psi_{2}$ at the point $i \pi / 2$ may be written as follows:

$$
\psi_{2}(z)=\sum_{n=N}^{+\infty} a_{n}\left(i \frac{\pi}{2}-z\right)^{n}, z \in \mathbb{C}, a_{N} \neq 0 .
$$

Hence,

$$
\psi_{2}(i \arccos (r))=\sum_{n=N}^{+\infty} a_{n}(i \arcsin (r))^{n},-1 \leq r \leq 1 .
$$

Using the power series expansion of arcsin at 0 , we obtain that

$$
\left|\psi_{2}\left(i \arccos \left(\frac{1}{\operatorname{ch} \lambda}\right)\right)\right| \geq \frac{c}{(\operatorname{ch} \lambda)^{N}},
$$

for some constant $c>0$ and $\lambda \in \mathbb{R}$ sufficiently large. Under this condition, (4.7) implies that $\left|\psi_{2}(\lambda)\right| \leq c^{-1}(\operatorname{ch} \lambda)^{N}$, whence $\psi_{2}$ is of order $\leq 1$. As the Laplace transform of a non-zero random variable, it must be of order 1 .

Now, an application of Hadamard's factorization theorem for entire functions of finite order yields

$$
\psi_{1}(z)=\prod_{k=0}^{+\infty}\left(1+\frac{z^{2}}{\pi^{2}\left(k+\frac{1}{2}\right)^{2}}\right)^{M}=(\operatorname{ch} z)^{M} .
$$

Thus, applying again (4.6) with $\operatorname{ch} \lambda \operatorname{ch} \mu=1$, we get

$$
\psi_{2}(\mu)=\frac{1}{\psi_{1}(\lambda)}=\left(\frac{1}{\operatorname{ch} \lambda}\right)^{M}=(\operatorname{ch} \mu)^{M},
$$

whence $M=N$, that is, $S_{T}^{\prime}$ and $S_{T}^{\prime \prime}$ are both distributed according to $\mathcal{B S}(N)$.

\subsection{Stopping times such that $\mathbf{S}_{T} \sim \mathrm{S}_{n}$}

Of course, constant stopping times satisfy the assumptions of Theorem 4.1. Thus, we naturally wonder whether there are other standard stopping times $T$ such that, for fixed $n \in \mathbb{N}$,

$$
\mathbf{S}_{T} \sim \mathbf{S}_{n}
$$

Let us first investigate the analogous problem in the one-dimensional setting. More precisely, given a symmetric Bernoulli random walk $S$ on $\mathbb{Z}$, we ask for a standard, non constant stopping time $T$, such that, for fixed $n \in \mathbb{N}$,

$$
S_{T} \sim S_{n}
$$


Direct and straightforward calculations show that this problem has no solution for $n<4$. When $n \geq 4$, we will actually provide two solutions, the first being unbounded and the second bounded. Both stopping times will be constructed in the natural filtration of the walk.

Remark 4.2 It suffices to figure out a solution $T$ in the case $n=4$. Then, for $n=4+p, p \geq 0$, the stopping time $T_{p}=T+p$ trivially works.

\subsubsection{An unbounded stopping time satisfying (4.9)}

Let us first recall the target distribution, that is, $\mathcal{L}\left(S_{4}\right)$ :

\begin{tabular}{|c|c|c|c|c|c|}
\hline$k$ & -4 & -2 & 0 & 2 & 4 \\
\hline $\mathbb{P}\left(S_{4}=k\right)$ & $\frac{1}{16}$ & $\frac{4}{16}$ & $\frac{6}{16}$ & $\frac{4}{16}$ & $\frac{1}{16}$ \\
\hline
\end{tabular}

Now, a stopping time $T$, such that (4.9) holds, may be defined as follows:

$$
T=\left\{\begin{array}{l}
2, \text { if } S_{2} \in\{-2,2\}, \\
\inf \left\{n>2,\left|S_{n}\right|=4 \text { or } S_{n}=0\right\}, \text { otherwise. }
\end{array}\right.
$$

The verification is straightforward and left to the reader.

\subsubsection{A bounded stopping time satisfying (4.9)}

Here we describe a more general construction, which works whenever $n=2 m$ is even and $C_{2 m}^{m}$ meets some divisibility condition to be specified. Given an integer $p$ such that $0<p<m$, let $\Gamma_{p}$ be an arbitrary set of paths leading from the origin to the point $(2 p, 0)$. We will assume throughout that $\gamma_{p}:=\left|\Gamma_{p}\right|$ satisfies $0<\gamma_{p}<C_{2 p}^{p}$. Define a stopping time $T$ as follows:

- On $\Gamma_{p}$, set $T=2 p$ (so that $\left.S_{T}=0\right)$,

- On $\Gamma_{p}^{c} \cap\left\{S_{2 m} \neq 0\right\}$, set $T=2 m$,

- On $\Gamma_{p}^{c} \cap\left\{S_{2 m}=0\right\}$, set $T=4 m-2 p$.

The intuition behind this definition is to replace the contribution of the paths stopped at time $2 p$ by that of the paths which are only stopped at time $4 m-2 p$.

Before calculating the distribution of $S_{T}$, let us recall that the number of paths leading from the origin to $(2 m, 2 x)$ is given by $\lambda_{2 m, 2 x}:=C_{2 m}^{m+x}$. Now, for $x \neq 0$, we have

$\mathbb{P}\left(S_{T}=2 x\right)=\mathbb{P}\left(\Gamma_{p}^{c} \cap\left\{S_{2 m}=2 x\right\}\right)+\mathbb{P}\left(\Gamma_{p}^{c} \cap\left\{S_{2 m}=0\right\} \cap\left\{S_{4 m-2 p}=2 x\right\}\right)$. 
The first term is calculated as follows:

$$
\begin{aligned}
\mathbb{P}\left(\Gamma_{p}^{c} \cap\left\{S_{2 m}=2 x\right\}\right) & =\mathbb{P}\left(S_{2 m}=2 x\right)-\mathbb{P}\left(\Gamma_{p} \cap\left\{S_{2 m}=2 x\right\}\right) \\
& =\frac{\lambda_{2 m, 2 x}}{2^{2 m}}-\frac{\gamma_{p}}{2^{2 p}} \cdot \frac{\lambda_{2 m-2 p, 2 x}}{2^{2 m-2 p}}=\frac{\lambda_{2 m, 2 x}}{2^{2 m}}-\frac{\gamma_{p} \lambda_{2 m-2 p, 2 x}}{2^{2 m}} .
\end{aligned}
$$

Similarly, we get

$$
\mathbb{P}\left(\Gamma_{p}^{c} \cap\left\{S_{2 m}=0\right\} \cap\left\{S_{4 m-2 p}=2 x\right\}\right)=\frac{\lambda_{2 m-2 p, 2 x}}{2^{4 m-2 p}}\left(C_{2 m}^{m}-\gamma_{p} C_{2 m-2 p}^{m-p}\right) .
$$

Hence,

$$
\mathbb{P}\left(S_{T}=2 x\right)=\frac{\lambda_{2 m, 2 x}}{2^{2 m}}+\frac{\lambda_{2 m-2 p, 2 x}}{2^{4 m-2 p}}\left[C_{2 m}^{m}-\gamma_{p}\left(C_{2 m-2 p}^{m-p}+2^{2 m-2 p}\right)\right] .
$$

It remains to compute

$$
\begin{aligned}
\mathbb{P}\left(S_{T}=0\right) & =\mathbb{P}\left(\Gamma_{p}\right)+\mathbb{P}\left(\Gamma_{p}^{c} \cap\left\{S_{2 m}=0\right\} \cap\left\{S_{4 m-2 p}=0\right\}\right) \\
& =\frac{\gamma_{p}}{2^{2 p}}+\frac{C_{2 m-2 p}^{m-p}}{2^{4 m-2 p}}\left(C_{2 m}^{m}-\gamma_{p} C_{2 m-2 p}^{m-p}\right)
\end{aligned}
$$

where the last equality comes from substituting $x=0$ in (4.12).

Proposition 4.1 Let $T$ be the stopping time defined by (4.11). Then $S_{T} \sim$ $S_{2 m}$ if and only if

$$
\gamma_{p}\left(C_{2 m-2 p}^{m-p}+2^{2 m-2 p}\right)=C_{2 m}^{m} .
$$

Proof. (4.13) and (4.14) provide two necessary conditions, namely (4.15) and

$$
\frac{\gamma_{p}}{2^{2 p}}+\frac{C_{2 m-2 p}^{m-p}}{2^{4 m-2 p}}\left(C_{2 m}^{m}-\gamma_{p} C_{2 m-2 p}^{m-p}\right)=\frac{C_{2 m}^{m}}{2^{2 m}}
$$

It is easy to check that these two conditions are actually equivalent. By the definition of $T$, we must have moreover $\gamma_{p}<C_{2 p}^{p}$. But this is also implied by (4.15). Indeed, if $\gamma_{p} \geq C_{2 p}^{p}$, then we would have

$$
\begin{aligned}
\gamma_{p}\left(C_{2 m-2 p}^{m-p}+2^{2 m-2 p}\right) & \geq C_{2 p}^{p} C_{2 m-2 p}^{m-p}+C_{2 p}^{p} \sum_{k=0}^{2 m-2 p} C_{2 m-2 p}^{k} \\
& \geq C_{2 p}^{p} C_{2 m-2 p}^{m-p}+\sum_{k=0}^{2 m-2 p} C_{2 p}^{m-k} C_{2 m-2 p}^{k} \\
& =C_{2 p}^{p} C_{2 m-2 p}^{m-p}+C_{2 m}^{m}>C_{2 m}^{m}
\end{aligned}
$$

a contradiction. 
Setting $\lambda_{p}=C_{2 p}^{p}$ and assuming that $p$ and $\gamma_{p}$ satisfy (4.15), there are exactly $C_{\lambda_{p}}^{\gamma_{p}}$ stopping times $T$ of the type (4.11) for which $S_{T} \sim S_{2 m}$. (This comes from the fact that the definition of $T$ does not depend on the choice of the elements in $\Gamma_{p}$, but only on their number.) Moreover, note that these stopping times have the same distribution.

Thus, it is natural to look for pairs $\left(p, \gamma_{p}\right)$ such that $0<p<m$ and condition (4.15) is satisfied. Setting $p=m-1$ (resp. $p=m-2$ ), this condition reads $6 \gamma_{m-1}=C_{2 m}^{m}$ (resp. $22 \gamma_{m-2}=C_{2 m}^{m}$ ). Note in particular that one may take $p=\gamma_{p}=1$, when $n=4$. More generally, since $C_{2 m}^{m}$ is always even and in view of Remark 3.1, we may state the following

Corollary 4.1 For every $n \geq 4$ there exists a bounded and non constant stopping time $T$ satisfying (4.9). If $n=2 m$ is even and $C_{2 m}^{m}$ is divisible by 3 (resp. 11), then there are at least $C_{\lambda_{p}}^{\gamma_{p}}$ different ways to construct this stopping time, with $p=m-1$ (resp. $p=m-2$ ).

Remark 4.3 The following divisibility criterion from elementary number theory is a particular case of Kummer's result (cf. [5]): Let $m$ be a positive integer and $p$ be a prime number. Then $p$ divides $C_{2 m}^{m}$ if and only if in the $p$-adic representation of $m$ appears at least one coefficient that is $\geq p / 2$.

Question Is it possible to count the stopping times T such that (4.9) holds?

\subsubsection{A stopping time satisfying (4.8) when $n=4$}

Let us come back to the 2-dimensional problem (4.8). In view of Remark 4.2, it is enough to give a solution when $n=4$. First of all, we recall that $\mathbf{S}_{4} \sim \mathcal{B S}(4) \otimes \mathcal{B S}(4)$. The support of this distribution is given in figure 2 below.

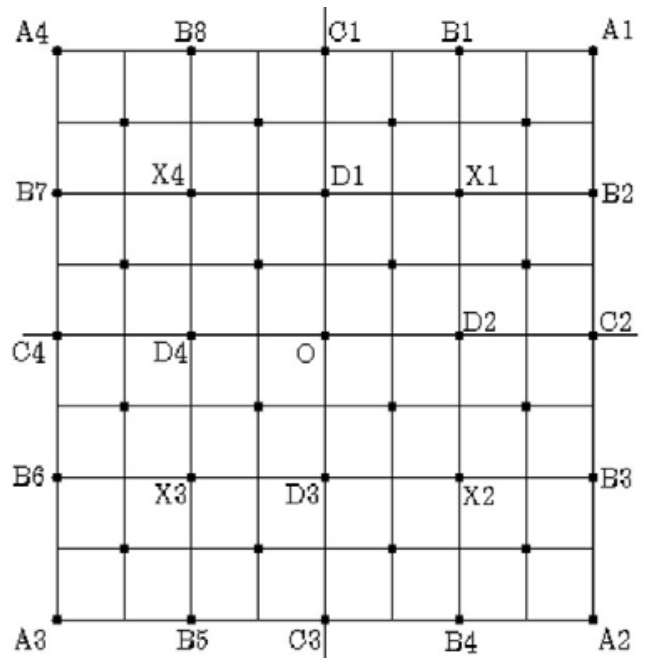

Fig. 2: Support of $\mathcal{L}\left(\mathbf{S}_{4}\right)$ 
Assuming the corresponding notation, we see that

- points $A_{1}$ to $A_{4}$ are reached with probability $\frac{1}{16^{2}}$,

- $\quad$ points $B_{1}$ to $B_{8}$ are reached with probability $\frac{4}{16^{2}}$,

- points $C_{1}$ to $C_{4}$ are reached with probability $\frac{6}{16^{2}}$,

- points $X_{1}$ to $X_{4}$ are reached with probability $\frac{16}{16^{2}}$,

- $\quad$ points $D_{1}$ to $D_{4}$ are reached with probability $\frac{24}{16^{2}}$,

- $\quad$ point $O$ is reached with probability $\frac{36}{16^{2}}$.

Let $\mathcal{X}=\left\{X_{1}, X_{2}, X_{3}, X_{4}\right\}$. We define a stopping time $T$ as follows:

- if $\mathbf{S}_{2} \in \mathcal{X}$ then $T=2$,

- if $\mathbf{S}_{2} \notin \mathcal{X}$ and $\mathbf{S}_{4} \notin \mathcal{X}$ then $T=4$,

- if $\mathbf{S}_{2} \notin \mathcal{X}$ and $\mathbf{S}_{4} \in \mathcal{X}$ then $T=\inf \left\{n>4 /\left\|\mathbf{S}_{n}-\mathbf{S}_{4}\right\|=2\right\}$.

Here and in all that follows, we use the notation $\|(x, y)\|=\max (|x|,|y|)$. In our computations, we will have to consider the walk started from other points than the origin: we will denote by $\mathbb{P}_{M}$ the conditional probability given that $\left\{\mathbf{S}_{0}=M\right\}$. We will still write $\mathbb{P}$ for $\mathbb{P}_{O}$. The determination of $\mathcal{L}\left(\mathbf{S}_{T}\right)$ requires some elementary lemmas, which we state without proof.

Lemma 4.1 Given that the walk starts from $O$, the distribution of $\mathbf{S}_{2}$ is:

$$
\mathbb{P}\left(\mathbf{S}_{2}=X_{i}\right)=\frac{1}{16} ; \mathbb{P}\left(\mathbf{S}_{2}=D_{i}\right)=\frac{2}{16} ; \mathbb{P}\left(\mathbf{S}_{2}=O\right)=\frac{4}{16}, i \in\{1,2,3,4\} .
$$

Lemma 4.2 Set $\theta=\inf \left\{n \geq 0,\left\|\mathbf{S}_{n}-\mathbf{S}_{0}\right\|=2\right\}$. Given that the walk starts from $O$, the distribution of $\mathbf{S}_{\theta}$ is:

$$
\mathbb{P}\left(\mathbf{S}_{\theta}=X_{i}\right)=\frac{1}{12} ; \mathbb{P}\left(\mathbf{S}_{\theta}=D_{i}\right)=\frac{2}{12}, i \in\{1,2,3,4\} .
$$

Lemma 4.3 The probability that the walk starting from $O$ reaches one of the points $X_{i}$ at time $n=4$, without having been stopped before, is given by

$$
\mathbb{P}\left(\mathbf{S}_{2} \notin \mathcal{X} \text { and } \mathbf{S}_{4}=X_{i}\right)=\frac{12}{16^{2}}, i \in\{1,2,3,4\} .
$$

Proposition 4.2 The stopping time $T$ defined by (4.17) is standard and $\mathbf{S}_{T} \sim \mathbf{S}_{4}$.

Proof. It is clear that $T$ is standard since $\left\|\mathbf{S}_{n \wedge T}\right\| \leq 4$, for every $n \geq 0$. To calculate the distribution of $\mathbf{S}_{T}$, we will constantly use Lemmas 4.1, 4.2 and 4.3. For instance,

$$
\begin{aligned}
\mathbb{P}\left(\mathbf{S}_{T}=A_{1}\right) & =\mathbb{P}\left(\{T>4\} \cap\left\{\mathbf{S}_{T}=A_{1}\right\}\right) \\
& =\mathbb{P}\left(\left\{\mathbf{S}_{2} \notin \mathcal{X}\right\} \cap\left\{\mathbf{S}_{4}=X_{1}\right\} \cap\left\{\mathbf{S}_{T}=A_{1}\right\}\right) \\
& =\mathbb{P}\left(\left\{\mathbf{S}_{2} \notin \mathcal{X}\right\} \cap\left\{\mathbf{S}_{4}=X_{1}\right\}\right) \mathbb{P}_{X_{1}}\left(\mathbf{S}_{\theta}=A_{1}\right) \\
& =\frac{12}{16^{2}} \cdot \frac{1}{12}=\frac{1}{16^{2}}
\end{aligned}
$$


and

$$
\begin{aligned}
\mathbb{P} & \left(\mathbf{S}_{T}=B_{1}\right)=\mathbb{P}\left(\{T=4\} \cap\left\{\mathbf{S}_{4}=B_{1}\right\}\right)+\mathbb{P}\left(\{T>4\} \cap\left\{\mathbf{S}_{T}=B_{1}\right\}\right) \\
& =\mathbb{P}\left(\left\{\mathbf{S}_{2}=D_{1}\right\} \cap\left\{\mathbf{S}_{4}=B_{1}\right\}\right)+\mathbb{P}\left(\left\{\mathbf{S}_{2} \notin \mathcal{X}\right\} \cap\left\{\mathbf{S}_{4}=X_{1}\right\} \cap\left\{\mathbf{S}_{T}=B_{1}\right\}\right) \\
& =\mathbb{P}\left(\mathbf{S}_{2}=D_{1}\right) \mathbb{P}_{D_{1}}\left(\mathbf{S}_{2}=B_{1}\right)+\mathbb{P}\left(\left\{\mathbf{S}_{2} \notin \mathcal{X}\right\} \cap\left\{\mathbf{S}_{4}=X_{1}\right\}\right) \mathbb{P}_{X_{1}}\left(\mathbf{S}_{\theta}=B_{1}\right) \\
& =\frac{2}{16} \cdot \frac{1}{16}+\frac{12}{16^{2}} \cdot \frac{2}{12}=\frac{4}{16^{2}} .
\end{aligned}
$$

The remaining probabilities are obtained by similar calculations. The reader may easily verify that they agree with $\mathcal{B S}(4) \otimes \mathcal{B S}(4)$, hence completing the proof.

Remark 4.4 Likewise, it is possible to construct a bounded non constant stopping time $T$ such that $\mathbf{S}_{T} \sim \mathcal{B S}(4) \otimes \mathcal{B S}(4)$, by taking the corresponding construction in dimension 1 as a source of inspiration.

\section{Appendix : Generalization of Theorem 4.1}

Here we use again the notation of Section 4 , but the common increment $X$ of the random walks $\left(S_{n}^{\prime}, n \geq 0\right)$ and $\left(S_{n}^{\prime \prime}, n \geq 0\right)$ will be more general, namely:

(A) $X$ is integer-valued, symmetric and not constant;

(B) $\varphi(z)=\mathbb{E}(\exp z X)$ is entire, of order 1 , and with zeros that are all simple and located in the vertical strip $[-a, a] \times \mathbb{R}$, where $a>0$.

Theorem 5.1 Under the above assumptions, let $T$ be a $\left(\mathcal{F}_{n}\right)$-stopping time such that:

$$
\begin{aligned}
& T \text { is } S^{\prime} \text {-standard and } S^{\prime \prime} \text {-standard, } \\
& S_{T}^{\prime} \text { and } S_{T}^{\prime \prime} \text { have finite exponential moments of any order, } \\
& S_{T}^{\prime} \text { and } S_{T}^{\prime \prime} \text { are independent. }
\end{aligned}
$$

Then, for some integer $N$, we have $S_{T}^{\prime} \sim S_{T}^{\prime \prime} \sim S_{N}$, that is,

$$
\mathbb{E}\left(\exp z S_{T}^{\prime}\right)=\mathbb{E}\left(\exp z S_{T}^{\prime \prime}\right)=\varphi(z)^{N} .
$$

Proof. We need a few preliminary lemmas:

Lemma 5.1 Assuming that conditions (A) and (B) are satisfied, we have i) $\varphi(\mathbb{C})=\mathbb{C}$, and

ii) $\lim _{|z| \rightarrow \infty}|\varphi(z)|=\infty$, in any horizontal strip $|\Im m z| \leq b$, where $b>0$. 
Proof of Lemma 5.1. i) Given a complex number $u$, we ask for a solution $z$ of the equation $\varphi(z)=u$. Write $\eta_{u}(z)$ for $\varphi(z)-u$ and note that this function is still entire, symmetric and of order 1 . If $\eta_{u}$ vanishes for some $z_{0} \in \mathbb{C}$, then $z_{0}$ is the required solution. Otherwise, by Hadamard's factorization theorem, we have $\eta_{u}(z)=\exp (c+d z)$, for some constants $c$ and $d$, whence $\varphi(z)=u+e^{c+d z}$. Since $\varphi$ is symmetric, $d$ must be 0 , so that $\varphi(z)=u+e^{c}$, which contradicts the fact that $\varphi$ is not constant.

ii) We denote by $\pm \alpha_{1}, \pm \alpha_{2}, \pm \alpha_{3}, \ldots$ the sequence of the zeros of $\varphi$, ordered in such a way that $\left(\left|\alpha_{n}\right|\right)_{n \geq 1}$ is increasing. By Hadamard's factorization theorem, we have the following representation formula:

$$
\varphi(z)=\prod_{k=1}^{\infty}\left(1-\frac{z^{2}}{\alpha_{k}^{2}}\right), \text { where } \sum_{k=1}^{\infty} \frac{1}{\left|\alpha_{k}\right|^{2}}<\infty .
$$

Since $\left|\alpha_{k}\right| \rightarrow+\infty$ and $\left(\Re e\left(\alpha_{k}\right)\right)_{k \geq 1}$ is bounded, we have:

$$
\begin{gathered}
\forall \nu>0, \exists K(\nu) \in \mathbb{N}, k>K(\nu) \Rightarrow \\
\arg \left(\alpha_{k}\right) \in[\pi / 2-\nu, \pi / 2+\nu] \cup[3 \pi / 2-\nu, 3 \pi / 2+\nu] \quad(\bmod 2 \pi) .
\end{gathered}
$$

Now, let $\left(z_{n}\right)_{n \geq 0}$ be a sequence of complex numbers such that $|\Im m z| \leq b$ and $\left|z_{n}\right| \rightarrow+\infty$. Consequently,

$$
\forall \varepsilon>0, \exists N(\varepsilon) \in \mathbb{N}, n>N(\varepsilon) \Rightarrow-\varepsilon \leq \arg \left(z_{n}^{2}\right) \leq \varepsilon .
$$

As a result, for $k \geq K(\nu)$ and $n \geq N(\epsilon)$,

$$
\pi-\varepsilon-2 \nu \leq \arg \left(\frac{z_{n}^{2}}{\alpha_{k}^{2}}\right) \leq \pi+\varepsilon+2 \nu .
$$

Choosing $\varepsilon$ and $\nu$ sufficiently small, we have, for $k \geq K(\nu)$ and $n \geq N(\varepsilon)$,

$$
\left.\arg \left(\frac{z_{n}^{2}}{\alpha_{k}^{2}}\right) \in\right] \frac{\pi}{2}, \frac{3 \pi}{2}[
$$

so that,

$$
\left|1-\frac{z_{n}^{2}}{\alpha_{k}^{2}}\right| \geq 1 \text { and consequently }\left|\varphi\left(z_{n}\right)\right| \geq \prod_{k=0}^{K(\nu)}\left|1-\frac{z_{n}^{2}}{\alpha_{k}^{2}}\right| .
$$

Thus, $\lim _{n \rightarrow \infty}\left|\varphi\left(z_{n}\right)\right|=+\infty$.

Now, recall the notation used in Section 4: $\psi_{1}(z)=\mathbb{E}\left(\exp z S_{T}^{\prime}\right)$ and $\psi_{2}(z)=\mathbb{E}\left(\exp z S_{T}^{\prime \prime}\right)$. We write still $\mathcal{Z}_{\varphi}$ (resp. $\left.\mathcal{Z}_{\psi_{1}}, \mathcal{Z}_{\psi_{2}}\right)$ for the set of the zeros of $\varphi$ (resp. $\psi_{1}, \psi_{2}$ ). As in Theorem 4.1, it is easily seen that, for every complex numbers $\lambda$ and $\mu$,

$$
\varphi(\lambda) \varphi(\mu)=1 \quad \Rightarrow \quad \psi_{1}(\lambda) \psi_{2}(\mu)=1 .
$$

This shows in particular that $\psi_{1}$ and $\psi_{2}$ are even. 
Lemma 5.2 Under the assumptions $(A)$ and $(B)$, we have $\mathcal{Z}_{\varphi}=\mathcal{Z}_{\psi_{1}}=\mathcal{Z}_{\psi_{2}}$.

Proof of Lemma 5.2. Since $\psi_{1}$ and $\psi_{2}$ play symmetric roles, it suffices to prove that $\mathcal{Z}_{\varphi}=\mathcal{Z}_{\psi_{1}}$, for instance. To show that $\mathcal{Z}_{\varphi} \subseteq \mathcal{Z}_{\psi_{1}}$, let $\alpha$ be a zero of $\varphi$. Choose a sequence of complex numbers $\left(\lambda_{n}\right)_{n>1}$ converging to $\alpha$, such that $\varphi\left(\lambda_{n}\right) \in \mathbb{R}_{+}^{*}$. Clearly, since $\varphi\left(\lambda_{n}\right)$ converges to 0 , there exists a sequence of real numbers $\left(\mu_{n}\right)_{n \geq 1}$ tending to $+\infty$, such that $\varphi\left(\lambda_{n}\right) \varphi\left(\mu_{n}\right)=1$, for every $n \geq 1$. Thus, (5.4) implies that $\psi_{1}\left(\lambda_{n}\right) \psi_{2}\left(\mu_{n}\right)=1$. Since $\psi_{2}\left(\mu_{n}\right) \rightarrow+\infty$, we have $\psi_{1}\left(\lambda_{n}\right) \rightarrow 0$, whence $\psi_{1}(\alpha)=0$, the desired result.

For the converse, let $\alpha^{\prime}$ be a zero of $\psi_{1}$. Choose a sequence of complex numbers $\left(\lambda_{n}\right)_{n \geq 1}$ converging to $\alpha^{\prime}$, such that $\psi_{1}\left(\lambda_{n}\right) \in \mathbb{R}_{+}^{*}$ and $\varphi\left(\lambda_{n}\right) \neq 0$. By the first statement of Lemma 5.1, there exists of a sequence of complex numbers $\left(\mu_{n}\right)_{n \geq 1}$, such that $\varphi\left(\lambda_{n}\right) \varphi\left(\mu_{n}\right)=1$. Remembering that $\varphi$ is periodic, we may assume moreover that $\left(\left|\Im m \mu_{n}\right|\right)_{n \geq 1}$ is bounded. Applying once more (5.4) yields $\psi_{1}\left(\lambda_{n}\right) \psi_{2}\left(\mu_{n}\right)=1$. From this we deduce that $\psi_{2}\left(\mu_{n}\right) \rightarrow+\infty$, which in turn shows that $\left|\Re e\left(\mu_{n}\right)\right| \rightarrow+\infty$. Now, by the second statement of Lemma 1 , we have $\left|\varphi\left(\mu_{n}\right)\right| \rightarrow+\infty$, whence $\varphi\left(\lambda_{n}\right) \rightarrow 0$. Hence, by continuity, $\varphi\left(\alpha^{\prime}\right)=0$.

Lemma 5.3 Under assumptions $(A)$ and $(B), \psi_{1}$ and $\psi_{2}$ are of order 1.

Proof of Lemma 5.3. Let $\alpha$ be a zero of $\varphi$. Recall that the zeros of $\varphi$ are all simple, by hypothesis. Hence, by the local inversion theorem for holomorphic functions, there exist neighbourhoods $U$ and $V$ of $\alpha$ and 0 respectively, such that the restriction $\varphi: U \rightarrow V$ is one-to-one. Its local inverse will be denoted by $\varphi^{-1}$ throughout.

Now, pick $\varepsilon>0$, such that $\left[0, \varepsilon\left[\times\{0\} \subset V\right.\right.$. Since $\lim _{\lambda \rightarrow \infty} \varphi(\lambda)=+\infty$, there exists a real number $\Lambda>0$, such that $\lambda \geq \Lambda \Rightarrow \varphi(\lambda)>1 / \varepsilon$. The local inverse $\varphi^{-1}$ being holomorphic on $V$, we have

$$
\varphi^{-1}(z)=\alpha+\beta z+o(z), z \in V
$$

for some $\beta \neq 0$. Thus,

$$
\varphi^{-1}\left(\frac{1}{\varphi(\lambda)}\right)=\alpha+\beta \frac{1}{\varphi(\lambda)}+o\left(\frac{1}{\varphi(\lambda)}\right), \lambda \geq \Lambda
$$

By Lemma 5.2, $\psi_{2}(\alpha)=0$, so that, in a neigbourhood of $\alpha$,

$$
\psi_{2}(z)=\gamma(z-\alpha)^{n}+o\left((z-\alpha)^{n}\right),
$$

for some integer $n \geq 1$ and $\gamma \neq 0$. In particular, for $\lambda \geq \Lambda$,

$$
\psi_{2}\left(\varphi^{-1}\left(\frac{1}{\varphi(\lambda)}\right)\right)=\gamma \beta^{n}\left(\frac{1}{\varphi(\lambda)}\right)^{n}+o\left(\left(\frac{1}{\varphi(\lambda)}\right)^{n}\right) .
$$


Since

$$
\varphi(\lambda) \varphi\left(\varphi^{-1}\left(\frac{1}{\varphi(\lambda)}\right)\right)=1
$$

we have by (5.4):

$$
\psi_{1}(\lambda) \psi_{2}\left(\varphi^{-1}\left(\frac{1}{\varphi(\lambda)}\right)\right)=1
$$

Using (5.5), we obtain that

$$
\psi_{1}(\lambda)=\frac{1+o(1)}{\gamma \beta^{n}\left(\frac{1}{\varphi(\lambda)}\right)^{n}},
$$

whence, $\left|\psi_{1}(\lambda)\right| \leq C(\varphi(\lambda))^{n}$, for some constant $C$ and $\lambda$ sufficiently large. This inequality shows that the order of $\psi_{1}$ is 1 and similar arguments yield the same result for $\psi_{2}$.

We come to the final step in our proof. Even if it means changing indices, we denote from now on by $\alpha_{1}, \ldots, \alpha_{k}$ the zeros (simple and distinct) of $\varphi$ such that $-\pi / 2<\arg \left(\alpha_{j}\right) \leq \pi / 2$ and $-\pi<\Im m \alpha_{j} \leq \pi, 1 \leq j \leq k$, so that $\mathcal{Z}_{\varphi}=\left\{ \pm \alpha_{j}+m \cdot 2 i \pi, 1 \leq j \leq k, m \in \mathbb{Z}\right\}$, by assumptions (A) and (B). Applying once more Hadamard's factorization theorem, we have

$$
\varphi=\xi_{1} \cdot \ldots \cdot \xi_{k} \text { where } \xi_{j}(z)=\prod_{m=-\infty}^{+\infty}\left(1-\frac{z^{2}}{\left(\alpha_{j}+m \cdot 2 i \pi\right)^{2}}\right) .
$$

The $\xi_{j}$ are $2 i \pi$-periodic. Their zeros are simple and moreover,

$$
\forall i, j \in\{1,2, \ldots, k\}, \quad \xi_{j}\left(\alpha_{j}\right)=0 \text { and } \xi_{j}\left(\alpha_{i}\right) \neq 0 \text { if } i \neq j .
$$

Thus, every $\alpha_{j}$ has a neighbourhood $V_{\alpha_{j}}$, such that the restriction of $\varphi$ to this neighbourhood, denoted by $\varphi_{j}$, is one-to-one. We write $\varphi_{j}^{-1}$ for its local inverse. By using Lemmas 5.2 and 5.3 as well as the symmetry of $\varphi_{1}$ and $\varphi_{2}$, it is easily seen that

$$
\psi_{1}=\xi_{1}^{n_{1}} \cdot \ldots \cdot \xi_{k}^{n_{k}} \text { and } \psi_{2}=\xi_{1}^{m_{1}} \cdot \ldots \cdot \xi_{k}^{m_{k}},
$$

for some positive integers $n_{1}, n_{2}, \ldots, n_{k}$ and $m_{1}, m_{2}, \ldots, m_{k}$. Without loss of generality, we may assume that $m_{1}$ is the minimum of these exponents. Now, since

$$
\varphi(\lambda) \cdot \varphi\left(\varphi_{1}^{-1}\left(\frac{1}{\varphi(\lambda)}\right)\right)=1,
$$

for real and sufficiently large $\lambda$ 's, we have by (5.4):

$$
\psi_{1}(\lambda) \psi_{2}\left(\varphi_{1}^{-1}\left(\frac{1}{\varphi(\lambda)}\right)\right)=1,
$$


or equivalently, using (5.7),

$$
\left(\xi_{1}^{n_{1}} \cdot \ldots \cdot \xi_{k}^{n_{k}}\right)(\lambda) \cdot\left(\xi_{1}^{m_{1}} \cdot \ldots \cdot \xi_{k}^{m_{k}}\right)\left(\varphi_{1}^{-1}\left(\frac{1}{\varphi(\lambda)}\right)\right)=1
$$

But, for sufficiently large $\lambda$,

$$
\varphi^{m_{1}}(\lambda) \varphi^{m_{1}}\left(\varphi_{1}^{-1}\left(\frac{1}{\varphi(\lambda)}\right)\right)=1
$$

so that (5.8) simplifies to

$$
\left(\xi_{1}^{n_{1}-m_{1}} \cdot \ldots \cdot \xi_{k}^{n_{k}-m_{1}}\right)(\lambda) \cdot\left(\xi_{2}^{m_{2}-m_{1}} \cdot \ldots \cdot \xi_{k}^{m_{k}-m_{1}}\right)\left(\varphi_{1}^{-1}\left(\frac{1}{\varphi(\lambda)}\right)\right)=1 .
$$

Letting $\lambda$ tend to $+\infty$ in (5.9), we get

$$
\lim _{\lambda \rightarrow+\infty}\left[\xi_{1}^{n_{1}-m_{1}}(\lambda) \cdot \ldots \cdot \xi_{k}^{n_{k}-m_{1}}(\lambda)\right] \cdot \xi_{2}^{m_{2}-m_{1}}\left(\alpha_{1}\right) \cdot \ldots \cdot \xi_{k}^{m_{k}-m_{1}}\left(\alpha_{1}\right)=1
$$

Since $\lim _{\lambda \rightarrow+\infty}\left|\xi_{j}(\lambda)\right|=+\infty$, for every $1 \leq j \leq k$, we deduce from (5.6) that $n_{1}=n_{2}=\cdots=n_{k}=m_{1}$. Hence $\psi_{1}=\varphi^{m_{1}}$. Substituting this in (5.4) leads without difficulty to $\psi_{2}=\varphi^{m_{1}}$, whence $\psi_{2}=\psi_{1}$.

\section{Acknowledgments}

We are indebted to Pierre Vallois for a first draft of Example 4. We also wish to thank Marc Yor for many valuable suggestions and for a first reading of the paper during the 2002 Saint-Flour Summer School. In particular, the simple proof of Lemma 1.4 is due to him.

\section{References}

[1] Bons, R. Pн.: Entire Functions. Academic Press Inc., New York, 1954.

[2] Bourekh, Y.: Quelques résultats sur le problème de Skorokhod. PhD thesis, Université de Paris VI, 1996.

[3] Falkner, N. and Fitzsimmons, P. J.: Stopping distributions of right processes. Probab. Theory Related Fields 89 (1991), 301-318.

[4] Feller, W.: An Introduction to Probability Theory and Its Applications, Vol. I. Third Edition. John Wiley \& Sons, Inc., New York, 1968.

[5] Kummer, E. E.: Über Ergänzungssätze zu den allgemeinen Reziprozitätsgesetzen. Journal für die reine und angewandte Mathematik [J. Reine Angew. Math.] 44 (1852), 93-146.

[6] LucAcs, E.: Characteristic Functions, $2^{\text {nd }}$ edition. Griffin, 1970. 
[7] De Meyer, B., Roynette, B., Vallois, P. And Yor, M.: On independent times and positions for Brownian motion. Rev. Mat. Iberoamericana 18 (2002), no. 3, p. 541-586.

[8] De Meyer, B., Roynette, B., Vallois, P. and Yor, M.: Sur l'indépendance d'un temps d'arrêt $T$ et de la position $B_{T}$ d'un mouvement brownien $\left(B_{u}, u \geq 0\right)$. C. R. Acad. Sci. Paris Sér. I Math. 333 (2001), 1017-1022.

[9] Newman, C. M.: An extension of Khinchine's inequality. Bull. Amer. Math. Soc. 81 (1975), no. 5, 913-915.

[10] Pitman, J. W.: One-dimensional Brownian motion and the threedimensional Bessel process. Advances in Appl. Probability 7 (1975), 511526.

[11] Rost, H.: The stopping distributions of a Markov process. Invent. Math. 14 (1971), 1-16.

[12] Roynette, B., Vallois, P. And Yor, M.: A solution to Skorokhod's embedding for linear Brownian motion and its local time. Studia Sci. Math. Hungar. 39 (2002), 97-127.

[13] Valiron, G.: Théorie des fonctions, $2^{\text {me }}$ édition. Masson, Paris, 1955.

Recibido: 22 de octubre de 2002

Christophe Ackermann

Département de Mathématiques

Institut Elie Cartan, BP239

54506 Vandœuvre-lès-Nancy Cedex, France

ackerman@iecn.u-nancy.fr

Gérard Lorang

Centre Universitaire de Luxembourg 162A, avenue de la Faïencerie

L-1511 Luxembourg

lorang@cu.lu

Bernard Roynette

Département de Mathématiques

Institut Elie Cartan, BP239

54506 Vandouvre-lès-Nancy Cedex, France bernard.roynette@iecn.u-nancy.fr 